\title{
Summary of overlap $\{\eta\}$
}

Information is compiled on three different levels:

(i) Overlap of nodes within a family $\left(\eta_{i}, \eta_{i}\right)$

(ii) Overlap among pair of nodes of two different families $\left(\eta_{i}, \eta_{j}\right)$

(iii) Overlap involving $n$ nodes, $n \geq 3$

It has emerged over a certain time span and consequently notation is not strictly uniform.

Symbols $\mathrm{h}, \mathrm{I}, \mathrm{m}, \mathrm{n}$ and $\mathrm{p}$ represent integers (positive or negative)

(i) : $\left(\eta_{i}, \eta_{i}\right)$

$\left(\eta_{1}, \eta_{1}\right)$

Special:

\begin{tabular}{|c|c|c|c|}
\hline \multicolumn{4}{|c|}{$(h \odot \Theta)_{i} \equiv(\hbar \odot)_{j}$} \\
\hline$i$ & $\eta_{1 \mathrm{~A}}$ & $\eta_{1 \mathrm{C}}$ & $\eta_{1 \mathrm{E}}$ \\
\hline$j$ & $\eta_{1 \mathrm{~B}}$ & $\eta_{1 \mathrm{D}}$ & $\eta_{1 \mathrm{~F}}$ \\
\hline
\end{tabular}

$\left(\eta_{2}, \eta_{2}\right)$

Special:

\begin{tabular}{|c|c|c|}
\hline \multicolumn{2}{|c|}{$(h \bar{h})_{\mathrm{i}} \equiv\left(\bar{h} \boldsymbol{h} \boldsymbol{O}_{\mathrm{j}}\right.$} \\
\hline $\mathrm{i}$ & $\eta_{2 \mathrm{~A}}$ & $\eta_{2 \mathrm{C}}$ \\
\hline$j$ & $\eta_{2 \mathrm{~B}}$ & $\eta_{2 \mathrm{D}}$ \\
\hline
\end{tabular}

$\left(h \odot \Theta^{\prime}\right)_{i} \equiv(\Theta h \odot)_{j}$

\begin{tabular}{|c|c|c|}
\hline$i$ & $\eta_{2 \mathrm{~A}}$ & $\eta_{2 \mathrm{~B}}$ \\
\hline$j$ & $\eta_{2 \mathrm{C}}$ & $\eta_{2 \mathrm{D}}$ \\
\hline
\end{tabular}

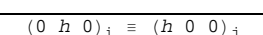

\begin{tabular}{|c|c|c|}
\hline$i$ & $\eta_{2 \mathrm{~A}}$ & $\eta_{2 \mathrm{~B}}$ \\
\hline$j$ & $\eta_{2 \mathrm{D}}$ & $\eta_{2 \mathrm{C}}$ \\
\hline
\end{tabular}

$\left(\eta_{3}, \eta_{3}\right)$

Special:

\begin{tabular}{|c|c|c|}
\hline \multicolumn{2}{|c|}{$(2 h \hbar)_{i} \equiv(2 h h)_{j}$} \\
\hline$i$ & $\eta_{3 \mathrm{~A}}$ & $\eta_{3 \mathrm{C}}$ \\
\hline$j$ & $\eta_{3 \mathrm{~B}}$ & $\eta_{3 \mathrm{D}}$ \\
\hline
\end{tabular}

$(h h \odot)_{i} \equiv(h 2 h \odot)_{j}$

\begin{tabular}{|l|l|l|}
\hline$i$ & $\eta_{3 \mathrm{~A}}$ & $\eta_{3 \mathrm{~B}}$ \\
\hline$j$ & $\eta_{3 \mathrm{C}}$ & $\eta_{3 \mathrm{D}}$ \\
\hline
\end{tabular}

$(h \overline{2 \hbar} \odot)_{i} \equiv(\bar{h} \odot)_{j}$

\begin{tabular}{|c|c|c|}
\hline$i$ & $\eta_{3 \mathrm{~A}}$ & $\eta_{3 \mathrm{~B}}$ \\
\hline$j$ & $\eta_{3 \mathrm{D}}$ & $\eta_{3 \mathrm{C}}$ \\
\hline
\end{tabular}

$\left(\eta_{4}, \eta_{4}\right)$

Special:

\begin{tabular}{|c|c|c|c|c|c|c|}
\hline \multicolumn{7}{|c|}{$(\odot \odot \odot)_{i} \equiv(\odot \odot \odot I)_{j}$} \\
\hline i & $\eta_{4 \mathrm{~A}}$ & $\eta_{4 \mathrm{C}}$ & $\eta_{4 \mathrm{E}}$ & $\eta_{4 \mathrm{G}}$ & $\eta_{4 \mathrm{I}}$ & $\eta_{4 \mathrm{~K}}$ \\
\hline j & $\eta_{4 \mathrm{~B}}$ & $\eta_{4 \mathrm{D}}$ & $\eta_{4 \mathrm{~F}}$ & $\eta_{4 \mathrm{H}}$ & $\eta_{4 \mathrm{~J}}$ & $\eta_{4 \mathrm{~L}}$ \\
\hline
\end{tabular}

\begin{tabular}{|c|c|c|c|c|}
\hline \multicolumn{4}{|c|}{$(h \overline{2 h} \odot)_{\mathrm{i}} \equiv\left(h \overline{2 \mathrm{~h}} \Theta_{\mathrm{j}} \equiv\left(h \overline{2 \mathrm{~h}} \Theta_{\mathrm{k}}\right.\right.$} \\
\hline $\mathrm{i}$ & $\eta_{4 \mathrm{~A}}$ & $\eta_{4 \mathrm{~B}}$ & $\eta_{4 \mathrm{C}}$ & $\eta_{4 \mathrm{D}}$ \\
\hline$j$ & $\eta_{4 \mathrm{G}}$ & $\eta_{4 \mathrm{E}}$ & $\eta_{4 \mathrm{~F}}$ & $\eta_{4 \mathrm{H}}$ \\
\hline $\mathrm{k}$ & $\eta_{4 \mathrm{~J}}$ & $\eta_{4 \mathrm{~K}}$ & $\eta_{4 \mathrm{I}}$ & $\eta_{4 \mathrm{~L}}$ \\
\hline
\end{tabular}


$\left(\eta_{5}, \eta_{5}\right)$

General:

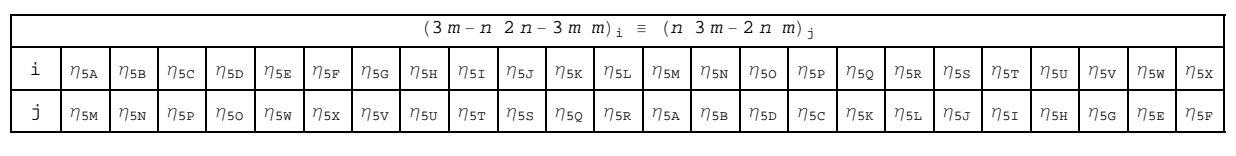

Special:

\begin{tabular}{|c|c|c|c|c|}
\hline \multicolumn{5}{|c|}{ 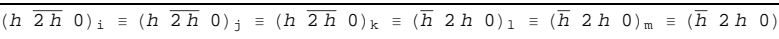 } \\
\hline$i$ & $\eta_{5 \mathrm{~A}}$ & $\eta_{5 B}$ & $\eta_{5 c}$ & $\eta_{50}$ \\
\hline$j$ & $\eta_{56}$ & $\eta_{5 E}$ & $\eta_{5 F}$ & $\eta_{5 \mathrm{H}}$ \\
\hline k & $\eta_{5 \mathrm{~J}}$ & $\eta_{5 \mathrm{~K}}$ & $\eta_{5 I}$ & $\eta_{5 L}$ \\
\hline 1 & $\eta_{5 \mathrm{M}}$ & $\eta_{5 \mathrm{~N}}$ & $\eta_{5 P}$ & $\eta_{50}$ \\
\hline $\mathrm{m}$ & $\eta_{5 \mathrm{~s}}$ & $\eta_{50}$ & $\eta_{5 T}$ & $\eta_{5 R}$ \\
\hline $\mathrm{n}$ & $\eta_{5 \mathrm{~V}}$ & $\eta_{5 \mathrm{~W}}$ & $\eta_{5 x}$ & $\eta_{50}$ \\
\hline
\end{tabular}

\begin{tabular}{|c|c|c|c|c|c|c|}
\hline \multicolumn{6}{|c|}{$(3 p \circ 2 p)_{\mathrm{i}} \equiv(3 p \circ 2 p)_{\mathrm{j}} \equiv(\overline{3 p} \odot \overline{2 p})_{\mathrm{k}} \equiv(\overline{3 p} \odot \overline{2 p})_{1}$} \\
\hline $\mathrm{i}$ & $\eta_{5 \mathrm{~A}}$ & $\eta_{5 \mathrm{C}}$ & $\eta_{5 \mathrm{E}}$ & $\eta_{5 \mathrm{G}}$ & $\eta_{5 \mathrm{I}}$ & $\eta_{5 \mathrm{~K}}$ \\
\hline $\mathrm{j}$ & $\eta_{5 \mathrm{M}}$ & $\eta_{5 \mathrm{P}}$ & $\eta_{5 \mathrm{~W}}$ & $\eta_{5 \mathrm{~V}}$ & $\eta_{5 \mathrm{~T}}$ & $\eta_{5 \mathrm{Q}}$ \\
\hline $\mathrm{k}$ & $\eta_{5 \mathrm{~B}}$ & $\eta_{5 \mathrm{D}}$ & $\eta_{5 \mathrm{~F}}$ & $\eta_{5 \mathrm{H}}$ & $\eta_{5 \mathrm{~J}}$ & $\eta_{5 \mathrm{~L}}$ \\
\hline $\mathrm{l}$ & $\eta_{5 \mathrm{~N}}$ & $\eta_{50}$ & $\eta_{5 \mathrm{X}}$ & $\eta_{5 \mathrm{U}}$ & $\eta_{5 \mathrm{~S}}$ & $\eta_{5 \mathrm{R}}$ \\
\hline
\end{tabular}

$\left(\eta_{6}, \eta_{6}\right)$

General:

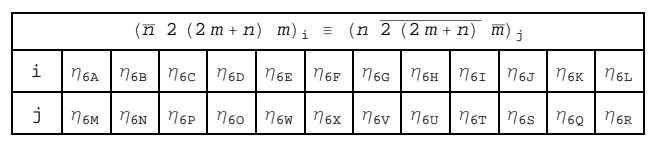

Special:

\begin{tabular}{|c|c|c|c|c|}
\hline \multicolumn{5}{|c|}{ 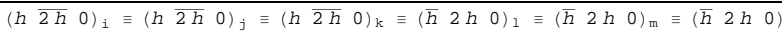 } \\
\hline$i$ & $\eta_{6 \mathrm{~A}}$ & $\eta_{6 \mathrm{~B}}$ & $\eta_{60}$ & $\eta_{60}$ \\
\hline${ }^{j}$ & $\eta_{6 G}$ & $\eta_{6 \mathrm{E}}$ & $\eta_{6 F}$ & $\eta_{6 \mathrm{H}}$ \\
\hline $\mathrm{k}$ & $\eta_{6 \mathrm{~J}}$ & $\eta_{6 \mathrm{~K}}$ & $\eta_{61}$ & $\eta_{6 L}$ \\
\hline 1 & $\eta_{6 M}$ & $\eta_{6 \mathrm{~N}}$ & $\eta_{6 P}$ & $\eta_{60}$ \\
\hline $\mathrm{m}$ & $\eta_{6 \mathrm{~S}}$ & $\eta_{60}$ & $\eta_{61}$ & $\eta_{6 \mathrm{R}}$ \\
\hline $\mathrm{n}$ & $\eta_{6 \mathrm{~V}}$ & $\eta_{6 \mathrm{~W}}$ & $\eta_{6 x}$ & $\eta_{60}$ \\
\hline
\end{tabular}

$\left(\eta_{7}, \eta_{7}\right)$

General:

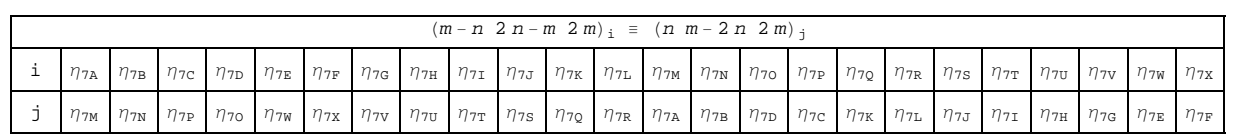

Special:

\begin{tabular}{|c|c|c|c|c|}
\hline \multicolumn{5}{|c|}{ 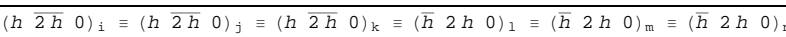 } \\
\hline$i$ & $\eta_{7 \mathrm{~A}}$ & $\eta_{78}$ & $\eta_{7 \mathrm{C}}$ & $\eta_{70}$ \\
\hline j & $\eta_{7 G}$ & $\eta_{7 \mathrm{E}}$ & $\eta_{7 \mathrm{~F}}$ & $\eta_{7 \mathrm{H}} \mathrm{-r}-\mathrm{rar}$ \\
\hline $\mathrm{k}$ & $\eta_{7 \mathrm{~J}}$ & $\eta_{7 \mathrm{~K}}$ & $\eta_{7 \mathrm{I}}$ & $\eta_{7 L}$ \\
\hline 1 & $\eta_{7 M}$ & $\eta_{7 \mathrm{~N}}$ & $\eta_{7 \mathrm{P}}$ & $\eta_{70}$ \\
\hline $\mathrm{m}$ & $\eta_{7 \mathrm{~s}}$ & $\eta_{70}$ & $\eta_{7 \mathrm{~T}}$ & $\eta_{7 R}$ \\
\hline $\mathrm{n}$ & $\eta_{7 \mathrm{~V}}$ & $\eta_{7 \mathrm{~W}}$ & $\eta_{7 x}$ & $\eta_{7 \mathrm{u}}$ \\
\hline
\end{tabular}

\begin{tabular}{|c|c|c|c|c|c|c|}
\hline \multicolumn{7}{|c|}{$(h \odot 4 h)_{\mathrm{i}} \equiv(h \odot 4 h)_{\mathrm{j}} \equiv(h \odot 4 h)_{\mathrm{k}} \equiv(h \odot 4 h)_{1}$} \\
\hline$i$ & $\eta_{7 \mathrm{~A}}$ & $\eta_{7 c}$ & $\eta_{7 E}$ & $\eta_{76}$ & $\eta_{7 I}$ & $\eta_{7 \mathrm{~K}}$ \\
\hline$j$ & $\eta_{7 M}$ & $\eta_{7 \mathrm{P}}$ & $n_{7 \mathrm{w}}$ & $n_{7 \mathrm{~V}}$ & $\eta_{7 T}$ & $\eta_{70}$ \\
\hline k & $\eta_{7 \mathrm{~B}}$ & $n_{70}$ & $n_{7 \mathrm{~F}}$ & $\eta_{7 \mathrm{H}}$ & $\eta_{7 \mathrm{~J}}$ & $\eta_{7 L}$ \\
\hline 1 & $\eta_{7 \mathrm{~N}}$ & $\eta_{70}$ & $\eta_{7 x}$ & $\eta_{70}$ & $\eta_{7 \mathrm{~s}}$ & $\eta_{7 \mathrm{R}}$ \\
\hline
\end{tabular}


$\left(\eta_{8}, \eta_{8}\right)$

Special:

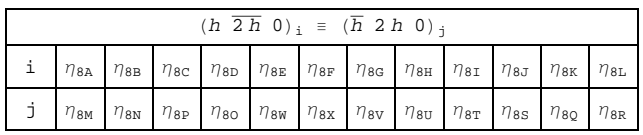

\begin{tabular}{|c|c|c|c|c|c|c|c|c|c|c|c|c|}
\hline \multicolumn{13}{|c|}{$\left(\begin{array}{llll}0 & \odot & I\end{array}\right)_{i} \equiv\left(\begin{array}{llll}0 & 0 & I\end{array}\right)_{j}$} \\
\hline i & $\eta_{8 \mathrm{~A}}$ & $\eta_{88}$ & $n_{8 c}$ & $\eta_{80}$ & $n_{8 E}$ & $\eta_{8 \mathrm{~F}}$ & $\eta_{86}$ & $\eta_{8 \mathrm{H}}$ & $\eta_{81}$ & $\eta_{8 \mathrm{~J}}$ & $\eta_{8 \mathrm{~K}}$ & $\eta_{8 L}$ \\
\hline & $\eta_{8 R}$ & $\eta_{8 \mathrm{~T}}$ & $\eta_{8 Q}$ & $\eta_{85}$ & $\eta_{80}$ & $\eta_{8 M}$ & $\eta_{8 \mathrm{P}}$ & $\eta_{8 \mathrm{~N}}$ & $\eta_{80}$ & $\eta_{8 \mathrm{w}}$ & $78 \mathrm{v}$ & $\eta_{8 x}$ \\
\hline
\end{tabular}

$\left(\eta_{9}, \eta_{9}\right)$

Special:

\begin{tabular}{|c|c|c|c|}
\hline \multicolumn{4}{|c|}{$\left(h 2 \overline{2} \theta_{i} \equiv(\hbar 2 h)_{j}\right.$} \\
\hline$i$ & $\eta_{9 A}$ & $\eta_{9 C}$ & $\eta_{9 E}$ \\
\hline$j$ & $\eta_{9 B}$ & $\eta_{9 D}$ & $\eta_{9 F}$ \\
\hline
\end{tabular}

$\left(\eta_{10}, \eta_{10}\right)$

General:

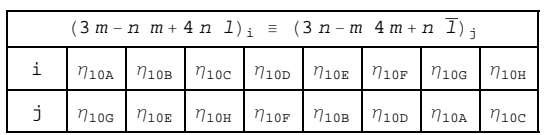

Special:

$(2 p 5 p \theta)_{\mathrm{i}} \equiv(2 p 5 p \theta)_{j} \equiv(\overline{2 p} 5 p \quad 0)_{\mathrm{K}} \equiv(\overline{2 p} 5 p \quad 0)_{1}$

\begin{tabular}{|c|c|c|}
\hline$i$ & $\eta_{1 \mathrm{AA}}$ & $\eta_{1 \mathrm{OB}}$ \\
\hline$j$ & $\eta_{1 \mathrm{OG}}$ & $\eta_{1 \mathrm{OE}}$ \\
\hline $\mathrm{k}$ & $\eta_{1 \mathrm{OD}}$ & $\eta_{1 \mathrm{OC}}$ \\
\hline 1 & $\eta_{1 \mathrm{OF}}$ & $\eta_{1 \mathrm{OH}}$ \\
\hline
\end{tabular}

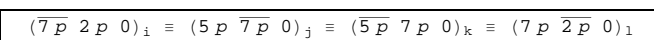

\begin{tabular}{|c|c|c|c|c|}
\hline$i$ & $\eta_{1 \mathrm{AA}}$ & $\eta_{1 \mathrm{OB}}$ & $\eta_{1 \mathrm{OE}}$ & $\eta_{1 \mathrm{OF}}$ \\
\hline$j$ & $\eta_{1 \mathrm{OG}}$ & $\eta_{1 \mathrm{OE}}$ & $\eta_{1 \mathrm{OB}}$ & $\eta_{1 \mathrm{OD}}$ \\
\hline $\mathrm{k}$ & $\eta_{1 \mathrm{OH}}$ & $\eta_{1 \mathrm{OF}}$ & $\eta_{1 \mathrm{OA}}$ & $\eta_{1 \mathrm{OC}}$ \\
\hline 1 & $\eta_{1 \mathrm{OC}}$ & $\eta_{1 \mathrm{OD}}$ & $\eta_{1 \mathrm{OG}}$ & $\eta_{1 \mathrm{OH}}$ \\
\hline
\end{tabular}

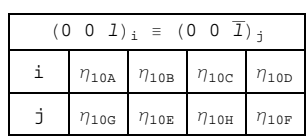


$\left(\eta_{11}, \eta_{11}\right)$

Special:

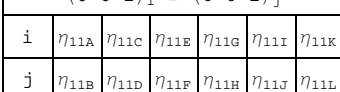

\begin{tabular}{|c|c|c|c|c|}
\hline \multicolumn{4}{|c|}{$(h \odot)_{\mathrm{i}} \equiv(h \odot \Theta)_{j} \equiv(h \odot \Theta)_{\mathrm{k}}$} \\
\hline $\mathrm{i}$ & $\eta_{11 \mathrm{~A}}$ & $\eta_{11 \mathrm{~B}}$ & $\eta_{11 \mathrm{C}}$ & $\eta_{11 \mathrm{D}}$ \\
\hline $\mathrm{j}$ & $\eta_{11 \mathrm{G}}$ & $\eta_{11 \mathrm{E}}$ & $\eta_{11 \mathrm{~F}}$ & $\eta_{11 \mathrm{H}}$ \\
\hline $\mathrm{k}$ & $\eta_{11 \mathrm{I}}$ & $\eta_{11 \mathrm{~K}}$ & $\eta_{11 \mathrm{I}}$ & $\eta_{11 \mathrm{~L}}$ \\
\hline
\end{tabular}

$\left(\eta_{12}, \eta_{12}\right)$

Special:

\begin{tabular}{|c|c|c|c|}
\hline \multicolumn{4}{|c|}{ 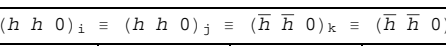 } \\
\hline$i$ & $\eta_{12 \mathrm{~A}}$ & $\eta_{12 c}$ & $\eta_{12 E}$ \\
\hline$j$ & $\eta_{12 \mathrm{H}}$ & $\eta_{12 \mathrm{~J}}$ & $\eta_{12 L}$ \\
\hline k & $\eta_{12 \mathrm{~B}}$ & $\eta_{12 D}$ & $\eta_{12 \mathrm{~F}}$ \\
\hline 1 & $\eta_{12 G}$ & $\eta_{12 \mathrm{I}}$ & $\eta_{12 \mathrm{~K}}$ \\
\hline
\end{tabular}


(ii) : $\left(\eta_{i}, \eta_{j}\right)$

$\left(\eta_{1}, \eta_{3}\right)$

\begin{tabular}{|c|c|c|c|}
\hline$\eta_{1 \mathrm{~A}}$ & \begin{tabular}{l|l}
$\eta_{3 A}$ & $\eta_{3 B} B$ \\
\end{tabular} & $n_{3 c}$ & $\eta_{30}$ \\
\hline$h \overline{2 h} \theta)$ & \begin{tabular}{l|l|} 
& $(\bar{h} \bar{h} \Theta)$ \\
\end{tabular} & & $\overline{h \overline{2 h} \theta)}$ \\
\hline$\eta_{1 B}$ & $\eta_{3 A}$ & $\eta_{3 c}$ & $\eta_{30}$ \\
\hline$(h \overline{2 h} \theta)$ & $\left(\begin{array}{lll}h & h & \theta\end{array}\right)$ & $\left(\begin{array}{ll}h & 2 h\end{array}\right.$ & h o) \\
\hline$\eta_{1 c}$ & $\eta_{3 A}$ & $\eta_{3 B}$ & \begin{tabular}{l|l|l|l|l}
$\eta_{3 c}$ & $\eta_{30}$ \\
\end{tabular} \\
\hline$(h \overline{2 h} \theta)$ & \begin{tabular}{|l|l|}
$(2 h h \theta)$ & $(21$ \\
\end{tabular} & $\overline{h \bar{h} \theta)}$ & \\
\hline$\eta_{10}$ & \begin{tabular}{|l|l}
$\eta_{3 A}$ & $\eta_{3 B}$
\end{tabular} & & $\eta_{30}$ \\
\hline$(h \overline{2 h} \theta)$ & $\overline{2 h}$ & $h 0)$ & $(2 h \bar{h} \theta)$ \\
\hline$\eta_{1 E}$ & $\eta_{3 B}$ & \multirow{2}{*}{\multicolumn{2}{|c|}{\begin{tabular}{|c|l|}
$\eta_{3} c$ & $\eta_{30}$ \\
\end{tabular}}} \\
\hline$(h \overline{2 h} \theta)$ & $\left(\begin{array}{lll}h & 2 h & h\end{array}\right)$ & \begin{tabular}{|l|l|l|}
$(h \quad h \quad$ & \\
\end{tabular} & \\
\hline$\eta_{1 F}$ & \begin{tabular}{l|l}
$\eta_{3 A}$ & $\eta_{3 B}$ \\
\end{tabular} & \begin{tabular}{|l|l|}
$B$ & $\eta_{3} c$ \\
\end{tabular} & $\eta_{30}$ \\
\hline$(h \overline{2 h} \theta)$ & $(\bar{h} 2 h \quad \theta)$ & & $(h \quad h \quad \theta)$ \\
\hline
\end{tabular}

$\left(\eta_{1}, \eta_{4}\right)$

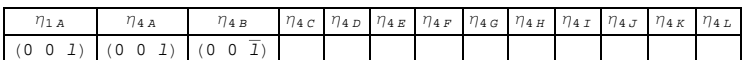
\begin{tabular}{|l|l|l|l|l|l|l|l|l|l|l|l|l|}
\hline$\eta_{18}$ & $\eta_{4 A}$ & $\eta_{48}$ & $\eta_{4 C}$ & $\eta_{40}$ & $\eta_{46}$ & $\eta_{4 F}$ & $\eta_{46}$ & $\eta_{44}$ & $\eta_{44}$ & $\eta_{44}$ & $\eta_{44}$ & $\eta_{4 L}$ \\
\hline
\end{tabular}

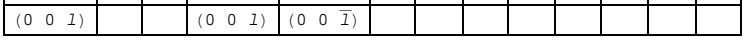

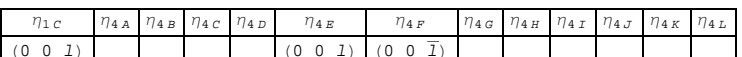

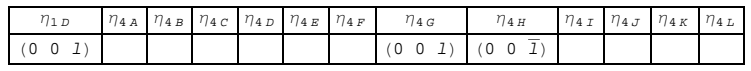
\begin{tabular}{|c|c|c|c|c|c|c|c|c|c|c|c|c|}
\hline$\eta_{1 E}$ & $\eta_{4 A}$ & $\eta_{4 B}$ & $\eta_{4 C}$ & $\eta_{40}$ & $\eta_{4 E}$ & $\eta_{4 F}$ & $\eta_{46}$ & $\eta_{4 H}$ & $\eta_{4 I}$ & $\eta_{4 J}$ & $\eta_{4 K}$ & $\eta_{4 L}$ \\
\hline$(\Theta \Theta \Theta 1)$ & & & & & & & & & $(\Theta \Theta O)$ & $(\Theta \Theta O \bar{I})$ & & \\
\hline
\end{tabular}

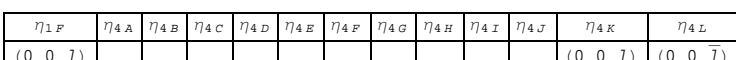

$\left(\eta_{1}, \eta_{9}\right)$

\begin{tabular}{|c|c|c|c|c|c|c|c|}
\hline$\frac{\eta_{1 \mathrm{~A}}}{(\theta 001)}$ & & & $\eta_{98}$ & $\eta_{9 c}$ & $n_{90}$ & 796 & $\eta_{9 F}$ \\
\hline$\frac{\eta_{18}}{(\theta 001)}$ & $\eta_{9 \mathrm{~A}}$ & & $\frac{9 B}{91)}$ & $\eta_{9 c}$ & $\eta_{90}$ & $7_{91}$ & $\eta_{9 F}$ \\
\hline$\frac{\eta_{1 c}}{(\theta \theta 01)}$ & $\eta_{9 \mathrm{~A}}$ & \begin{tabular}{|l|}
$\eta_{9 \mathrm{~B}}$ \\
\end{tabular} & $\frac{\eta}{10}$ & $\frac{c}{61)}$ & 790 & $7_{96}$ & $\eta_{9 F}$ \\
\hline$\frac{\eta_{10}}{(\theta \Theta \theta 1)}$ & $\eta_{9 \mathrm{~A}}$ & $\eta_{9 \mathrm{BB}}$ & $\eta_{9 \mathrm{c}}$ & & $\begin{array}{l}\frac{90}{61)} \\
61)\end{array}$ & $\eta_{91}$ & $\eta_{9 F}$ \\
\hline \begin{tabular}{|c|c|}
$\eta_{1 E}$ \\
$\left(\begin{array}{lll}\theta & 1\end{array}\right)$
\end{tabular} & $\eta_{9 \mathrm{~A}}$ & $\eta_{9 \mathrm{~B}}$ & $\eta_{9 \mathrm{c}}$ & $\eta_{90}$ & & $\frac{E}{61)}$ & $\eta_{9 F}$ \\
\hline \begin{tabular}{|c|c}
$\eta_{1 F}$ \\
$(\theta \ominus 1)$
\end{tabular} & $\eta_{9 \mathrm{~A}}$ & $\eta_{9 B}$ & $\eta_{9 c}$ & $\eta_{90}$ & $\eta_{9 \mathrm{E}}$ & & $\begin{array}{l}9 F \\
\theta 1)\end{array}$ \\
\hline
\end{tabular}

$\left(\eta_{1}, \eta_{11}\right)$

\begin{tabular}{|c|c|c|c|c|c|c|c|c|c|c|c|c|c|c|}
\hline$\eta_{1 A}$ & $\eta_{11}$ & & $\begin{array}{l}\eta_{118} \\
\end{array}$ & 711 & \begin{tabular}{ll|l}
10 & $\eta_{11}$ \\
\end{tabular} & \begin{tabular}{l|l}
10 & $\eta_{11}$ \\
\end{tabular} & \begin{tabular}{l|l}
$11 E$ & $\eta_{11}$ \\
\end{tabular} & $\mid 11 F$ & \begin{tabular}{l|l}
116 & 71 \\
\end{tabular} & 1114 & 711I & $711 \mathrm{~J}$ & $\eta_{11 k}$ & $\eta_{11 L}$ \\
\hline $\begin{array}{llll}0 & 011\end{array}$ & $\left(\begin{array}{lll}0 & 0\end{array}\right.$ & & 001 & & & & & & & & & & & \\
\hline$\eta_{1 B}$ & $\eta_{11 A}$ & $\eta_{11 B}$ & $\eta_{11}$ & & $\eta_{110}$ & $\eta_{11}$ & \begin{tabular}{l|l}
$11 E$ & $\eta_{11}$
\end{tabular} & \begin{tabular}{l|l}
$11 F$ & $\eta_{11}$
\end{tabular} & \begin{tabular}{l|l}
116 & $\eta \exists$
\end{tabular} & $111 \mathrm{H}$ & $711 \mathrm{I}$ & $\eta_{111}$ & $\eta_{11 k}$ & $\eta_{11 L}$ \\
\hline 001 (1) & & & $(\theta)$ & 1) & 1001 & & & & & & & & & \\
\hline$\eta_{1 c}$ & $\eta_{11 \mathrm{~A}}$ & $\eta_{118}$ & $\eta_{11 c}$ & $\eta_{110}$ & $\eta_{11}$ & & $\eta_{11 F}$ & & $l_{116} \mid$ & 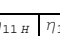 & $\overline{\eta_{111}}$ & $\bar{n}_{11,}$ & $\eta_{11 K}$ & $\eta_{11 L}$ \\
\hline $\begin{array}{llll}0 & 0 & 1\end{array}$ & & & & & $\left(\begin{array}{lll}0 & 0\end{array}\right.$ & 1) & $(001$ & $\bar{I})$ & & & & & & \\
\hline$\eta_{10}$ & $\eta_{11 A}$ & $\eta_{118}$ & $\eta_{11 c}$ & $\eta_{110}$ & $n_{11 E}$ & $\eta_{11 F}$ & $\eta_{11}$ & & $\eta_{11 !}$ & & $\overline{\eta_{111}}$ & $\mid \eta_{11,}$ & $\mid \eta_{11 k}$ & $\eta_{1114}$ \\
\hline$\left(\begin{array}{lll}(0 & 0 & 1\end{array}\right)$ & & & & & & & $\begin{array}{ll}10 \theta \\
\theta 0\end{array}$ & \begin{tabular}{l|l}
$01)$ & $(1$
\end{tabular} & 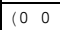 & $\bar{I})$ & & & & \\
\hline$\eta_{1 E}$ & $\eta_{11 A}$ & $\eta_{11 B}$ & $\eta_{11 c}$ & $\eta_{110}$ & $\eta_{11 E}$ & $\eta_{11 F}$ & $\eta_{11 G}$ & $\eta_{11 \mathrm{H}}$ & & $11 I$ & & $11 \mathrm{~J}$ & $\eta_{11 k}$ & $\eta_{11 L}$ \\
\hline $\begin{array}{llll}0 & 0 & 1)\end{array}$ & & & & & & & & & & $\begin{array}{lll}\theta & 1) \\
\end{array}$ & 10 & $\theta \bar{I})$ & & \\
\hline$\eta_{1 F}$ & $\eta_{11 A}$ & $\eta_{11 \mathrm{~B}}$ & $\eta_{11 c}$ & $\eta_{110}$ & $\eta_{11 E}$ & $\eta_{11 F}$ & $\eta_{11 \mathrm{G}}$ & $\eta_{11 \mathrm{H}}$ & \begin{tabular}{l|l}
$H$ & $\eta_{111}$
\end{tabular} & \begin{tabular}{l|l}
$I$ & $\eta_{111}$ \\
\end{tabular} & 10 & $\eta_{11 k}$ & & $711 \mathrm{~L}$ \\
\hline$\left(\begin{array}{lll}0 & 0 & 1\end{array}\right)$ & & & & & & & & & & & & $\begin{array}{lll}0 & 11\end{array}$ & (e) & $\theta \bar{I})$ \\
\hline
\end{tabular}


$\left(\eta_{2}, \eta_{3}\right)$

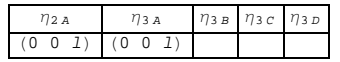

\begin{tabular}{|c|c|c|c|c|}
\hline$\eta_{2 B} B$ & $\eta_{3 A}$ & $\eta_{3 B} B$ & $\eta_{3 C}$ & $\eta_{30}$ \\
\hline$(0 \Theta 1)$ & & $(001)$ & & \\
\hline
\end{tabular}

\begin{tabular}{|c|c|c|c|c|}
\hline$\eta_{2 C} C$ & $\eta_{3 A}$ & $\eta_{3 B}$ & $\eta_{3 C}$ & $\eta_{30}$ \\
\hline$(0001)$ & & & $(001)$ & \\
\hline
\end{tabular}

\begin{tabular}{|c|c|c|c|c|}
\hline$\eta_{20}$ & $\eta_{3 A}$ & $\eta_{3 B}$ & $\eta_{3 C}$ & $\eta_{30}$ \\
\hline$(\theta \theta 1)$ & & & & $(\theta \theta 1)$ \\
\hline
\end{tabular}

$\left(\eta_{2}, \eta_{8}\right)$

\begin{tabular}{|c|c|c|c|c|c|c|c|c|c|c|c|c|c|c|c|c|c|c|c|c|c|c|c|c|c|c|c|c|c|c|}
\hline$\eta_{2 A}$ & \multicolumn{2}{|c|}{$\eta_{8 \mathrm{~A}}$} & 788 & \begin{tabular}{|l|l|}
$78 c$ \\
\end{tabular} & 780 & $\eta_{8 E}$ & $7_{8 F}$ & \multicolumn{2}{|c|}{$\eta_{86}$} & \begin{tabular}{|l|}
$78 \mathrm{H}$ \\
\end{tabular} & \begin{tabular}{|l|}
$78 I$ \\
\end{tabular} & \multirow{2}{*}{\multicolumn{2}{|c|}{$78 \mathrm{~J}$}} & & \multirow{2}{*}{\multicolumn{2}{|c|}{$78 \mathrm{M}$}} & $\eta_{8 \mathrm{~N}}$ & 780 & $78 P$ & $\eta_{80}$ & $n_{8 R}$ & \multirow{2}{*}{\multicolumn{2}{|c|}{$\eta_{8 \mathrm{~s}}$}} & $\eta_{8 T}$ & & \multirow{2}{*}{\multicolumn{2}{|c|}{\begin{tabular}{|c|}
$\eta_{8 \mathrm{~V}}$ \\
$(\bar{h} 2 \mathrm{~h} \theta$
\end{tabular}}} & \multicolumn{2}{|c|}{\begin{tabular}{|l|l|}
$\eta_{8}$ & $\eta_{8 x} x$ \\
\end{tabular}} \\
\hline$\left(\begin{array}{lll}h & h & \theta\end{array}\right)$ & & & & & & & & $(h \overline{2 t}$ & $\bar{h}$ ब) & & & & & & & & & & & & & & & & & & & & & \\
\hline$(h \overline{2 h} \theta)$ & $(h \overline{2}$ & 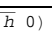 & & & & & & & & & & & & & & & $2 h \theta)$ & & & & & & & & & & & & & \\
\hline$(2 h \bar{h} \theta)$ & & & & & & & & & & & & (h) 2 & h $h$ ब) & & & & & & & & & & $\langle h \overline{2 h}$ & $\begin{array}{l}h \theta) \\
\end{array}$ & & & & & & \\
\hline$\eta_{2 B}$ & $\overline{B P A} \mid$ & $\eta_{8 B}$ & $\overline{78 c}$ & $\eta_{80}$ & & $\overline{\bar{B}_{8 E}}$ & $\eta_{8 F}$ & $\eta_{86}$ & $\eta_{8 \mathrm{H}}$ & & $\bar{\eta}_{8 I}$ & $n_{8 \mathrm{~J}}$ & $7_{8 K}$ & $\overline{78 L}_{81}$ & & $\overline{\eta_{8 M}}$ & $\overline{\eta_{8 N}}$ & $\bar{\eta}_{8}$ & & $\overline{\eta_{8 P} p}$ & 780 & & $78 R$ & $\eta_{8 s}$ & $\eta_{8 T}$ & & $\overline{780}$ & $\eta_{8 v}$ & $n_{8 w}$ & $778 x$ \\
\hline$\left(\begin{array}{ll}(h \quad h \quad & \theta\end{array}\right)$ & & & & & & & & & $\langle h \overline{2 h}$ & & & & & & & & & & & & & & & & & & $2 h \theta$ & & & \\
\hline$(h \overline{2 h} \theta)$ & & & & $h \overline{2 h}$ & & & & & & & & & & & & & & (h) 2 & $h$ ब) & & & & & & & & & & & \\
\hline$(2 h \bar{h} \theta)$ & & & & & & & & & & & & & & $\overline{k n} 2 h$ & $h \theta$ & & & & & & & & $2 \overline{2 h} \theta$ & & & & & & & \\
\hline$\eta_{2 c}$ & $\overline{7}_{8 A}$ & $\eta_{\mathrm{g}}$ & $B$ & $\eta_{8 \mathrm{c}}$ & 780 & & $8 E$ & $n_{8 F}$ & $\eta_{86}$ & \begin{tabular}{|l|}
$78 \mathrm{H}$ \\
\end{tabular} & \begin{tabular}{|l|}
$78 I$ \\
\end{tabular} & $\eta_{80}$ & 781 & $\beta_{k}$ & $\eta_{8 L}$ & $\eta_{8 M}$ & & $8 \mathrm{~N}$ & 780 & $78 \mathrm{P}$ & & 789 & \begin{tabular}{|l|l|}
$7_{8 R}$ \\
\end{tabular} & $78 s$ & $78 T$ & \begin{tabular}{|l|}
780 \\
\end{tabular} & $n_{8 V}$ & $\eta_{81}$ & $8 W$ & $\eta_{8 x}$ \\
\hline $\begin{array}{l}(h \quad h \quad \theta) \\
\end{array}$ & & & & & & $\langle\bar{h} 2$ & (h) & & & & & & & & & & & & & & & & & & & & & $(h \overline{2 h}$ & (h ब) & \\
\hline$(h \overline{2 h} \theta)$ & & & & & & & & & & & & & $\langle\bar{h} 2 h$ & $h \Theta$ & & & & & & & $\langle h \overline{2}$ & $2 h \quad 0)$ & & & & & & & & \\
\hline$(2 h \bar{h} \theta)$ & & $(h \overline{2}$ & (h⿻) & & & & & & & & & & & & & & $(\bar{h} 2$ & $2 h 0)$ & & & & & & & & & & & & \\
\hline$\eta_{2 D}$ & $\eta_{8 A}$ & $\bar{n}_{88}$ & $\eta_{88}$ & & $\eta_{80}$ & $78 \mathrm{E}$ & $\eta_{8}$ & & 786 & $\eta_{8 \mathrm{H}}$ & & $78 \mathrm{I}$ & \begin{tabular}{|l|l|}
$78 \mathrm{~J}$ & \\
\end{tabular} & $78 \mathrm{k}$ & $78 L$ & $78 \mathrm{M}$ & $7_{8 N}$ & 780 & $78 \mathrm{P}$ & $8 p$ & 780 & $78 R$ & $78 s$ & $\eta_{8 T}$ & & 780 & $78 \mathrm{~V}$ & $78 \mathrm{w}$ & $\eta_{8 \lambda}$ & \\
\hline$(h h \theta)$ & & & & & & & (h 2 & $h \theta$ & & & & & & & & & & & & & & & & & & & & & & $\bar{h} 0)$ \\
\hline$(h \overline{2 h} \theta)$ & & & & & & & & & & & & $2 h \quad 0)$ & & & & & & & & & & & & $(h \overline{2 h}$ & (h) & & & & & \\
\hline$(2 h \bar{h} \Theta)$ & & & $(h \overline{2 h}$ & & & & & & & & & & & & & & & & $(\bar{h} 2 h$ & 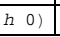 & & & & & & & & & & \\
\hline
\end{tabular}

$\left(\eta_{2}, \eta_{9}\right)$

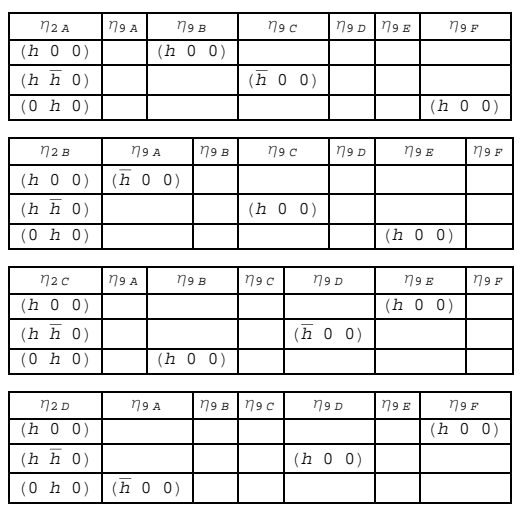

$\left(\eta_{2}, \eta_{10}\right)$

\begin{tabular}{|c|c|c|c|c|c|c|c|c|c|c|}
\hline \multirow{2}{*}{$\begin{array}{c}\eta_{2 A} \\
(0 \quad \theta \quad 1)\end{array}$} & \multirow{2}{*}{\multicolumn{2}{|c|}{$\begin{array}{l}\eta_{10 A} \\
\left(\begin{array}{llll}\theta & 0 & 1\end{array}\right)\end{array}$}} & $\eta_{108}$ & $\eta_{10 c}$ & $\eta_{100}$ & $\eta_{10 E}$ & $\eta_{10 F}$ & $\eta_{1}$ & & $\eta_{10 \mathrm{H}}$ \\
\hline & & & & & & & & \multicolumn{2}{|c|}{$\left(\begin{array}{ll}\theta & \theta\end{array}\right)$} & \\
\hline$\eta_{2 B}$ & $\overline{\eta_{10} \mathrm{~A}}$ & $\bar{\eta}_{10 B}$ & $\eta_{10}$ & & 100 & $\eta_{10 E}$ & $\eta_{10}$ & & $\overline{\eta_{100}}$ & $\eta_{10 \mathrm{H}}$ \\
\hline$\left(\begin{array}{llll}(0) & 1\end{array}\right)$ & & & & & 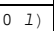 & & & I) & & \\
\hline$\eta_{2 c}$ & $\eta_{10 \mathrm{~A}}$ & $\eta_{10}$ & & $\eta_{110 c}$ & $\eta_{10}$ & $\eta_{11}$ & & $10 \mathrm{~F}$ & $\eta_{1106}$ & $\eta_{10 \mathrm{H}}$ \\
\hline$\left(\begin{array}{llll}0 & 0 & 1)\end{array}\right.$ & & $\begin{array}{lll}0 \theta & 0 \\
\end{array}$ & & & & $\begin{array}{lll}\theta \theta \\
\end{array}$ & 1) & & & \\
\hline$\eta_{20}$ & $\eta_{10 \mathrm{~A}}$ & $\eta_{10 B}$ & & $10 \mathrm{c}$ & $\eta_{10}$ & $\overline{\eta 10 E}$ & $\eta_{10 \mathrm{~F}}$ & $\eta_{100}$ & & $110 \mathrm{H}$ \\
\hline$\left(\begin{array}{lll}0 & 0 & 1\end{array}\right)$ & & & & $\begin{array}{ll}\theta \bar{I}) \\
\end{array}$ & & & & & & 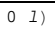 \\
\hline
\end{tabular}

$\left(\eta_{2}, \eta_{11}\right)$

\begin{tabular}{|c|c|c|c|c|c|c|c|c|c|c|c|c|c|c|c|}
\hline$\eta_{2 A}^{A}$ & \multicolumn{2}{|c|}{$\eta_{11 \mathrm{~A}}$} & $\eta_{11 B}$ & $\eta_{11 c}$ & $\eta_{110}$ & $\eta_{11 E}$ & $\eta_{11 F}$ & \multirow{2}{*}{\multicolumn{2}{|c|}{$\eta_{116}$}} & \begin{tabular}{|l|}
$\eta_{11 H}$ \\
\end{tabular} & $\eta_{11 I}$ & \multirow{2}{*}{\multicolumn{2}{|c|}{$\eta_{11 \mathrm{~J}}$}} & \multicolumn{2}{|c|}{\begin{tabular}{l|l}
$11 \mathrm{~K}$ & $\eta_{111}$ \\
\end{tabular}} \\
\hline$\langle(h h \theta)$ & & & & & & & & (h) 2 & & & & & & & \\
\hline$(h \overline{2 h} \theta)$ & $\sqrt{\bar{n} 2 h}$ & & & & & & & & & & & & & & \\
\hline$(2 h \hbar \theta)$ & & & & & & & & & & & & $\langle h \overline{2}$ & 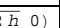 & & \\
\hline$\eta_{2 B}$ & $\eta_{11 A}$ & $\eta_{11 B}$ & $\eta_{11 c}$ & $\eta_{11}$ & & $\eta_{11 E}$ & $\eta_{11 F}$ & $\eta_{116}$ & $\eta_{1}$ & $11 \mathrm{H}$ & $\eta_{11 I}$ & $\eta_{11 \mathrm{~J}}$ & $\eta_{11 k}$ & & $\overline{114}$ \\
\hline 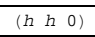 & & & & & & & & & (5) 2 & 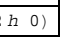 & & & & & \\
\hline$(h \overline{2 h} \theta)$ & & & & (h) 2 & $h \theta)$ & & & & & & & & & & \\
\hline$(2 h \hbar \theta)$ & & & & & & & & & & & & & & $\langle h \overline{2}$ & 250 \\
\hline$\eta_{2 c}$ & $\mid$ & $\bar{n}_{1}$ & & $\mid \eta_{11 c}$ & $\mid \begin{array}{l}n_{111} \\
\end{array}$ & $\overline{\eta_{1}}$ & & 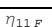 & $\mid \eta_{116}$ & $\eta_{11 \mathrm{H}}$ & $\eta_{111}$ & $\eta_{11,}$ & $\eta_{1}$ & & 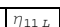 \\
\hline$(h h \theta)$ & & & & & & (h) $\overline{2}$ & & & & & & & & & \\
\hline$(h \overline{2 h} \theta)$ & & & & & & & & & & & & & $\langle h \overline{2}$ & $2 h$ ब) & \\
\hline$(2 h \hbar \theta)$ & & (h 2 & 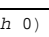 & & & & & & & & & & & & \\
\hline$\eta_{20}$ & $\eta_{11 \mathrm{~A}}$ & $\eta_{11 B}$ & & & $\eta_{110}$ & $\eta_{11 E}$ & $\eta_{1}$ & & $\eta_{116}$ & $\eta_{11 H}$ & $\eta_{1}$ & 111 & 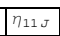 & $\eta_{11 k}$ & $\eta_{11 L}$ \\
\hline$\langle h \quad h \quad \theta)$ & & & & & & & & & & & & & & & \\
\hline$\left(\begin{array}{ll}(h 2 h & 0\end{array}\right)$ & & & & & & & & & & & $(h \overline{2}$ & $2 h$ o) & & & \\
\hline$(2 h \bar{\hbar} \theta)$ & & & $\sqrt{6} 2$ & $\begin{array}{ll}h \theta) \\
\end{array}$ & & & & & & & & & & & \\
\hline
\end{tabular}


$\left(\eta_{3}, \eta_{\mathrm{I}}\right)$

\begin{tabular}{|c|c|c|c|c|c|c|c|c|c|c|}
\hline$\eta_{3 A}$ & $\eta_{1 \mathrm{~A}}$ & \multicolumn{2}{|c|}{$\eta_{1 B}$} & \multicolumn{2}{|c|}{$\eta_{1 c}$} & $\eta_{10}$ & $\eta_{1 E}$ & \multicolumn{3}{|c|}{$\eta_{1 F}$} \\
\hline$(h \quad h \quad \Theta)$ & & \multicolumn{2}{|c|}{$(h \overline{2 h} \Theta)$} & & & & & \\
\hline$(h \overline{2 h} \theta)$ & & & & & & & & \multicolumn{2}{|c|}{$(\bar{h} 2 h 0$} & \\
\hline$(2 h \bar{h} \theta)$ & & & & \multicolumn{2}{|c|}{$(\bar{h} 2 h \theta)$} & & & & \\
\hline$\eta_{3 B}$ & \multicolumn{2}{|c|}{$\eta_{1 \mathrm{~A}}$} & $\eta_{1 B}$ & \multicolumn{2}{|c|}{$\eta_{1 c}$} & $\eta_{10}$ & \multicolumn{3}{|c|}{$\eta_{1 E}$} & $\eta_{1 F}$ \\
\hline$(h \quad h \quad \Theta)$ & \multicolumn{2}{|c|}{$(\bar{h} 2 h \theta)$} & & & & & & & & \\
\hline$\langle(h \overline{2 h} \theta)$ & & & & & & & \multicolumn{3}{|c|}{$\left(\begin{array}{llll}h & 2 h & \theta\end{array}\right)$} & \\
\hline$\left(\begin{array}{llll}2 h & \bar{h} & \Theta\end{array}\right)$ & & & & \multicolumn{2}{|c|}{$(h \overline{2 h} \theta)$} & & & & & \\
\hline$\eta_{3 c}$ & $\eta_{1 \mathrm{~A}}$ & \multicolumn{2}{|c|}{$\eta_{1 B}$} & $\eta_{1 c}$ & $\eta_{1}$ & & \multicolumn{3}{|c|}{$\eta_{1 E}$} & $\eta_{1 F}$ \\
\hline$\left\langle\begin{array}{lll}h & h & \theta\end{array}\right)$ & & & & & & & \multicolumn{3}{|c|}{$\langle h \overline{2 h} \theta\rangle$} & \\
\hline$\langle h \overline{2 h} \Theta\rangle$ & \multirow{2}{*}{\multicolumn{3}{|c|}{ 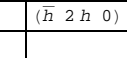 }} & & & & & & & \\
\hline$\left(\begin{array}{llll}2 h & \bar{h} & \Theta\end{array}\right)$ & & & & & \multicolumn{2}{|c|}{$\left(\begin{array}{lll}h & 2 h & \theta\end{array}\right)$} & & & & \\
\hline$\eta_{3 D}$ & \multicolumn{2}{|c|}{$\eta_{1 \mathrm{~A}}$} & $\eta_{1 B}$ & $\eta_{1 c}$ & \multicolumn{2}{|c|}{$\eta_{10}$} & $\eta_{1 E}$ & \multicolumn{3}{|c|}{$\eta_{1 F}$} \\
\hline$\left(\begin{array}{lll}h & h & \Theta\end{array}\right)$ & & & & & & & & \multicolumn{3}{|c|}{$(h \overline{2 h} \odot\rangle$} \\
\hline$(h \overline{2 h} \theta)$ & \multicolumn{3}{|c|}{$(h \overline{2 h} \Theta)$} & & & & & & & \\
\hline$\left(\begin{array}{lll}2 h & \hbar & 0\end{array}\right)$ & & & & & $(h \overline{2}$ & h0) & & & & \\
\hline
\end{tabular}

$\left(\eta_{3}, \eta_{2}\right)$

\begin{tabular}{|c|c|c|c|c|c|}
\hline$\frac{\eta_{3 A}}{(\theta \theta 01)}$ & \begin{tabular}{|r|}
$\eta_{2}$ \\
0
\end{tabular} & & $\eta_{28}$ & $\eta_{2 c}$ & $\eta_{20}$ \\
\hline$\frac{\eta_{38}}{\left(\begin{array}{llll}0 & 0 & 1\end{array}\right)}$ & $\eta_{2 A}$ & & 1) & $\eta_{2 c}$ & $\eta_{20}$ \\
\hline $\begin{array}{c}\eta_{3 c} \\
\left(\begin{array}{llll}0 & 0 & 1\end{array}\right)\end{array}$ & $\eta_{2 A}$ & $\eta_{2 B}$ & & $\frac{c}{1}$ & 720 \\
\hline$\frac{\eta_{30}}{(0 \quad 01)}$ & $\eta_{2 A}$ & $\eta_{2 B}$ & $\eta_{2 c}$ & & $\begin{array}{l}\frac{20}{011} \\
01)\end{array}$ \\
\hline
\end{tabular}

$\left(\eta_{3}, \eta_{4}\right)$

\begin{tabular}{|c|c|c|c|c|c|c|c|c|c|c|c|c|}
\hline$\eta_{3 \mathrm{~A}}$ & $\eta_{4 A}$ & $\eta_{48}$ & $7_{40}$ & $n_{40}$ & $7_{4 E}$ & $\eta_{4 F}$ & $\eta_{46}$ & $\eta_{4 H}$ & $n_{41}$ & $\eta_{4 J}$ & $\eta_{4 k}$ & $\eta_{4 L}$ \\
\hline$(h \circ \theta 0)$ & & & & & & & & & & $(h \odot \theta \Theta)$ & & \\
\hline$(h \bar{h} \Theta)$ & $(\bar{h} \odot \theta)$ & & & & & & & & & & & \\
\hline$\left(\begin{array}{lll}(\theta & h & \theta\end{array}\right)$ & & & & & & & $\bar{h} \odot \theta$ & & & & & \\
\hline
\end{tabular}

\begin{tabular}{|c|c|c|c|c|c|c|c|c|c|c|c|c|}
\hline$\eta_{3 B}$ & $\eta_{4 A}$ & $\eta_{4 B}$ & $\eta_{4 C}$ & $\eta_{4 D}$ & $\eta_{4 E}$ & $\eta_{4 F}$ & $\eta_{4 G}$ & $\eta_{4 H}$ & $\eta_{4 I}$ & $\eta_{4 J}$ & $\eta_{4 K}$ & $\eta_{4 L}$ \\
\hline$(h \odot \odot)$ & & & & & & & & & & & & $(h \odot \Theta)$ \\
\hline$(h \bar{h} \odot)$ & & & & $(\bar{h} \odot \odot)$ & & & & & & & & \\
\hline$(\odot h \odot)$ & & & & & & & & $(\bar{h} \odot \odot)$ & & & & \\
\hline
\end{tabular}

\begin{tabular}{|c|c|c|c|c|c|c|c|c|c|c|c|c|}
\hline$\eta_{3 C} C$ & $\eta_{4 A}$ & $\eta_{4 B}$ & $\eta_{4 C}$ & $\eta_{4 D}$ & $\eta_{4 E}$ & $\eta_{4 F}$ & $\eta_{4 G}$ & $\eta_{4 H}$ & $\eta_{4 I}$ & $\eta_{4 J}$ & $\eta_{4 K}$ & $\eta_{4 L}$ \\
\hline$(h \odot \odot)$ & & $(h \odot \odot)$ & & & & & & & & & & \\
\hline$(h \bar{h} \odot)$ & & & & & & & & & & & $(h \odot \odot)$ & \\
\hline$(\odot \mathrm{h} \odot)$ & & & & & $(h \odot \odot)$ & & & & & & & \\
\hline
\end{tabular}

\begin{tabular}{|c|c|c|c|c|c|c|c|c|c|c|c|c|}
\hline$\eta_{30}$ & $7_{4 A}$ & $\eta_{4 B}$ & $\eta_{4 c}$ & $\eta_{4 D}$ & $\eta_{4 E}$ & $\eta_{4 \mathrm{~F}}$ & $\eta_{4 G}$ & $\eta_{44}$ & $\eta_{4 I}$ & $\eta_{4 \mathrm{~J}}$ & $\eta_{4 k}$ & $\eta_{4 L}$ \\
\hline$\left(\begin{array}{lll}h & 0 & \theta\end{array}\right)$ & & & $\left.\begin{array}{lll}(\bar{h} & 0 & 0\end{array}\right)$ & & & & & & & & & \\
\hline$(h \bar{h} \theta)$ & & & & & & & & & $\left(\begin{array}{lll}h & 0 & 0\end{array}\right)$ & & & \\
\hline$\left(\begin{array}{lll}(\theta & h & \theta\end{array}\right)$ & & & & & & $(h \odot \theta)$ & & & & & & \\
\hline
\end{tabular}

\section{$\left(\eta_{3}, \eta_{10}\right)$}

\begin{tabular}{|c|c|c|c|c|c|c|c|c|c|c|}
\hline$\eta_{3 A}$ & $\eta_{10}$ & & \begin{tabular}{ll|}
$710 B$ \\
\end{tabular} & $\eta_{10} c$ & $7_{100}$ & $\eta_{10 E}$ & $\eta_{10 \mathrm{~F}}$ & \multirow{2}{*}{\multicolumn{2}{|c|}{ 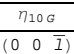 }} & $\eta_{10 H}$ \\
\hline$\left(\begin{array}{llll}0 & 0 & 1\end{array}\right)$ & $\left(\begin{array}{ll}\theta & 0 \\
\end{array}\right.$ & & & & & & & & & \\
\hline$\eta_{38}$ & $\eta_{10 A}^{A}$ & $\eta_{10 \mathrm{~B}}$ & $\eta_{100}$ & & 1100 & $\overline{\eta_{10 \mathrm{E}}}$ & $\bar{\eta}_{1}$ & & $\overline{7100}$ & $\eta_{10 \mathrm{H}}$ \\
\hline$\left(\begin{array}{lll}0 & 0 & 1\end{array}\right)$ & & & & & $\begin{array}{lll}0 & 1)\end{array}$ & & & $\bar{I})$ & & \\
\hline$\eta_{33}$ & $\overline{710 A}$ & $\eta_{10}$ & & $\eta_{10 c}$ & $\eta_{1100}$ & $\bar{\eta}_{11}$ & & $\eta_{10 F}$ & $\eta_{106}$ & $\mathrm{r}_{10 \mathrm{H}}$ \\
\hline$\left(\begin{array}{lll}(0 & 0 & 1\end{array}\right)$ & & $\begin{array}{lll}0 & 0\end{array}$ & $\bar{I}$ & & & $(\theta)$ & 1) & & & \\
\hline$\eta_{30}$ & $\eta_{10 \mathrm{~A}}$ & $\eta_{10 B}$ & $\eta_{1}$ & $10 \mathrm{Co}$ & 7100 & $\eta_{10 \mathrm{E}}$ & $\eta_{10 \mathrm{~F}}$ & $\eta_{10} \mathrm{c}$ & & $70 \mathrm{H}$ \\
\hline$\left(\begin{array}{lll}0 & 0 & 1\end{array}\right)$ & & & 10 & $\begin{array}{ll}\theta \overline{1} \\
\end{array}$ & & & & & & $\begin{array}{lll}\theta & 1)\end{array}$ \\
\hline
\end{tabular}


$\left(\eta_{4}, \eta_{1}\right)$

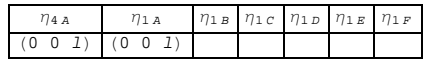

\begin{tabular}{|c|c|c|c|c|c|c|}
\hline$\eta_{4 B}$ & $\eta_{1 A}$ & $\eta_{1 B}$ & $\eta_{1 C}$ & $\eta_{10}$ & $\eta_{1 E}$ & $\eta_{1 F}$ \\
\hline$(\Theta \odot 1)$ & $(\odot \odot \odot)$ & & & & & \\
\hline
\end{tabular}

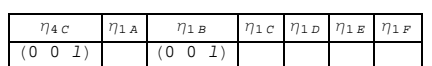

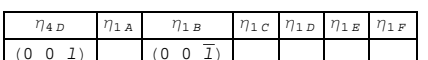
\begin{tabular}{|c|c|c|c|c|c|c|}
\hline$\eta_{4 E}$ & $\eta_{1 A}$ & $\eta_{1 B}$ & $\eta_{1 C}$ & $\eta_{10}$ & $\eta_{1 E}$ & $\eta_{1 F}$ \\
\hline$(\Theta \odot 1)$ & & & $(\Theta \odot \Theta)$ & & & \\
\hline
\end{tabular} \begin{tabular}{|c|c|c|c|c|c|c|}
\hline$\eta_{4 F}$ & $\eta_{1 A}$ & $\eta_{1 B}$ & $\eta_{1 C}$ & $\eta_{10}$ & $\eta_{1 E}$ & $\eta_{1 F}$ \\
\hline$(\theta \theta$ & & & & \\
\hline
\end{tabular} \begin{tabular}{|c|c|c|c|c|c|c|}
\hline$\eta_{4 G}$ & $\eta_{1 A}$ & $\eta_{18}$ & $\eta_{1 C}$ & $\eta_{10}$ & $\eta_{1 E}$ & $\eta_{1 F}$ \\
\hline$(0 \odot 1)$ & & & & $(\Theta \odot 1)$ & & \\
\hline
\end{tabular}

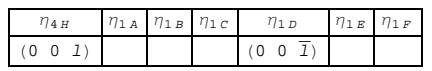
\begin{tabular}{|c|c|c|c|c|c|c|}
\hline$\eta_{4 I}$ & $\eta_{1 A}$ & $\eta_{18} B$ & $\eta_{1 C}$ & $\eta_{10}$ & $\eta_{1 E}$ & $\eta_{1 F}$ \\
\hline$(001)$ & & & & & $(0 \Theta 1)$ & \\
\hline
\end{tabular}

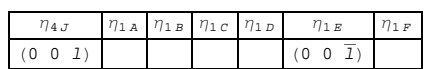

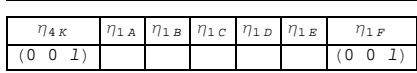

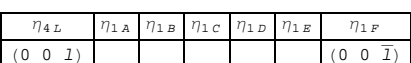

$\left(\eta_{4}, \eta_{3}\right)$

\begin{tabular}{|c|c|c|c|c|c|}
\hline$\eta_{4 A}$ & $\eta_{3}$ & & $\begin{array}{lll}\eta_{38} & 7 \\
\end{array}$ & $\eta_{3 c}$ & 730 \\
\hline$(h \quad \theta \quad \theta)$ & $\overline{l \hbar h}$ & & & & \\
\hline$\eta_{4 B}$ & $\eta_{34}$ & $\eta_{38}$ & $\eta_{30}$ & & $\eta_{30}$ \\
\hline $\left.\begin{array}{lll}h & 0 & 0\end{array}\right)$ & & & (h) & (ब) & \\
\hline$\eta_{4 c}$ & $\eta_{3}$ & $\eta_{38}$ & $\eta_{3 c}$ & & 10 \\
\hline $\left.\begin{array}{llll}h & 0 & \theta\end{array}\right)$ & & & & $\langle\overline{\langle\hbar 0}$ & 0 (1) \\
\hline$n_{40}$ & $\eta_{34}$ & $\eta_{3}$ & & $\eta_{30}$ & $n_{30}$ \\
\hline$(h \odot \theta)$ & & $(\bar{h} h$ & (0) & & \\
\hline$\frac{\eta_{4 E}}{(h \circ \theta \theta)}$ & $\eta_{3 A}$ & \begin{tabular}{|l|l|}
738 \\
\end{tabular} & $\begin{array}{ll}\eta_{33} \\
(\theta h h\end{array}$ & $\frac{c}{c}$ & 730 \\
\hline$\frac{\eta_{4 F}}{(h \theta 0)}$ & \begin{tabular}{ll|} 
\\
\end{tabular} & \begin{tabular}{|l|}
$7_{38}$ \\
\end{tabular} & \begin{tabular}{ll|}
$n_{3 c}$ \\
\end{tabular} & & $\frac{1}{10}$ \\
\hline$\eta_{4 G}$ & $\overline{n_{3}}$ & & $\eta_{33}$ & $\eta_{3 c}$ & $\eta_{30}$ \\
\hline 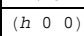 & $\frac{1 \theta h}{(\theta h}$ & (0) & & & \\
\hline$\eta_{4 \mathrm{H}}$ & $\eta_{3 A}$ & $\eta_{3}$ & & $\eta_{3 c}$ & $\eta_{30}$ \\
\hline$(h \circ \theta 0)$ & & $(\theta)$ & 70) & & \\
\hline$\eta_{4 I}$ & \begin{tabular}{|l|}
$\eta_{3 A}$ \\
\end{tabular} & $\eta_{38}$ & $\eta_{3 c}$ & & \\
\hline $\left.\begin{array}{lll}(h & 0 & \theta\end{array}\right)$ & & & & $\langle\overline{(h \bar{h}}$ & (8) \\
\hline $\begin{array}{l}\eta_{4 j} \\
(h \circ 00)\end{array}$ & $\begin{array}{r}\eta_{3} \\
h \text { (h) }\end{array}$ & 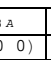 & \begin{tabular}{|l|}
$7_{38}$ \\
\end{tabular} & $\eta_{3 c}$ & $n_{30}$ \\
\hline$\eta_{4 k}$ & $\eta_{3 A}$ & $\eta_{3 B}$ & $\eta_{3}$ & & $\eta_{30}$ \\
\hline $\begin{array}{ll}(h \odot \theta) & \theta\end{array}$ & & & & & \\
\hline$\frac{\eta_{4 L}}{(h 00)}$ & \begin{tabular}{|l|}
$n_{3 A}$ \\
\end{tabular} & $\begin{array}{r}\eta_{3} \\
h_{3}\end{array}$ & \begin{tabular}{|l|l|}
$B$ & \\
& \\
\end{tabular} & $\eta_{3 c}$ & $\eta_{30}$ \\
\hline$(h \odot \theta)$ & & & & & \\
\hline
\end{tabular}


$\left(\eta_{4}, \eta_{5}\right)$

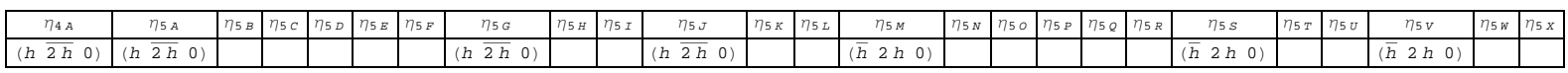

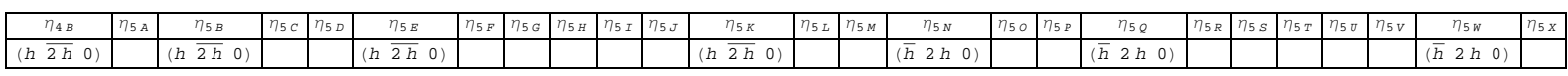

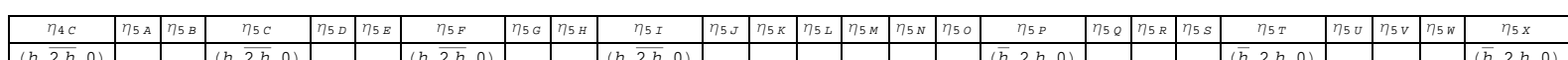

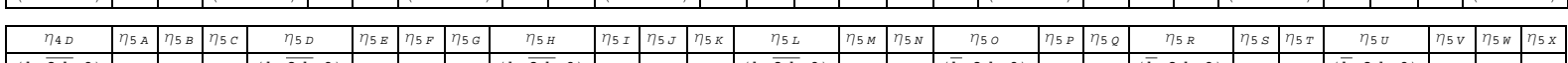

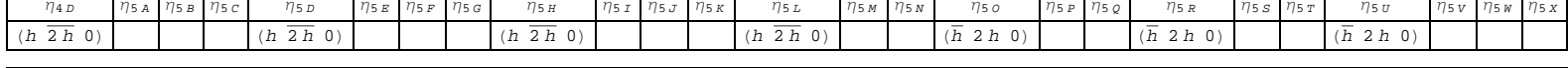

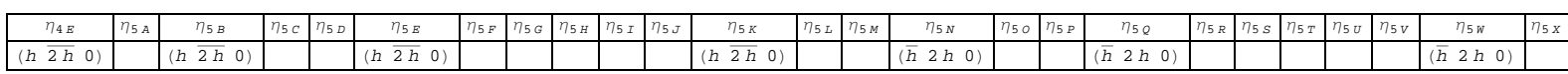

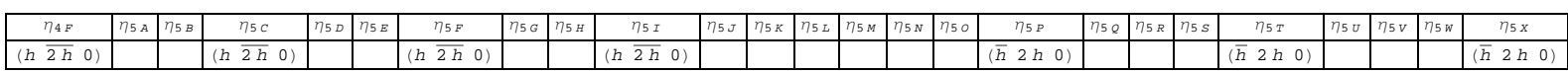

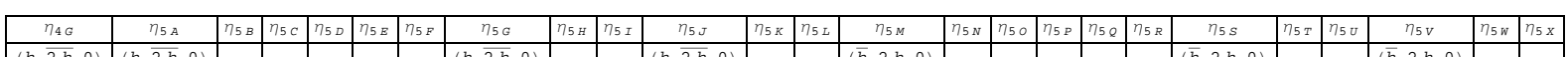

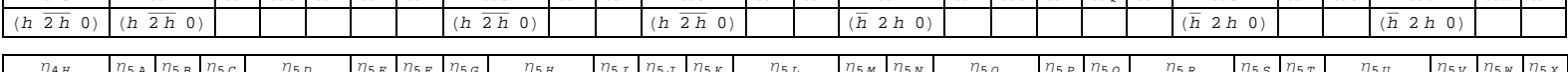

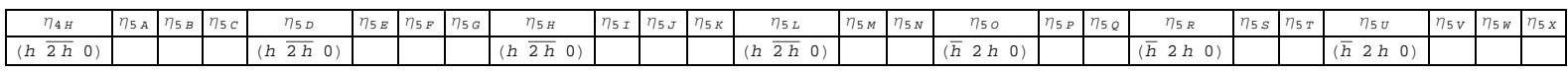

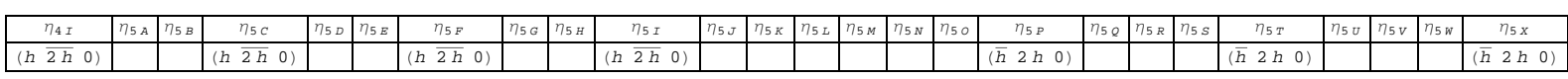

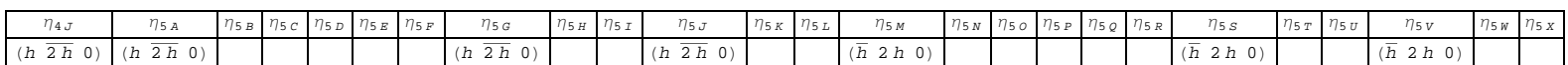

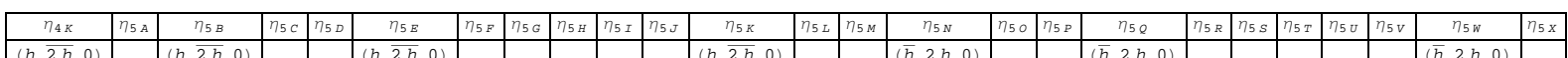

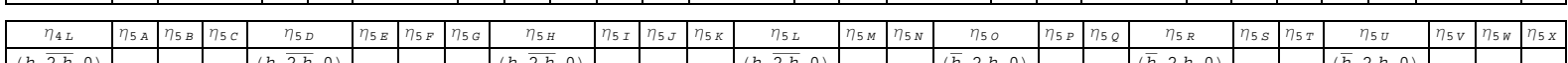

$\left(\eta_{4}, \eta_{6}\right)$

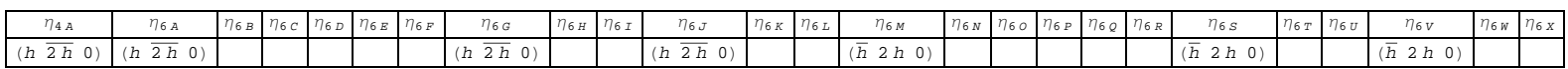

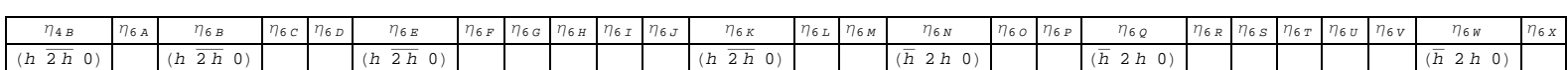

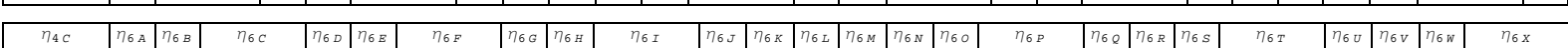

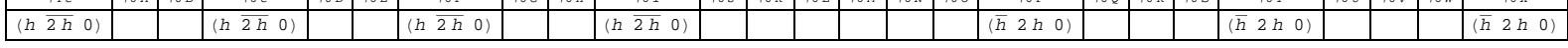

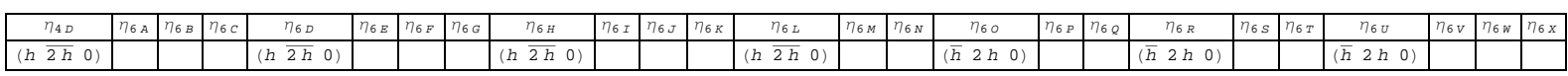

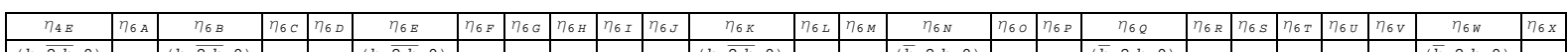

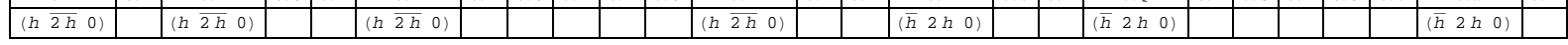

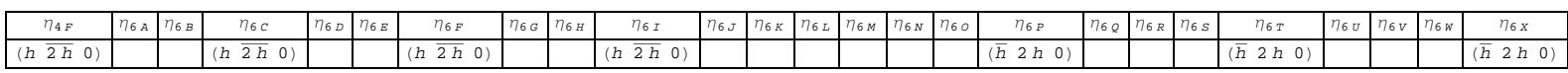

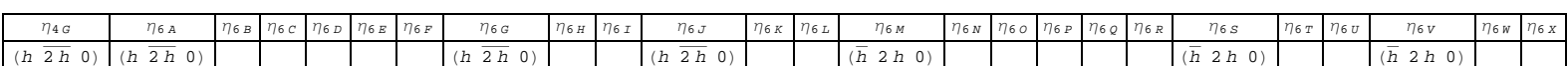

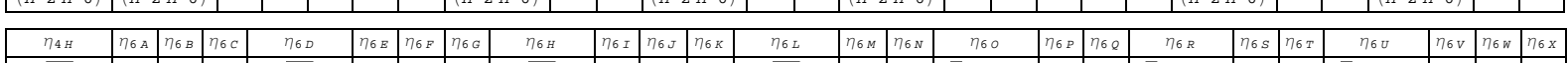

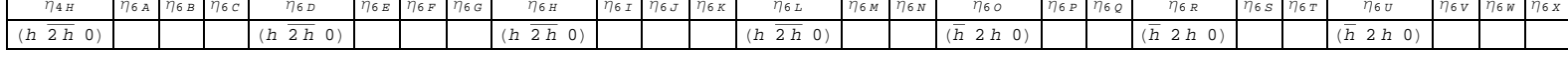

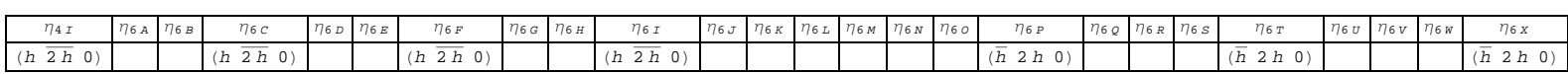

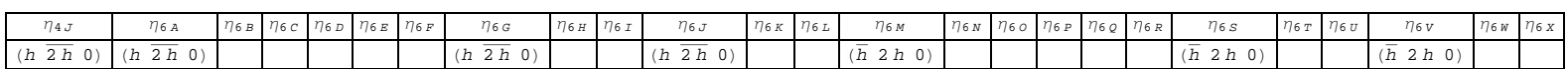

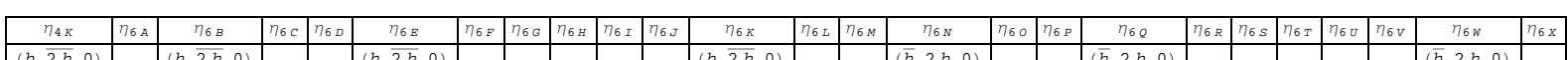

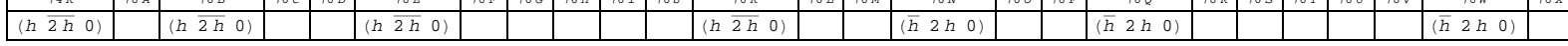

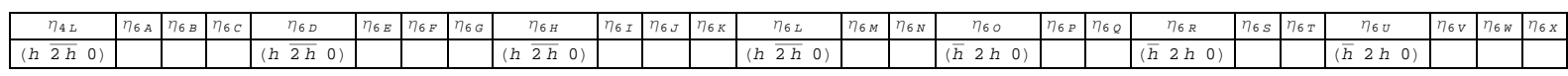


$\left(\eta_{4}, \eta_{7}\right)$

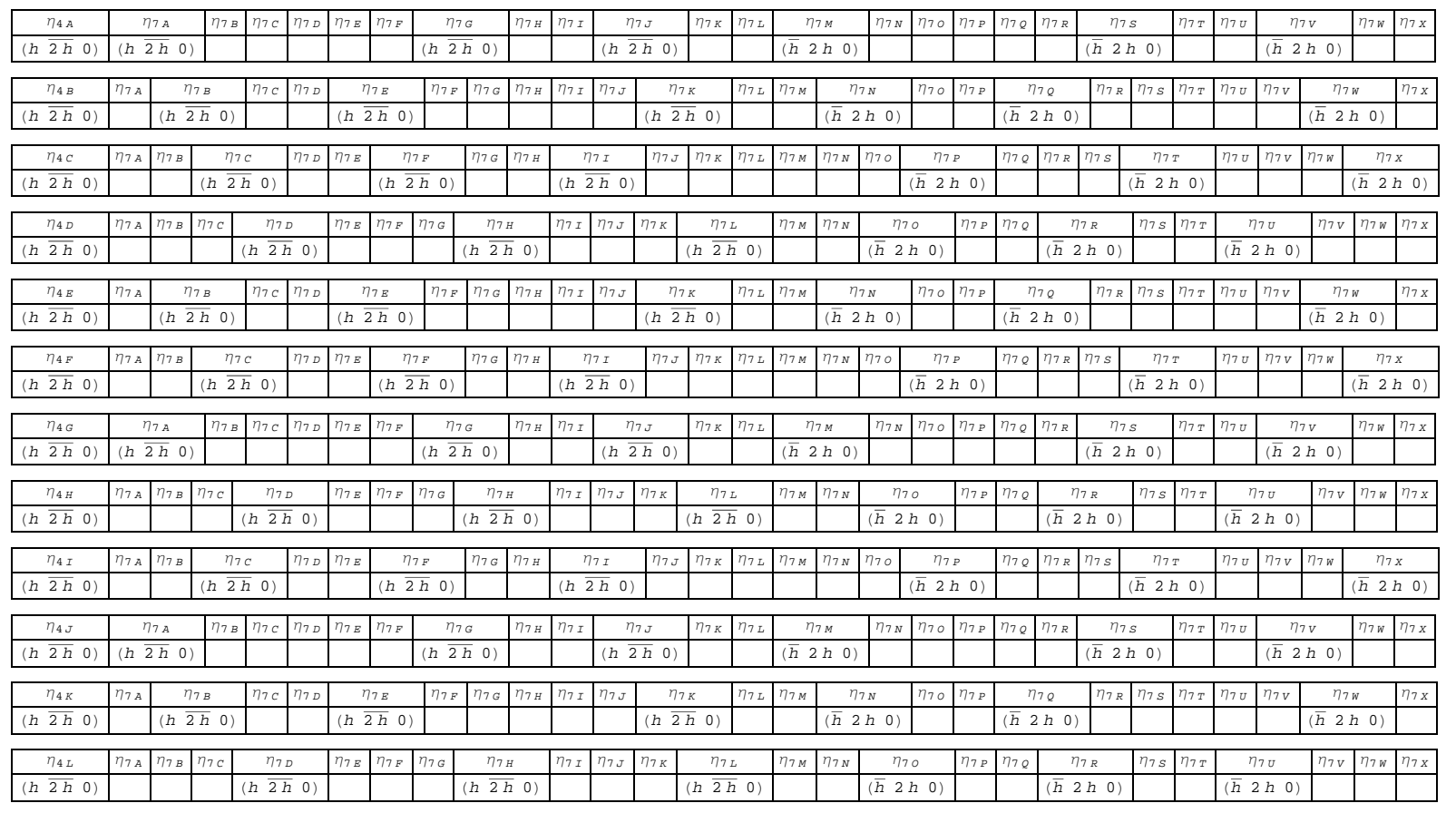


$\left(\eta_{4}, \eta_{9}\right)$

\begin{tabular}{|c|c|c|c|c|c|c|c|}
\hline$\eta_{4 A}$ & \multicolumn{2}{|c|}{$\frac{\eta_{9 A}}{(\theta \Theta \theta 1)}$} & $\eta_{98}$ & $\eta_{9 c}$ & $\eta_{90}$ & $\eta_{9 E}$ & $\eta_{9 F}$ \\
\hline $7_{4 B}$ & $\eta_{9}$ & & $\overline{\eta_{9 B}}$ & $\eta_{9 c}$ & \begin{tabular}{ll|}
790 \\
\end{tabular} & 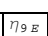 & $\eta_{9 F}$ \\
\hline$\left(\begin{array}{llll}0 & 0\end{array}\right)$ & $\langle\theta 0$ & $\overline{\mid} \bar{I})$ & & & & & \\
\hline$\eta_{4 C}$ & $\eta_{9 A}$ & $\eta_{s}$ & & $\eta_{9 c}$ & $\eta_{90}$ & $\eta_{9 E}$ & $\eta_{9 F}$ \\
\hline$\left(\begin{array}{llll}0 & 1\end{array}\right)$ & & $10 \mathrm{C}$ & 1) & & & & \\
\hline$\eta_{4 D}$ & $\eta_{9 A}$ & & & $\eta_{9 c}$ & $\eta_{90}$ & $\eta_{9 E}$ & $\eta_{9 F}$ \\
\hline$\left(\begin{array}{lll}\left(\begin{array}{lll}0 & 1\end{array}\right)\end{array}\right.$ & & $(\theta)$ & $\bar{I})$ & & & & \\
\hline$\frac{\eta_{4 E}}{(\theta 0 \theta 1)}$ & $\eta_{9 A}$ & $\eta_{98}$ & $\frac{\eta_{3}}{1 \theta}$ & & $\eta_{90}$ & $\eta_{9 E}$ & $\eta_{9 F}$ \\
\hline$\eta_{4 \mathrm{~F}}$ & $\eta_{9 A}$ & $\eta_{98}$ & $\eta_{\xi}$ & & $\eta_{90}$ & $\bar{\eta}_{{ }_{E}}$ & $\eta_{9 F}$ \\
\hline$\left(\begin{array}{lll}0 & 0 & 1\end{array}\right)$ & & & $(\theta)$ & $\bar{I})$ & & & \\
\hline$\eta_{46}$ & $\eta_{9 \mathrm{~A}}$ & $\eta_{98}$ & $\eta_{9 c}$ & & & $\eta_{9 E}$ & $\eta_{9 F}$ \\
\hline$\left(\begin{array}{lll}\left(\begin{array}{lll}0 & 1\end{array}\right)\end{array}\right.$ & & & & $(\theta)$ & 1) & & \\
\hline$\eta_{44}$ & $\eta_{9 A}$ & 798 & $\eta_{9 c} \mathrm{c}$ & & & $\eta_{9 E}$ & $\eta_{9 F}$ \\
\hline$\left(\begin{array}{lll}(0 & 0 & 1\end{array}\right)$ & & & & & $\bar{I} \overline{1}$ & & \\
\hline$\frac{\eta_{4 I}}{(001)}$ & $\eta_{9 A}$ & $\eta_{9 B}$ & $\eta_{9 c}$ & $7_{90}$ & $\eta_{9}$ & $\frac{E E}{E E}$ & $\eta_{9 F}$ \\
\hline$\eta_{4 \mathrm{~J}}$ & $\eta_{9 A}$ & $79 B$ & $\eta_{9 c}$ & $7_{90}$ & & & $\eta \eta_{9 F}$ \\
\hline$\left(\begin{array}{lll}0 & 0 & 1\end{array}\right)$ & & & & & & $\theta \bar{I}$ & \\
\hline 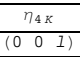 & $7_{9 A}$ & $79 B$ & $\eta_{9 c}$ & $\eta_{90}$ & \begin{tabular}{|l|l|}
$79 E$ \\
\end{tabular} & & b \\
\hline$\eta_{4 L}$ & $\eta_{9 A}$ & $79 B$ & $\eta_{9 c}$ & $\eta_{9 D}$ & $\eta_{9 \mathrm{E}}$ & $\eta_{9}$ & \\
\hline$\left(\begin{array}{lll}0 & 0 & 1\end{array}\right)$ & & & & & & & $\bar{I})$ \\
\hline
\end{tabular}

$\left(\eta_{4}, \eta_{11}\right)$

\begin{tabular}{|c|c|c|c|c|c|c|c|c|c|c|c|c|c|}
\hline$\eta_{4 A}$ & $\eta_{11 \mathrm{~A}}$ & & $\eta_{11 B}$ & $\eta_{11}$ & \begin{tabular}{l|l}
$11 c$ & $\eta_{11}$ \\
\end{tabular} & \begin{tabular}{l|l}
110 & $\eta_{1}$ \\
\end{tabular} & \begin{tabular}{l|l}
$\eta_{111}$ & $\eta_{1}$
\end{tabular} & \begin{tabular}{ll|l}
$\eta_{111}$ & $\eta_{1}$ \\
\end{tabular} & \begin{tabular}{ll|l}
$\eta_{116}$ & $\eta_{11}$
\end{tabular} & $\eta_{111 \mathrm{H}} \mid \eta_{1}$ & \begin{tabular}{l|l}
1111 & $\eta_{11 \mathrm{~J}}$ \\
\end{tabular} & $\eta_{111}$ & $\eta_{11 L}$ \\
\hline $\begin{array}{lll}(0 & 0 & 1)\end{array}$ & (e) 0 & 1) & 001 & & & & & & & & & & \\
\hline$\eta_{4 B}$ & $\eta_{111}$ & & $\eta_{118}$ & $\eta_{11}$ & \begin{tabular}{l|l}
$11 c$ & $\eta_{11}$ \\
\end{tabular} & \begin{tabular}{l|l}
1110 & $\eta_{1}$
\end{tabular} & $\begin{array}{ll}\eta_{111 \mathrm{E}} & \eta_{1}\end{array}$ & \begin{tabular}{ll|l}
$\eta_{111}$ & $\eta_{1}$
\end{tabular} & $\begin{array}{ll}\eta_{116} & \eta_{11}\end{array}$ & $711 \mathrm{H}$ & 111 & 111 & $\eta_{11 L}$ \\
\hline $\begin{array}{llll}0 & 0 & 1)\end{array}$ & $\left(\begin{array}{lll}\theta & 0 \\
\end{array}\right.$ & & $\begin{array}{lll}0 & 0 & 1\end{array}$ & & & & & & & & & & \\
\hline$\eta_{4 c}$ & $\eta_{11 \mathrm{~A}}$ & $\eta_{11 B}$ & $\eta_{11}$ & & $\eta_{11 D}$ & & \begin{tabular}{l|l}
$\eta_{111}$ & $\eta_{1}$
\end{tabular} & 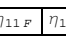 & 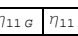 & \begin{tabular}{ll|l}
$\eta_{111}$ & $\eta_{1}$
\end{tabular} & \begin{tabular}{l|l}
1111 & $\eta_{11 \mathrm{~J}}$ \\
\end{tabular} & $\eta_{111}$ & $\eta_{11 L}$ \\
\hline $\begin{array}{llll}0 & 0 & 11 \\
\end{array}$ & & & $(\theta)$ & & $\left(\begin{array}{llll}0 & 01\end{array}\right.$ & & & & & & & & \\
\hline 740 & $\eta_{11 \mathrm{~A}}$ & $\eta_{11 B}$ & $\eta_{11}$ & & $\eta_{110}$ & & $\eta_{111 E} \mid \eta_{1}$ & $\begin{array}{lll}\eta_{11} & \eta_{1}\end{array}$ & $\eta_{116} \mid \eta_{11}$ & \begin{tabular}{ll|l}
$n_{11 H}$ & $\eta_{1}$
\end{tabular} & $\bar{\eta}_{11 \mathrm{~J}}$ & $\eta_{111}$ & $\eta_{11 L}$ \\
\hline $\begin{array}{llll}0 & 0 & 1\end{array}$ & & & $\langle\theta$ & & $\begin{array}{llll}0 & \theta & 1\end{array}$ & & & & & & & & \\
\hline $74 E$ & \begin{tabular}{|l|}
$\eta_{11 A}$ \\
\end{tabular} & $\eta_{11 B}$ & $\eta_{11 c}$ & $\eta_{110}$ & $\eta_{11}$ & $11 E$ & $\eta_{11 f}$ & & \begin{tabular}{l|l|}
$\eta_{116}$ & $\eta_{11}$
\end{tabular} & \begin{tabular}{l|l}
$11 \mathrm{H}$ & $\eta_{1}$
\end{tabular} & $\eta_{11 \mathrm{~J}}$ & $\eta_{11 !}$ & $\eta_{11 L}$ \\
\hline$\left(\begin{array}{lll}(0 & \theta & 1\end{array}\right)$ & & & & & & \begin{tabular}{ll|}
$01)$ \\
\end{tabular} & $\begin{array}{llll} & 0 & 0 \\
\end{array}$ & $\overline{1}$ & & & & & \\
\hline$\eta_{4 F}$ & $\eta_{11 \mathrm{~A}}$ & $\eta_{11 B}$ & $\eta_{11 c}$ & $\eta_{110}$ & & $11 E$ & $\eta_{111}$ & & 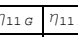 & \begin{tabular}{l|l}
$11 H$ & $\eta_{1}$
\end{tabular} & \begin{tabular}{l|l}
111 & $\eta_{11 \mathrm{~J}}$
\end{tabular} & $\eta_{111}$ & $\eta_{11 L}$ \\
\hline$\left(\begin{array}{lll}0 & 0 & 1\end{array}\right)$ & & & & & $\left(\begin{array}{ll}0 \\
\end{array}\right.$ & \begin{tabular}{ll|l}
$\theta$ & $\overline{1})$ \\
\end{tabular} & $\left(\begin{array}{ll}0 & \theta \\
\end{array}\right.$ & 1) & & & & & \\
\hline$\eta_{46}$ & \begin{tabular}{|l|}
$\eta_{11 A}$ \\
\end{tabular} & $\eta_{11 B}$ & $\eta_{11 c}$ & $\eta_{110}$ & $\eta_{11 E}$ & \begin{tabular}{|l|l}
$E$ & $\eta_{11 F}$
\end{tabular} & & 1116 & $\eta_{11 H}$ & & \begin{tabular}{l|l}
1111 & $\eta_{11 \mathrm{~J}}$ \\
\end{tabular} & $\eta_{111}$ & $n_{11 L}$ \\
\hline$\left(\begin{array}{lll}\left(\begin{array}{lll}0 & 1\end{array}\right)\end{array}\right.$ & & & & & & & & $\begin{array}{lll} & 1\end{array}$ & $(\theta \odot \bar{I}$ & $\overline{\bar{I}})$ & & & \\
\hline$\eta_{44}$ & $\eta_{11 \mathrm{~A}}$ & $\eta_{11 B}$ & $\eta_{11 c}$ & $\eta_{110}$ & \begin{tabular}{l|l}
0 & $\eta_{111}$
\end{tabular} & \begin{tabular}{|l|l|}
$E$ & $\eta_{11 F}$
\end{tabular} & & $\frac{1116}{116}$ & $\eta_{11 \mathrm{H}}$ & & \begin{tabular}{l|l}
111 & $\eta_{11} J$
\end{tabular} & $\eta_{11 !}$ & $\eta_{11 L}$ \\
\hline$\left(\begin{array}{lll}0 & 0 & 1\end{array}\right)$ & & & & & & & & $\begin{array}{ll}\theta & 1\end{array}$ & 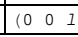 & & & & \\
\hline$\eta_{4 I}$ & \begin{tabular}{|l|}
$\eta_{11 \mathrm{~A}}$ \\
\end{tabular} & $\eta_{11 B}$ & $\eta_{11 c}$ & $\eta_{110}$ & $\eta_{11 E}$ & \begin{tabular}{l|l}
$E$ & $\eta_{11 F}$
\end{tabular} & \begin{tabular}{l|l}
$F$ & $\eta_{116}$
\end{tabular} & \begin{tabular}{l|l}
$G$ & $\eta_{11 H}$
\end{tabular} & $\eta_{11}$ & $11 \mathrm{I}$ & $\eta_{11 \mathrm{~J}}$ & $\eta_{11 !}$ & $\mid \eta_{11 L}$ \\
\hline 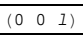 & & & & & & & & & \begin{tabular}{l|ll} 
& $<\theta$ & $\theta$ \\
\end{tabular} & $\begin{array}{lll}0 & 1)\end{array}$ & $\left(\begin{array}{lll}0 & 0 & 1\end{array}\right)$ & & \\
\hline$\eta_{4 \mathrm{~J}}$ & \begin{tabular}{|l|l|}
$\eta_{11 A}$ \\
\end{tabular} & $\eta_{11 B}$ & $\eta_{11 c}$ & $\eta_{110}$ & \begin{tabular}{|l|l|}
$\eta_{111}$ \\
\end{tabular} & \begin{tabular}{l|l}
$E$ & $\eta_{11 F}$
\end{tabular} & $\eta_{11 G}$ & \begin{tabular}{l|l}
6 & $\eta_{11 H}$
\end{tabular} & $\eta_{11}$ & 111 & $\eta_{11 \mathrm{~J}}$ & 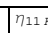 & $\mid n_{11 L}$ \\
\hline$\left(\begin{array}{lll}(0 & 0 & 1\end{array}\right)$ & & & & & & & & & $(\theta)$ & \begin{tabular}{ll|}
$\theta \overline{1})$ \\
\end{tabular} & $\left(\begin{array}{lll}0 & 0 & 1\end{array}\right)$ & & \\
\hline$\eta_{4 k}$ & \begin{tabular}{|l|l|}
$\eta_{11 A}$ & \\
\end{tabular} & $\eta_{11 B}$ & $\eta_{11 c}$ & $\eta_{110}$ & $\begin{array}{l}\eta_{11 E} \\
\end{array}$ & \begin{tabular}{l|l}
$E$ & $\eta_{11 F}$
\end{tabular} & \begin{tabular}{l|l}
$F$ & $\eta_{116}$
\end{tabular} & \begin{tabular}{l|l}
$G$ & $\eta_{11 H}$
\end{tabular} & \begin{tabular}{l|l}
$H$ & $\eta_{111}$ \\
\end{tabular} & $\eta_{11 \mathrm{~J}}$ & $\eta_{11 \mathrm{~K}}$ & & $\eta_{11 L}$ \\
\hline$\left(\begin{array}{lll}0 & 0 & 1\end{array}\right)$ & & & & & & & & & & & $\left(\begin{array}{lll}0 & 0 \\
\end{array}\right.$ & & $\theta \bar{I}$ \\
\hline$\eta_{4 L}$ & \begin{tabular}{|l|l|}
$\eta_{11 A}$ & \\
\end{tabular} & $\eta_{11 B}$ & $\eta_{11 c}$ & $\eta_{11 D}$ & \begin{tabular}{|l|l|l}
0 & $\eta_{111}$
\end{tabular} & \begin{tabular}{|l|l}
$E$ & $\eta_{11 F}$
\end{tabular} & \begin{tabular}{l|l}
$F$ & $\eta_{116}$
\end{tabular} & \begin{tabular}{c|l}
$G$ & $\eta_{11 H}$
\end{tabular} & \begin{tabular}{l|l|l|}
$H$ & $\eta_{111}$
\end{tabular} & $\eta_{11 \mathrm{~J}}$ & $\eta_{11 k}$ & & $\bar{\eta}_{11 L}$ \\
\hline $\begin{array}{llll}0 & 0 & 11\end{array}$ & & & & & & & & & & & $\left(\begin{array}{llll}0 & 0 & 1\end{array}\right.$ & & 01 \\
\hline
\end{tabular}


$\left(\eta_{5}, \eta_{4}\right)$

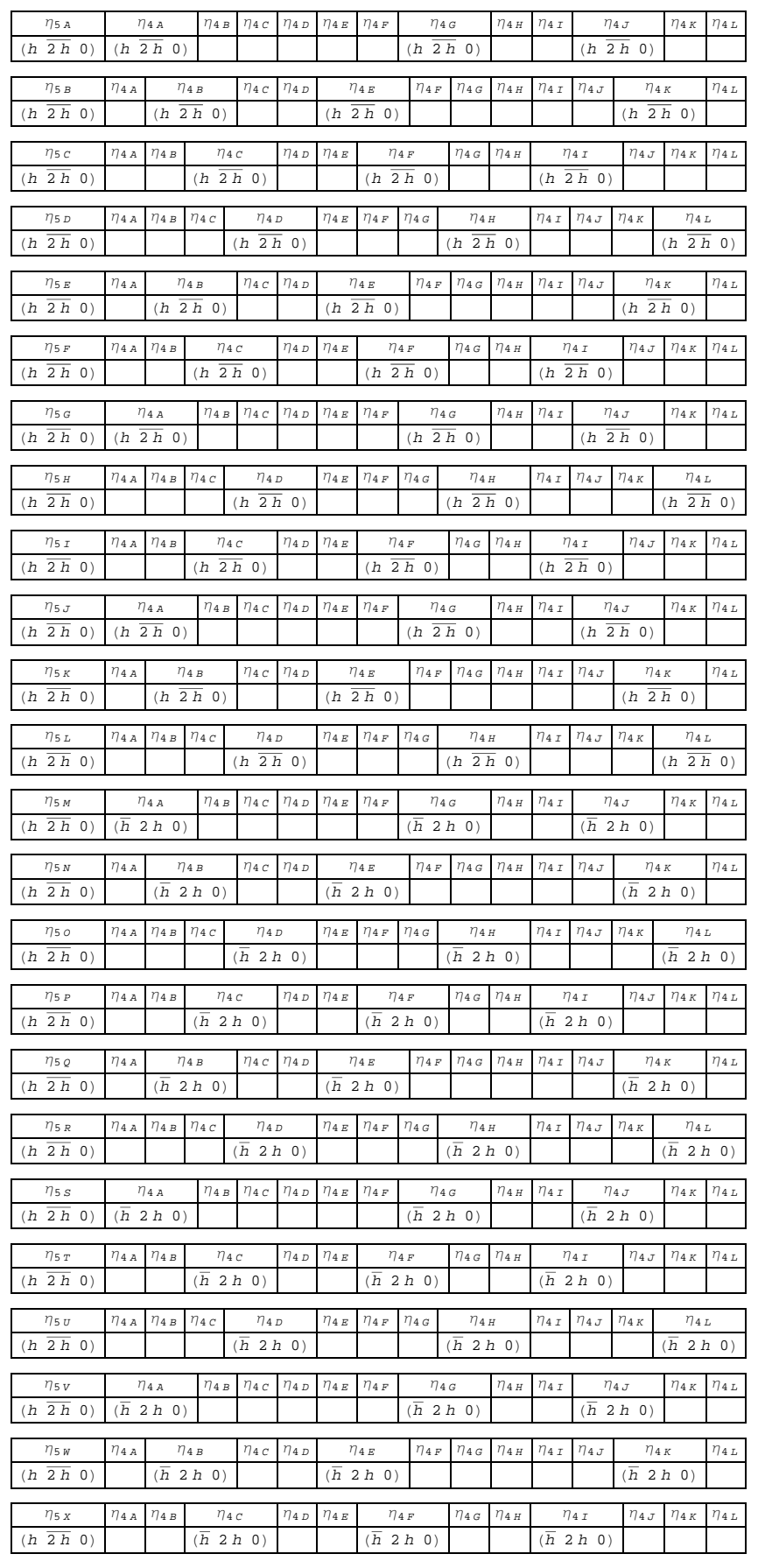


$\left(\eta_{5}, \eta_{6}\right)$

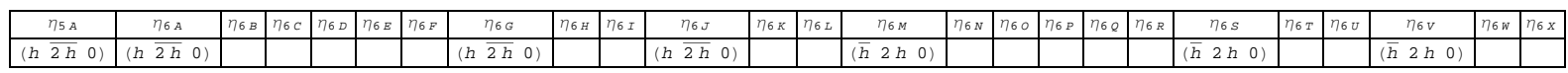

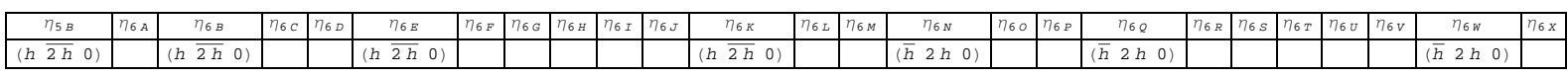

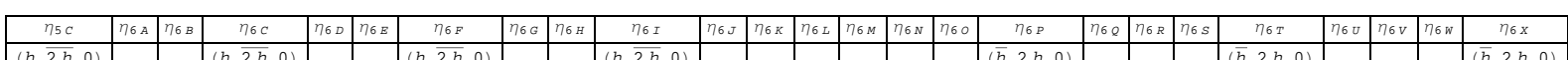

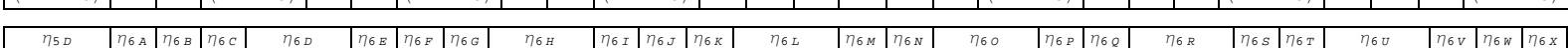

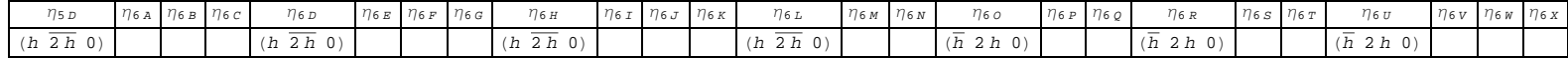

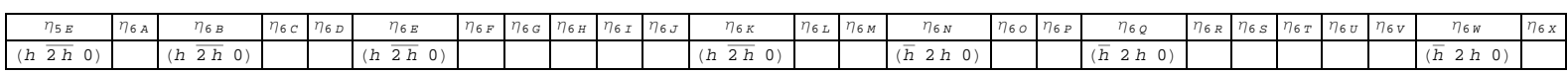

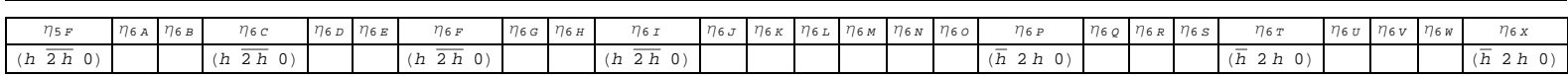

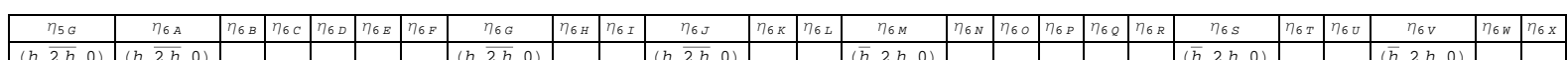

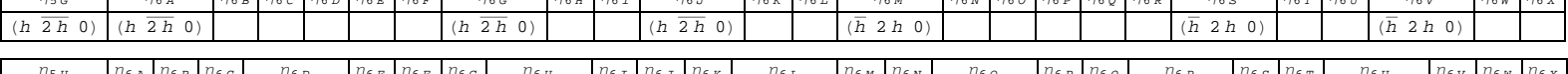

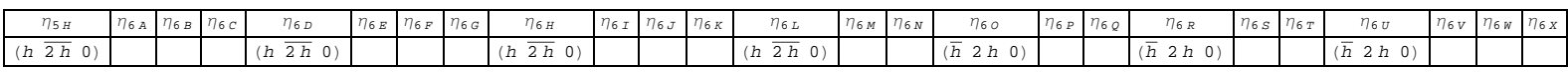

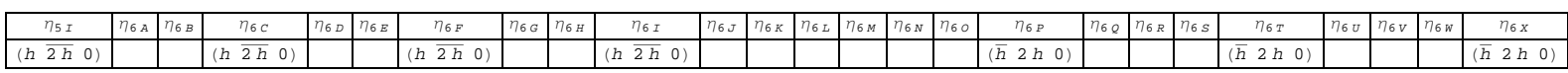

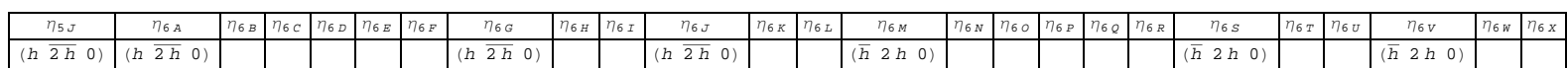

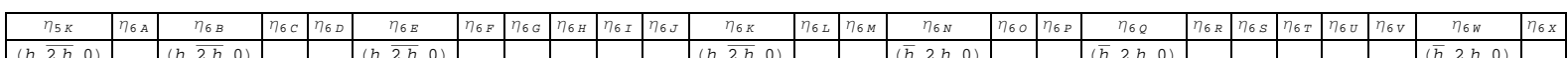

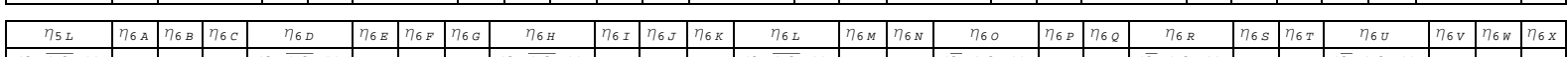

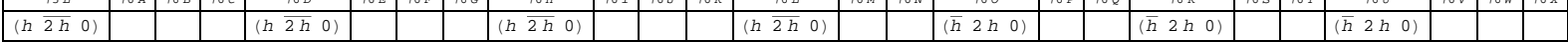

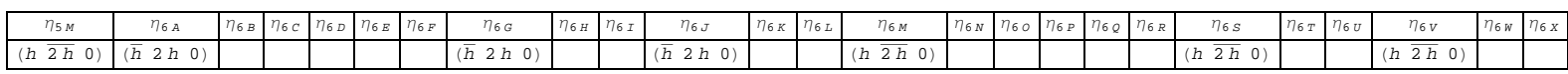

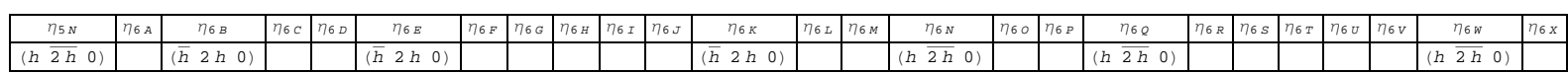

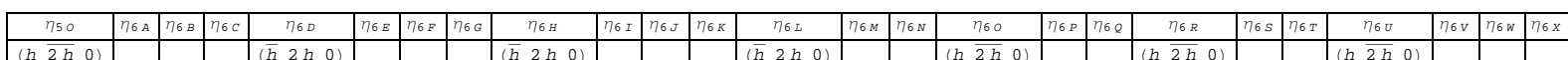

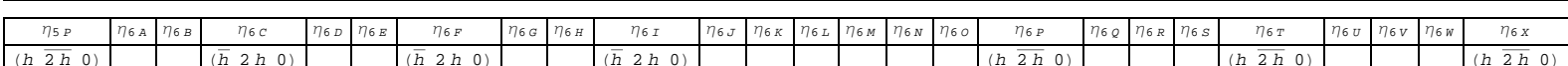

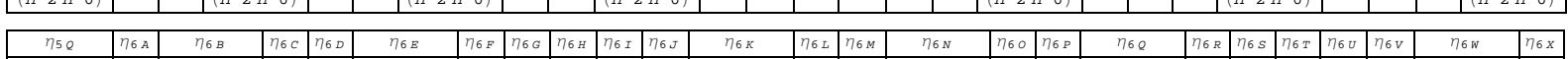

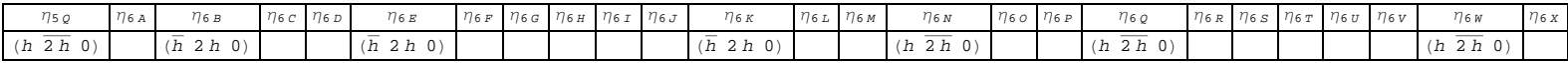

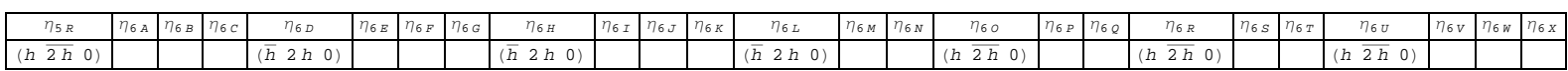

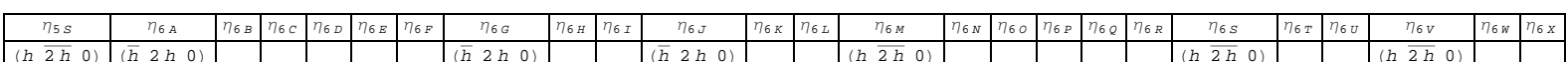

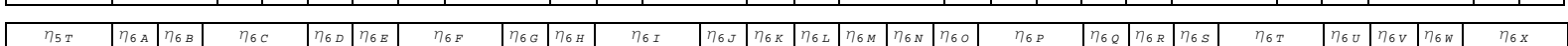

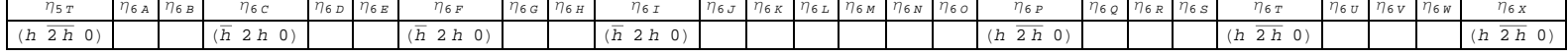

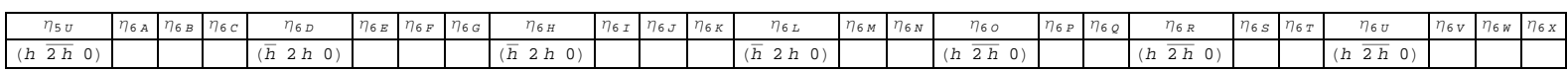

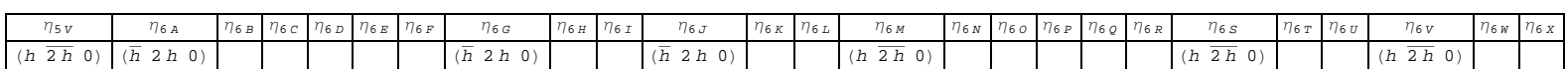

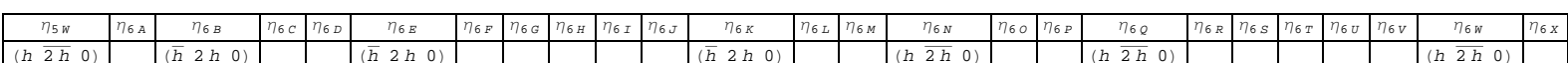

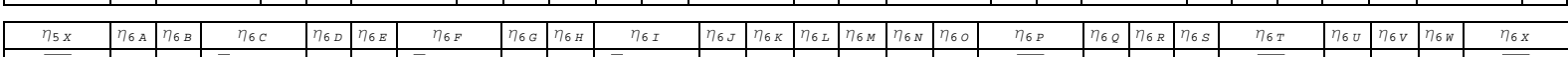

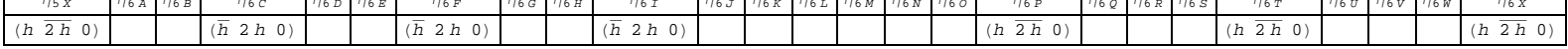


$\left(\eta_{5}, \eta_{7}\right)$

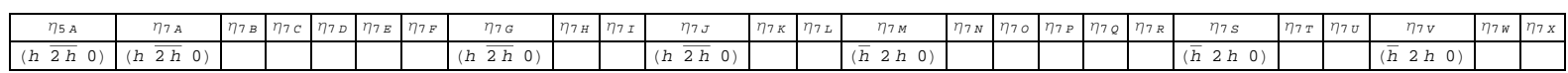

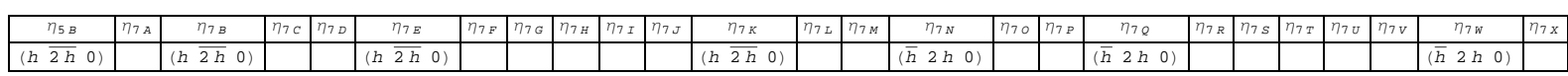

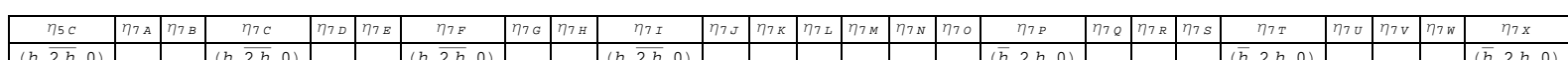

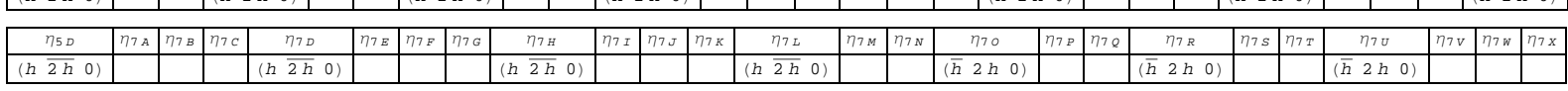

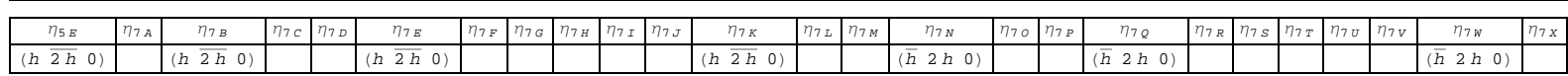

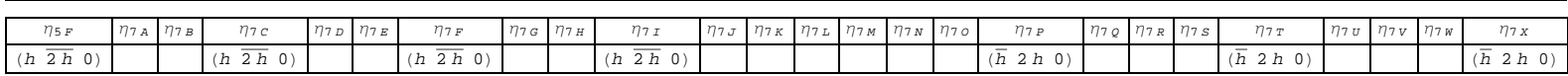
\begin{tabular}{|c|c|c|c|c|c|c|c|c|c|c|c|c|c|c|c|c|c|c|c|c|c|c|c|c|}
\hline$\eta_{56}$ & $\eta_{7 \mathrm{~A}}$ & $\eta_{78}$ & $\eta_{7 \mathrm{C}}$ & $\eta_{70}$ & $\eta_{7 \mathrm{E}}$ & $\eta_{7 \mathrm{~F}}$ & $\eta_{76}$ & $\eta_{7 \mathrm{H}}$ & $\eta_{7 \mathrm{I}}$ & $\eta_{7 J}$ & $\eta_{7 \mathrm{~K}}$ & $\eta_{7 \mathrm{~L}}$ & $\eta_{7 \mathrm{M}}$ & $\eta_{7 \mathrm{~N}}$ & $\eta_{70}$ & $\eta_{7 \mathrm{P}}$ & $\eta_{7 \mathrm{Q}}$ & $\eta_{7 \mathrm{R}}$ & $\eta_{7 \mathrm{~S}}$ & $\eta_{7 \mathrm{~T}}$ & $\eta_{7 \mathrm{U}}$ & $\eta_{7 \mathrm{~V}}$ & $\eta_{7 \mathrm{~W}}$ & $\eta_{7 \mathrm{X}}$ \\
\hline
\end{tabular}

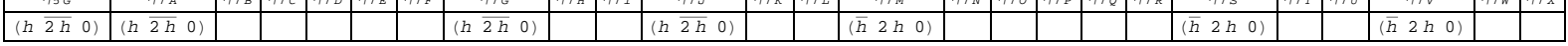

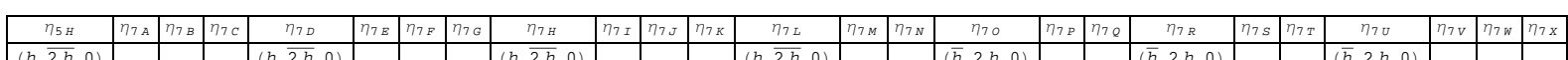

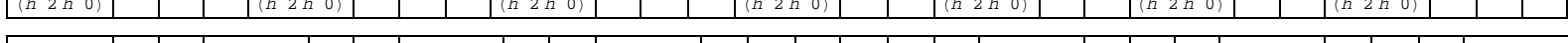

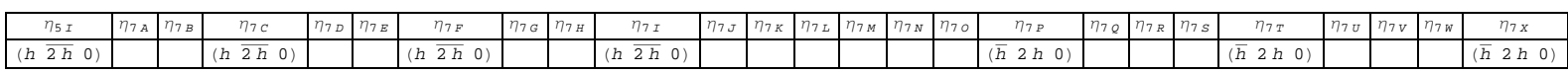

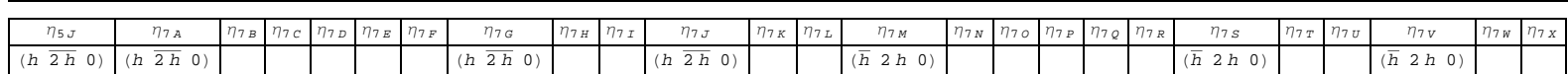

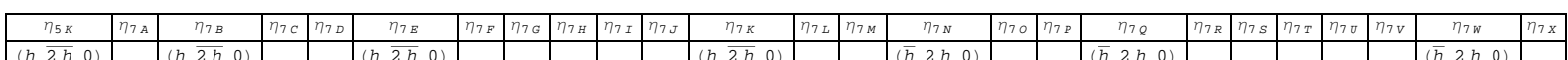

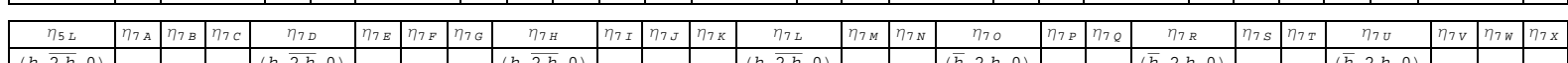

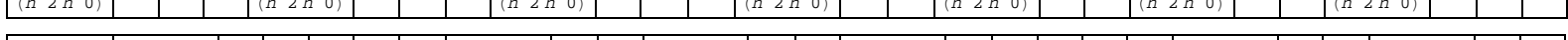

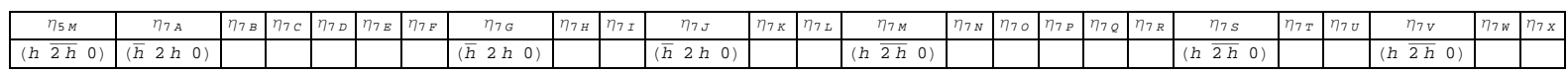

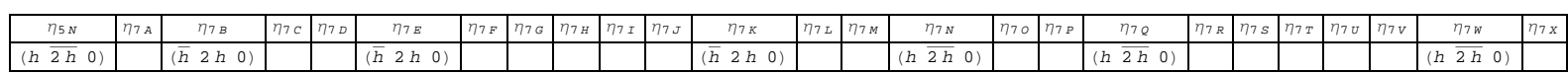

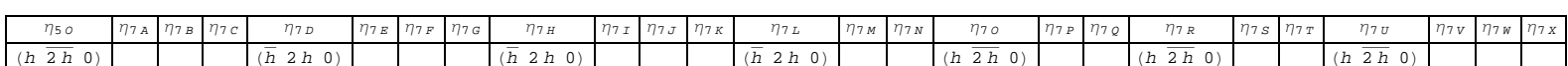

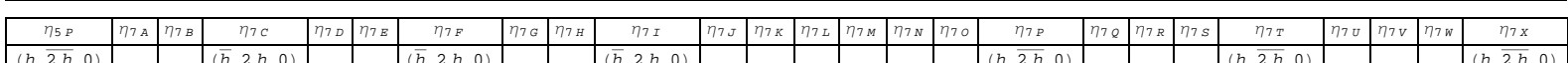

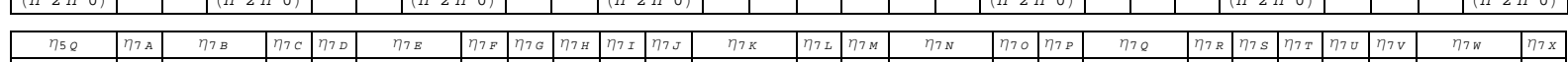

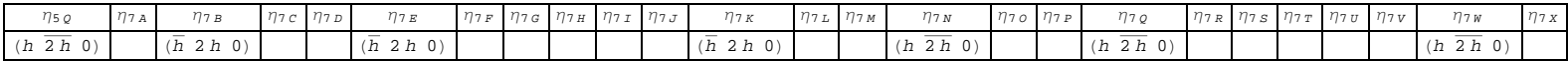

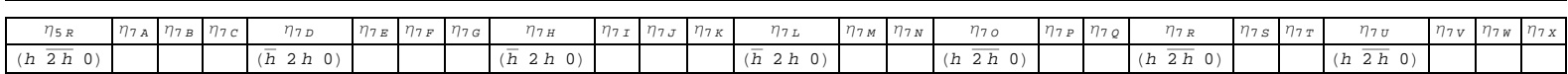

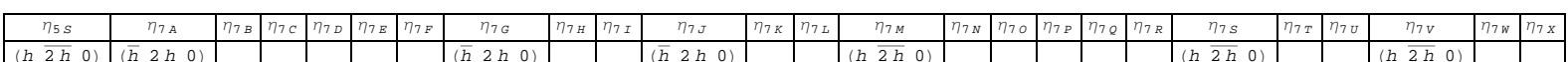

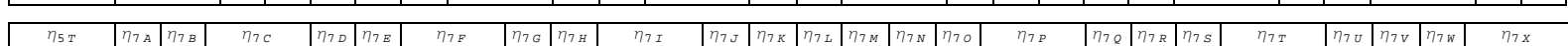

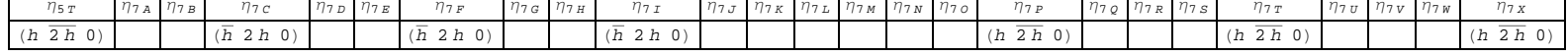

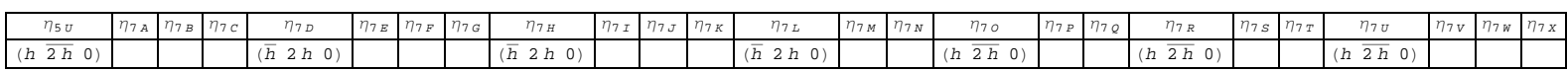

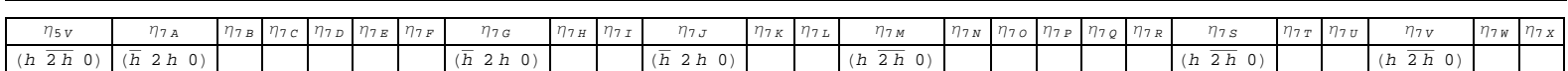

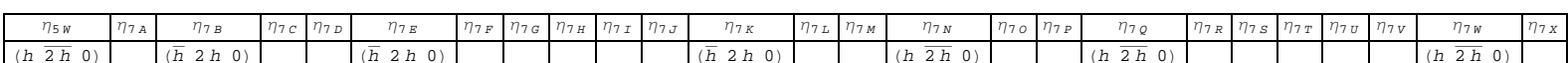

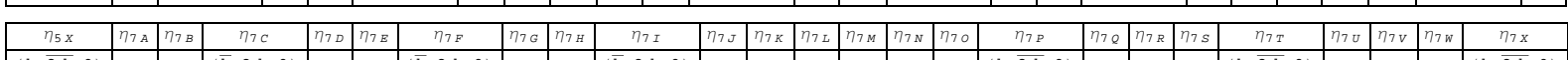

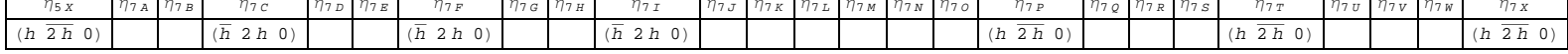


$\left(\eta_{6}, \eta_{4}\right)$

\begin{tabular}{|c|c|c|c|c|c|c|c|c|c|c|c|c|c|c|c|}
\hline$\eta_{6 \mathrm{~A}}$ & & $74 \mathrm{~A}$ & \begin{tabular}{|l|}
$7_{48}$ \\
\end{tabular} & $74 c$ & $\eta_{40}$ & \begin{tabular}{|l|}
$7_{4 E}$ \\
\end{tabular} & $\eta_{4 F}$ & $\eta_{4}$ & & $\eta_{4 \mathrm{H}}$ & $\eta_{4 I}$ & & $74 \mathrm{~J}$ & $\eta_{4 k}$ & $\eta_{4 L}$ \\
\hline$h \overline{2 h} \theta)$ & $(h \overline{2}$ & $2 h \theta)$ & & & & & & & (he) & & & & $2 \hbar$ ब) & & \\
\hline$\eta_{68}$ & $\eta_{4 A}$ & $\eta_{46}$ & & $\eta_{4 c}$ & $\eta_{40}$ & $\eta_{4}$ & & $n_{4 \mathrm{~F}}$ & \begin{tabular}{|l|}
$\eta_{46}$ \\
\end{tabular} & $\eta_{4 \mathrm{H}}$ & $\eta_{4 I}$ & $\eta_{4 \mathrm{~J}}$ & $n_{4}$ & $74 \mathrm{k}$ & $\eta_{4 L}$ \\
\hline$\overline{h \overline{2 h} \Theta)}$ & & $(h \overline{2 h}$ & $\bar{h}$ ब) & & & & $2 \hbar$ ब) & & & & & & & $2 \mathrm{~h} \theta)$ & \\
\hline$\eta_{6 c}$ & $\eta_{4 A}$ & $\eta_{4 B}$ & $\eta_{40}$ & & $\eta_{40}$ & \begin{tabular}{|l|}
$\eta_{4 E}$ \\
\end{tabular} & $\eta_{4}$ & & $\eta_{4 G}$ & $\eta_{4 \mathrm{H}}$ & & 141 & $\eta_{43}$ & \begin{tabular}{l|l}
1 & $\eta_{4 k}$
\end{tabular} & $\eta_{4 L}$ \\
\hline $\begin{array}{l}n \overline{2 h} \theta) \\
\end{array}$ & & & $\langle h \overline{2 h}$ & & & & $(h \overline{2}$ & & & & & $2 \hbar \theta$ & & & \\
\hline$\eta_{60}$ & $\eta_{4 \mathrm{~A}}$ & \begin{tabular}{l|l}
$\eta_{48}$ & $\eta$
\end{tabular} & $\eta_{4 c}$ & $\eta_{40}$ & & $\eta_{4 E}$ & $\eta_{45}$ & $\eta_{46}$ & $\eta_{44}$ & & $\eta_{4 I}$ & $\eta_{40}$ & $\eta_{4 k}$ & $\eta_{4}$ & \\
\hline$h \overline{2 h} \theta)$ & & & & $(h \overline{2 h}$ & & & & & & $\bar{h}$ ब) & & & & $(h \overline{21}$ & h ब) \\
\hline$\eta_{6 E}$ & $\eta_{4 \mathrm{~A}}$ & $\eta_{46}$ & $4 B$ & $74 c$ & $n_{40}$ & & $4 E$ & $7_{4 \mathrm{~F}}$ & $\eta_{46}$ & $\eta_{44}$ & \begin{tabular}{|l|l|}
$7_{4 I}$ \\
\end{tabular} & $\eta_{4 \mathrm{~J}}$ & $\eta$ & $14 \mathrm{~K}$ & $74 \mathrm{~L}$ \\
\hline$h 2 h \theta)$ & & $\langle h \overline{2 h}$ & $\bar{h}$ ब) & & & & $2 \hbar$ ब) & & & & & & $\langle h \overline{2}$ & $2 \hbar$ ब) & \\
\hline$\eta_{6 \mathrm{~F}}$ & $\eta_{4 A}$ & $\eta_{4 B}$ & $\eta_{4 c}$ & & $\eta_{40}$ & \begin{tabular}{|l|l|}
$74 E$ \\
\end{tabular} & $\eta_{4}$ & & $\eta_{46}$ & $\eta_{4 \mathrm{H}}$ & & $74 I$ & & \begin{tabular}{l|l}
$j$ & $\eta_{4 k}$ \\
\end{tabular} & $\eta_{4 L}$ \\
\hline$h \overline{2 h} \theta)$ & & & $\langle h \overline{2 h}$ & & & & $\langle 4 \overline{2}$ & $\bar{h}$ ब) & & & & $2 \overline{25} 0$ & & & \\
\hline$\eta_{6 G}$ & & $\eta_{4 A}$ & $\eta_{48}$ & $\eta_{4 c}$ & $\eta_{40}$ & $\eta_{4 E}$ & $\eta_{4 \mathrm{~F}}$ & $\eta_{4}$ & & $\eta_{4 \mathrm{H}}$ & $77_{4 I}$ & & $74 \mathrm{~J}$ & $\eta_{4 k}$ & $\eta_{4 L}$ \\
\hline h2h & $\langle h \overline{2}$ & $\overline{2 h} \theta)$ & & & & & & $\langle h \overline{2}$ & $2 \hbar$ ब) & & & $\langle h \overline{2}$ & $\overline{2 h \theta)}$ & & \\
\hline$\eta_{66 \mathrm{H}}$ & $\eta_{4 \mathrm{~A}}$ & \begin{tabular}{l|l}
$\eta_{48}$ \\
\end{tabular} & $\eta_{4 c}$ & $\eta_{40}$ & & $\eta_{4 E}$ & $\eta_{4 F}$ & $\bar{n}_{46}$ & $\eta_{4}$ & & $\overline{\eta_{4 I}}$ & $\eta_{4 \mathrm{~J}}$ & $\eta_{4 k}$ & $\eta_{4}$ & \\
\hline$(h \overline{2 h} \theta)$ & & & \begin{tabular}{l|l} 
\\
\end{tabular} & $(h \overline{2 h}$ & & & & & (h) & (50) & & & & $\begin{array}{ll}h \overline{2} \\
\end{array}$ & $\bar{h}$ ब) \\
\hline$\eta_{6 I}$ & $74 \mathrm{~A}$ & $\eta_{48}$ & $\eta_{4 \mathrm{C}}$ & & $n_{40}$ & $7_{4 E}$ & $\eta_{4}$ & & $\eta_{46}$ & $\eta_{4 \mathrm{H}}$ & & 74 & $77_{4 \mathrm{~J}}$ & $\begin{array}{l} \\
\end{array}$ & $\eta_{4 L}$ \\
\hline$(h \overline{2 h} \theta)$ & & & $(h \overline{2 h}$ & & & & $\begin{array}{ll}l h \overline{2} \\
\end{array}$ & 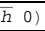 & & & $\langle(h \overline{2}$ & $2 h 0$ & & & \\
\hline $77_{6} 3$ & & $n_{4 A}$ & $\eta_{4 B}$ & $\eta_{4 c}$ & $\mid \eta_{40}$ & \begin{tabular}{|l|l|}
$\eta_{44}$ & \\
\end{tabular} & $7_{4 F}$ & $n_{4}$ & & $\eta_{44 \mathrm{H}}$ & $\eta_{4 I}$ & & $74 \mathrm{~J}$ & & $\eta_{4 L}$ \\
\hline$(h \overline{2 h} \quad \theta)$ & $\langle h \overline{2}$ & $\overline{2 h}$ ब) & & & & & & $\langle h \overline{2}$ & $2 \bar{h} \theta)$ & & & $(h \overline{2}$ & $2 \overline{2 h} \theta$ & & \\
\hline$\eta_{66 \mathrm{~K}}$ & $74 \mathrm{~A}$ & $\eta_{4}$ & $4 B$ & $77_{4 c}$ & 1740 & $\overline{\eta_{4}}$ & $4 E$ & $\eta_{4 F}$ & \begin{tabular}{l|l|}
$F$ & $\eta_{46}$ \\
\end{tabular} & $\eta_{4 H}$ & $7_{4 I}$ & $77 \mathrm{~J}$ & & $74 K$ & $\mid \eta_{4 L}$ \\
\hline$(h \overline{2 h} \theta)$ & & $(h \overline{21}$ & (h ब) & & & $(h \overline{2}$ & 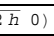 & & & & & & $\langle h \overline{2}$ & $2 \hbar$ O) & \\
\hline$\eta_{6 L}$ & $74 \mathrm{~A}$ & \begin{tabular}{l|l}
$\eta_{4 B}$ & 7
\end{tabular} & $\eta_{44}$ & $7 n_{40}$ & & $\eta_{4 E}$ & $\eta_{4 F}$ & $\eta_{46}$ & $\eta_{4}$ & & $\eta_{4} I$ & $77 \mathrm{~J}$ & | 746 & $\eta_{4}$ & \\
\hline$(h \overline{2 h} \theta)$ & & & & $(h \overline{2 h}$ & & & & & $\langle h \overline{21}$ & (h 0) & & & & $\langle h \overline{2 !}$ & $\overline{\bar{h}}$ 0) \\
\hline$\eta_{6 \in M}$ & & $74 A$ & $\eta_{4 B}$ & $\eta_{4 c}$ & $\mid 740$ & $\eta_{4 E}$ & $74 F$ & $\eta_{4}$ & 146 & $74 \mathrm{H}$ & $74 I$ & & & $74 k$ & $\eta_{4 L}$ \\
\hline$(h \overline{2 h} \theta)$ & $(\bar{h} 2$ & $2 h \theta)$ & & & & & & (द) 2 & $2 h \theta)$ & & & $\langle\bar{h} 2$ & $2 h \theta$ & & \\
\hline$\eta_{66 \mathrm{~N}}$ & \begin{tabular}{|l|}
$\eta_{4 A}$ \\
\end{tabular} & $\eta_{4}$ & & $\eta_{4 c}$ & $\eta_{40}$ & $\eta_{4}$ & $4 E$ & $\eta_{4 F}$ & \begin{tabular}{l|l}
$F$ & $\eta_{46}$ \\
\end{tabular} & $\eta_{4 H}$ & $\eta_{4 I}$ & $\eta_{4 \mathrm{~J}}$ & & $74 K$ & \\
\hline$\overline{h \overline{2 h} \theta)}$ & & $(\bar{h} 21)$ & $h$ o) & & & (h) 2 & $2 h \theta)$ & & & & & & $\langle\bar{h} 2$ & $2 h \theta$ & \\
\hline$\eta_{60}$ & \begin{tabular}{rl|}
$77_{4} \mathrm{~A}$ \\
\end{tabular} & 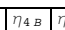 & $\mid \eta_{4 c}$ & $\eta_{40}$ & & $\eta_{4 E}$ & $\eta_{4 F}$ & $\eta_{46}$ & $\eta_{4}$ & & $\eta_{4 T}$ & $\eta_{4} \mathrm{~J}$ & $\eta_{4 k}$ & $\eta_{7_{4}}$ & \\
\hline$(h \overline{2 h} \theta)$ & & & & $(\bar{h} 2 h$ & & & & & $\left(\begin{array}{ll}\bar{h} & 2 ! \\
\end{array}\right.$ & $h$ ब) & & & & $\mid \begin{array}{ll}\bar{h} & 2 ! \\
\end{array}$ & $h$ ब) \\
\hline$\eta_{6 P}$ & $\eta_{4 A}$ & $\eta_{48}$ & $\eta_{44}$ & & $\eta_{40}$ & $7_{4 E}$ & $\eta_{4}$ & & $\eta_{4 G}$ & $\eta_{4 \mathrm{H}}$ & & $14 I$ & $7_{4 \mathrm{~J}}$ & \begin{tabular}{l|l}
$\eta_{4}$ & $\eta_{4 k}$
\end{tabular} & $\eta_{4 L}$ \\
\hline$(h \overline{2 h} \theta)$ & & & $(\bar{h} 2 h$ & h 0 ) & & & (h) 2 & $h$ ๑) & & & $\sqrt{\bar{h} 2}$ & $2 h$ ब) & & & \\
\hline$\eta_{60}$ & $\eta_{4 A}$ & $\eta_{4}$ & $4 B$ & \begin{tabular}{|l|}
$\eta_{4} c$ \\
\end{tabular} & $\eta_{40}$ & $\eta_{4}$ & $4 E$ & $\eta_{4 F}$ & \begin{tabular}{l|l}
$F$ & $\eta_{46}$
\end{tabular} & $\eta_{4 H}$ & $\eta_{4 I}$ & $\eta_{4 \mathrm{~J}}$ & $\eta$ & $74 K$ & $\eta_{4 L}$ \\
\hline$h \overline{2 h} \theta)$ & & $(\bar{h} 21$ & $h$ o) & & & $\begin{array}{ll}(\bar{h} 2 \\
\end{array}$ & $2 h 0)$ & & & & & & $(\bar{h} 2$ & $2 h \theta)$ & \\
\hline$\eta_{6 R}$ & \begin{tabular}{|l|l|}
$\eta_{4 A}$ \\
\end{tabular} & \begin{tabular}{l|l}
$\eta_{4 B}$ & $\eta$
\end{tabular} & $\eta_{4 c}$ & $\eta_{40}$ & & $\eta_{4 E}$ & \begin{tabular}{|l|}
$\eta_{4 F}$ \\
\end{tabular} & $\eta_{46}$ & & & $\eta_{4 I}$ & $\eta_{4} \mathrm{~J}$ & $\eta_{4 k}$ & $\eta_{4}$ & \\
\hline$n 2 h \theta)$ & & & \begin{tabular}{|l|l} 
& 1 \\
\end{tabular} & $(\bar{h} 2 h$ & & & & & 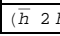 & $(n \theta)$ & & & & \begin{tabular}{|ll} 
(h & 21
\end{tabular} & $h \theta$ \\
\hline$\eta_{6 \mathrm{~s}}$ & & $\eta_{4 A}$ & $\eta_{4 B}$ & $\eta_{4 c} \mid$ & $\eta_{40}$ & $\eta_{4 E}$ & $\eta_{4 F}$ & & 746 & $\eta_{4 H}$ & $\eta_{41}$ & & $n_{43}$ & $\begin{array}{l}\eta_{4 k} \\
\end{array}$ & $\eta_{4 L}$ \\
\hline$(h \overline{2 h} \theta)$ & $\langle\bar{h} 2$ & & 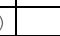 & & & & & (द⿳亠口冋 2 & $2 h \theta)$ & & & & $2 h \theta)$ & & \\
\hline$\eta_{6} \mathrm{~T}$ & $\eta_{4 \mathrm{~A}}$ & $\eta_{48}$ & $\eta_{4}$ & & $\eta_{40}$ & $\eta_{4 E}$ & $\eta_{4}$ & & $77_{46}$ & $\eta_{44}$ & & $74 I$ & $77_{43}$ & $\begin{array}{ll} & 74 k \\
\end{array}$ & $n_{4 L}$ \\
\hline$h \overline{2 h} \theta)$ & & & \begin{tabular}{|ll|l}
\multicolumn{1}{|c}{} & $2 !$
\end{tabular} & h0) & & & \begin{tabular}{ll}
\multicolumn{1}{l}{2} & 2 \\
\end{tabular} & 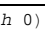 & & & & $2 h \theta$ & & & \\
\hline$\eta_{66}$ & $\eta_{4 A}$ & $\eta_{48} T_{7}$ & \begin{tabular}{|l|l|}
$\eta_{4 c}$ & \\
\end{tabular} & $\eta_{40}$ & & \begin{tabular}{|l|l|}
$\eta_{4 E}$ & \\
\end{tabular} & $\eta_{4 F}$ & $\eta_{46}$ & $\eta_{4}$ & & $\eta_{41}$ & $\eta_{4} \mathrm{~J}$ & $\eta_{4 k}$ & $\eta_{4}$ & \\
\hline$h 2 h \theta$ & & & & $(\bar{h} 2 h$ & & & & & (h) 2 & $2 h \theta)$ & & & & $\begin{array}{lll}\text { (h } & 21\end{array}$ & $h$ o) \\
\hline$\eta_{6 \mathrm{~V}}$ & & $\eta_{4 A}$ & $\eta_{4 B}$ & $\eta_{4 c}$ & $n_{40}$ & $\eta_{4 E}$ & $\eta_{4 F}$ & & 746 & $\eta_{4 H}$ & $\eta_{4 I}$ & & $74 \mathrm{~J}$ & $\eta_{4 k}$ & $\eta_{4 L}$ \\
\hline$(h 2 h \theta)$ & (h 2 & $2 h \theta$ & & & & & & (h 2 & $2 h \theta)$ & & & $\left(h^{2}\right.$ & $2 h \theta)$ & & \\
\hline$n_{6 \mathrm{w}}$ & $74 \mathrm{~A}$ & $\eta_{4}$ & & $\eta_{4 c}$ & $\eta_{40}$ & $\eta_{4}$ & $4 E$ & $\eta_{4 F}$ & \begin{tabular}{l|l|l|}
$F$ & $\eta_{46}$
\end{tabular} & \begin{tabular}{l|l}
6 & $\eta_{4 H}$
\end{tabular} & $\eta_{4 I}$ & $\eta_{4 \mathrm{~J}}$ & $n$ & $4 k$ & $\eta_{4 L}$ \\
\hline$(h \overline{2 h} \theta)$ & & \begin{tabular}{lll}
\multicolumn{1}{l}{} & 21
\end{tabular} & $2 h \theta$ & & & \begin{tabular}{|l|}
\multicolumn{1}{l}{. } \\
\end{tabular} & $2 h$ Q) & & & & & & & $2 h \theta$ & \\
\hline $76 x$ & $74 \mathrm{~A}$ & $\eta_{48}$ & $\eta_{4}$ & & $\eta_{40}$ & \begin{tabular}{|l|l|}
${ }^{2}$ & \\
\end{tabular} & $\eta_{4}$ & & $77_{46}$ & $\eta_{4 H}$ & & $74 I$ & $\begin{array}{l}\eta_{4.5} \\
\end{array}$ & $\begin{array}{l} \\
\end{array}$ & $\eta_{4 L}$ \\
\hline$(h \overline{2 h} \theta)$ & & & $\mid \begin{array}{ll}\bar{h} & 21 \\
\end{array}$ & $\begin{array}{ll}h \text { o) } \\
\end{array}$ & & & $\langle\sqrt{\hbar \bar{h} 2}$ & $h$ ब) & & & & $2 h \theta$ & & & \\
\hline
\end{tabular}


$\left(\eta_{6}, \eta_{5}\right)$

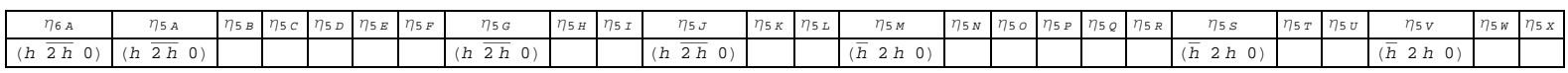

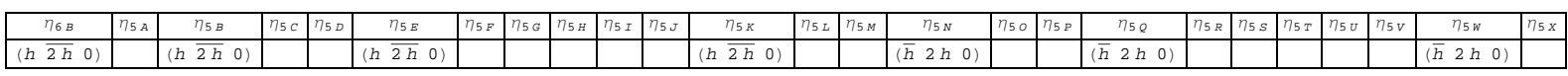

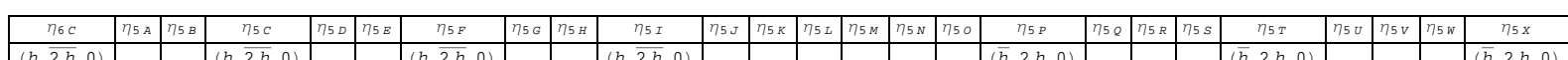

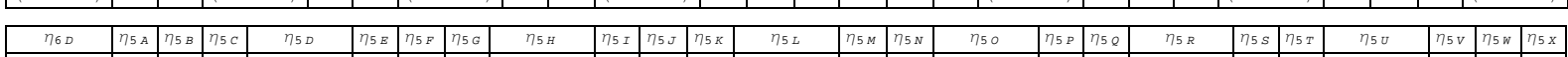

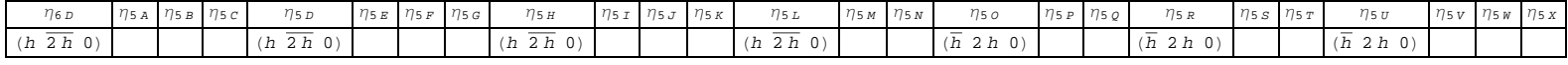

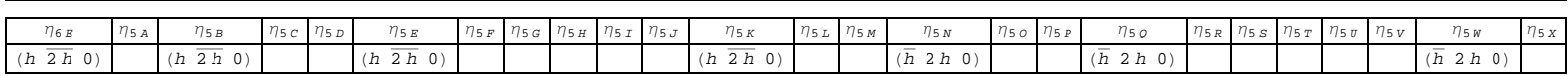

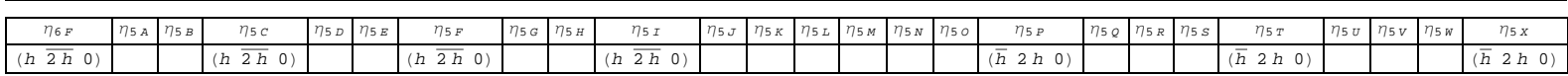

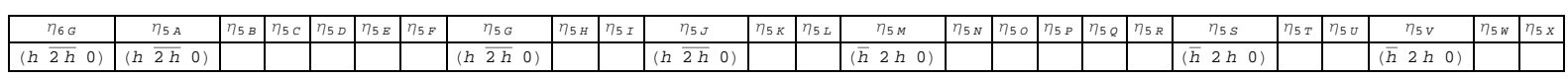

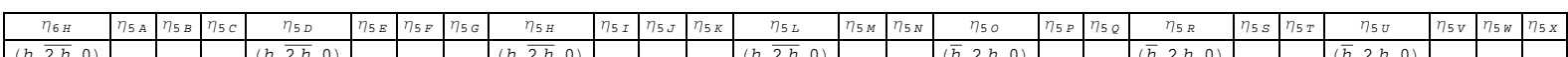

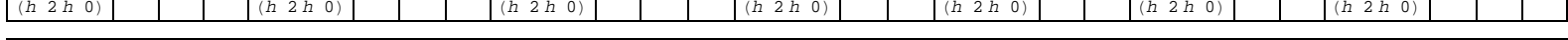

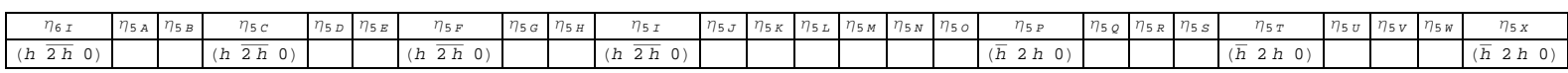

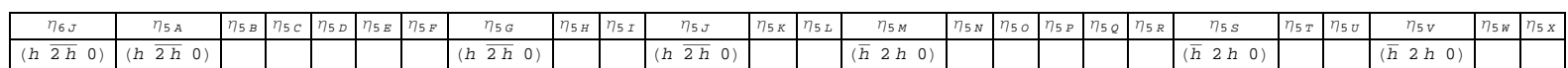

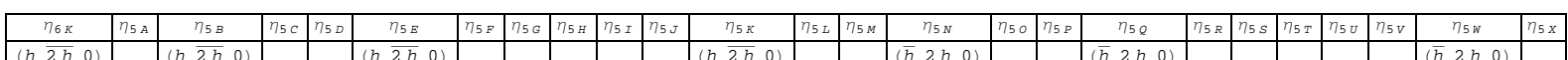

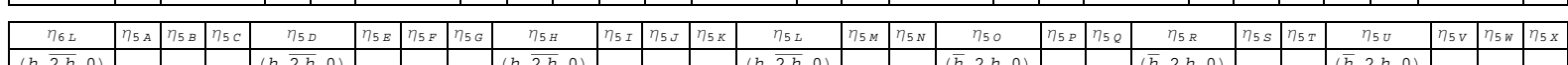

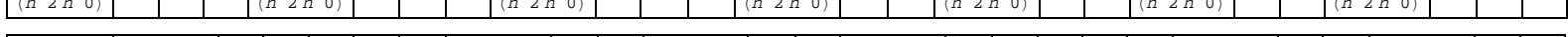

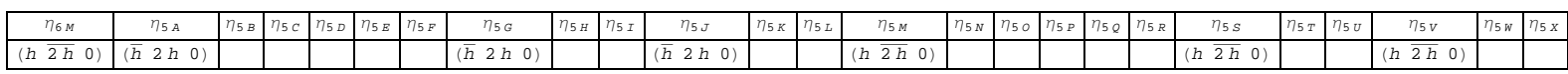

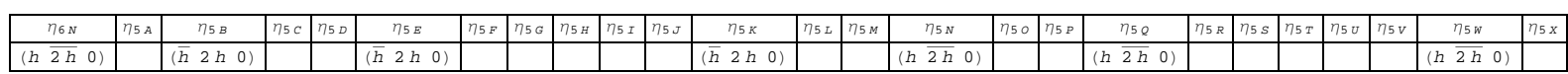

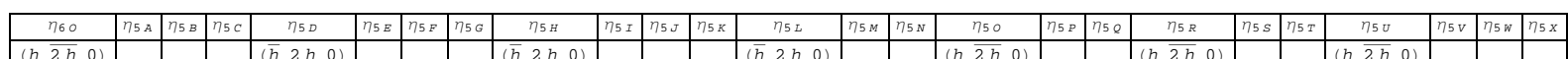

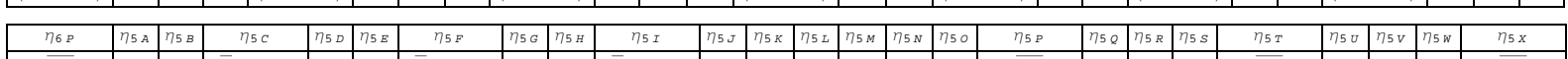

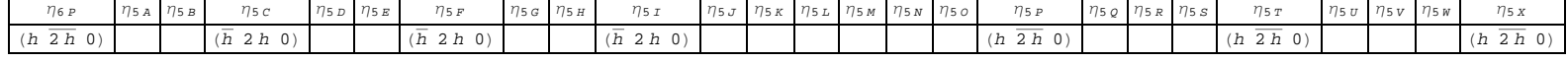

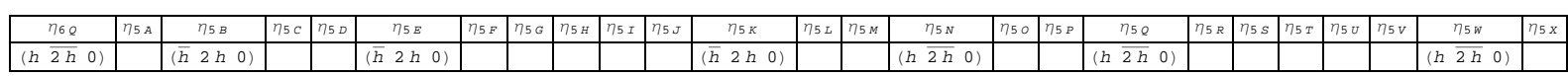

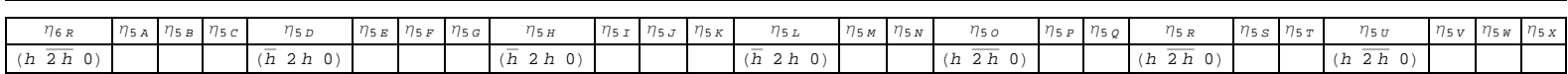

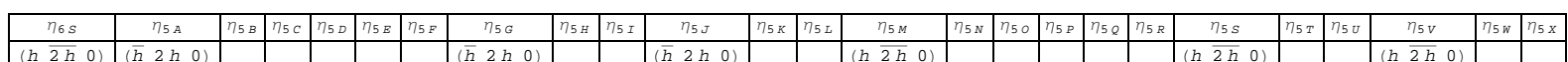

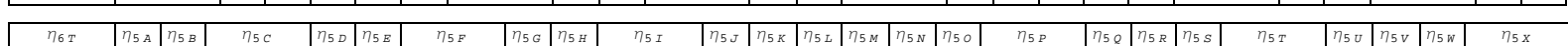

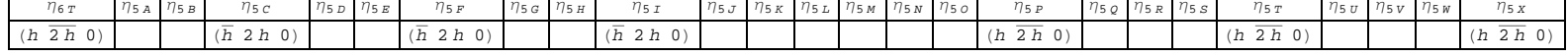

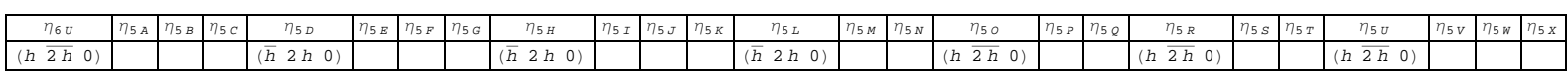

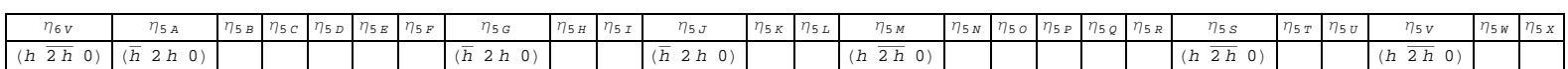

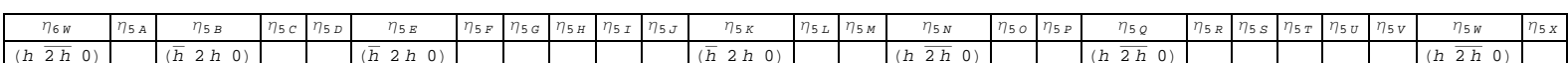

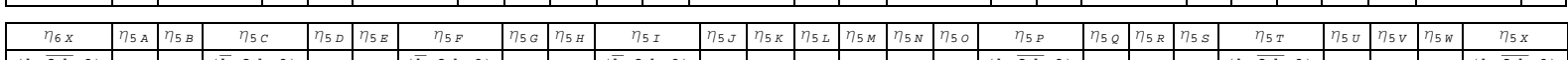

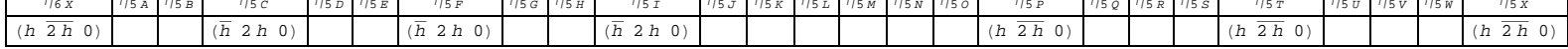


$\left(\eta_{6}, \eta_{7}\right)$

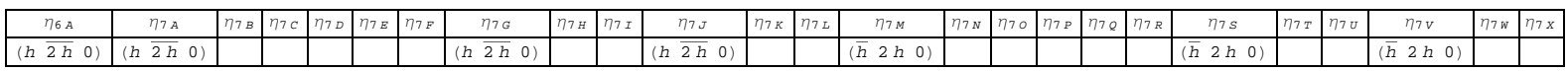

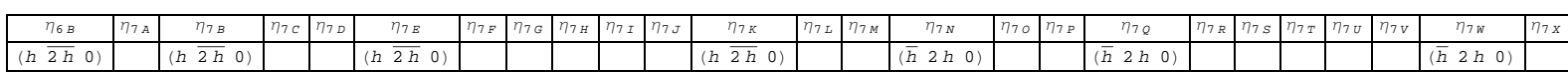

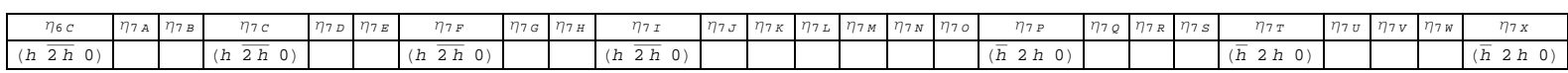

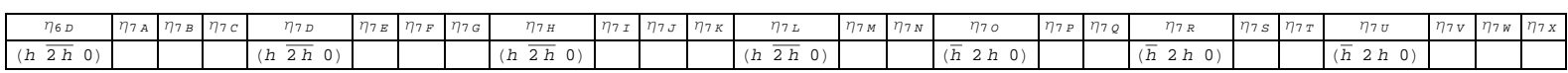

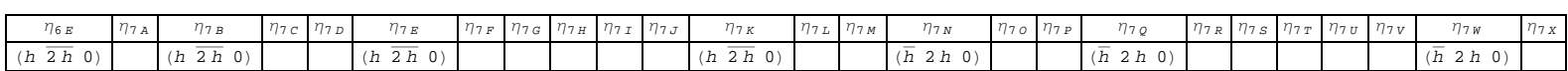

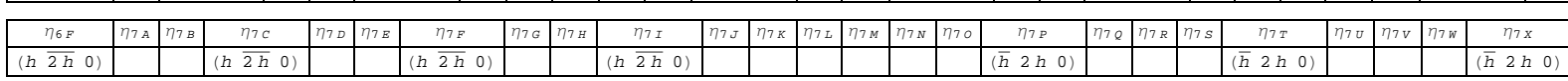
\begin{tabular}{|c|c|c|c|c|c|c|c|c|c|c|c|c|c|c|c|c|c|c|c|c|c|c|c|c|}
\hline$\eta_{66}$ & $\eta_{7 \mathrm{~A}}$ & $\eta_{78}$ & $\eta_{7 \mathrm{C}}$ & $\eta_{70}$ & $\eta_{7 \mathrm{E}}$ & $\eta_{7 \mathrm{~F}}$ & $\eta_{76}$ & $\eta_{7 \mathrm{H}}$ & $\eta_{7 \mathrm{I}}$ & $\eta_{7 J}$ & $\eta_{7 \mathrm{~K}}$ & $\eta_{7 \mathrm{~L}}$ & $\eta_{7 \mathrm{M}}$ & $\eta_{7 \mathrm{~N}}$ & $\eta_{70}$ & $\eta_{7 \mathrm{P}}$ & $\eta_{7 \mathrm{Q}}$ & $\eta_{7 \mathrm{R}}$ & $\eta_{7 \mathrm{~S}}$ & $\eta_{7 \mathrm{~T}}$ & $\eta_{7 \mathrm{U}}$ & $\eta_{7 \mathrm{~V}}$ & $\eta_{7 \mathrm{~W}}$ & $\eta_{7 \mathrm{X}}$ \\
\hline
\end{tabular}

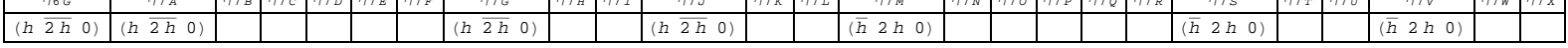

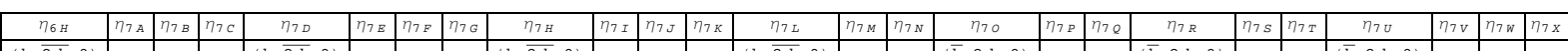

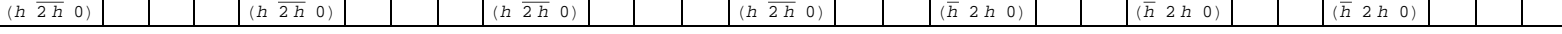

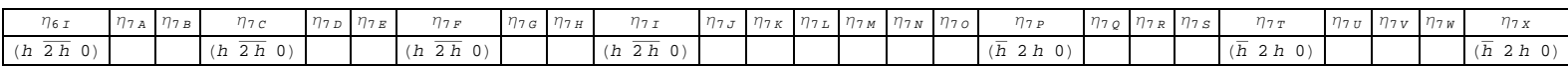

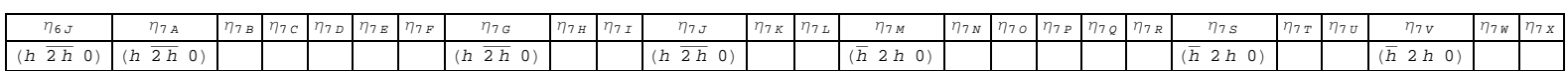

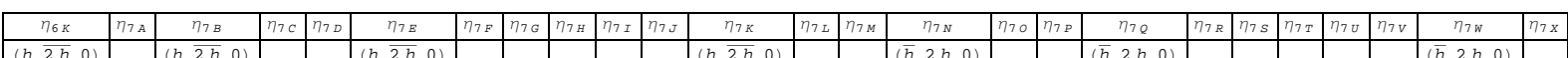

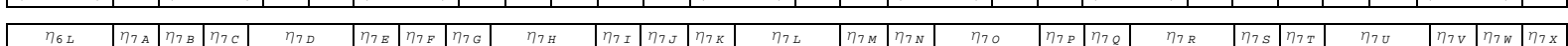

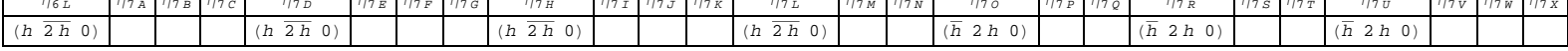

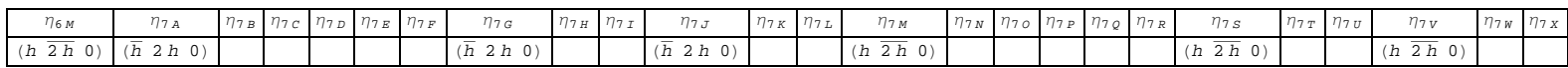

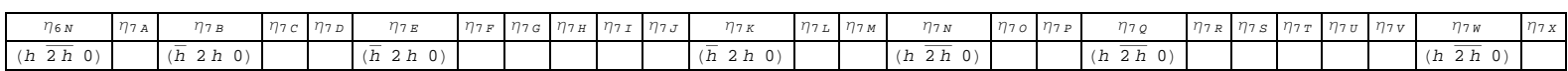

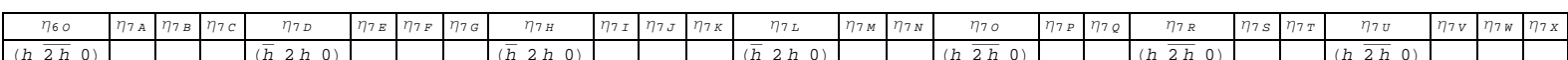

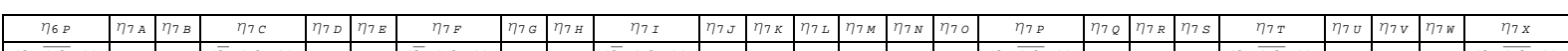
\begin{tabular}{|l|l|l|l|l|l|l|l|l|l|l|l|l|l|l|l|}
\hline$(h \overline{2 h} \theta)$ & & $(\bar{h} 2 h \theta)$ & $(\bar{h} 2 h \theta)$ & & $(\bar{h} 2 h \theta)$ & & & & & $(h \overline{2 h} \theta)$ & & & $(h \overline{2} \theta)$ & & $(h \overline{2 h} \theta)$ \\
\hline
\end{tabular}

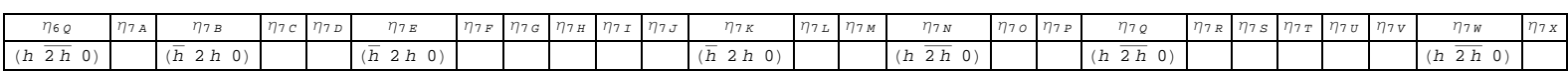

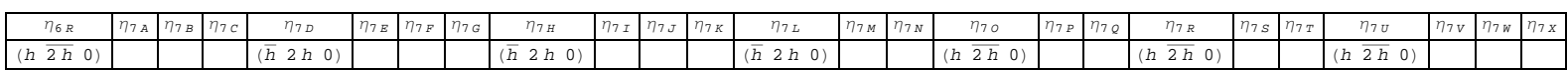

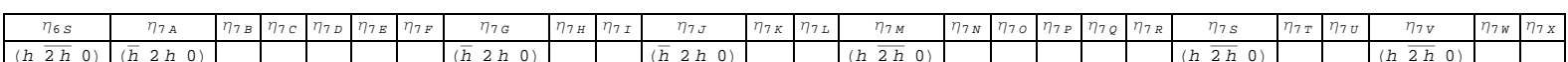

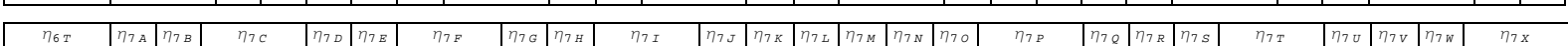

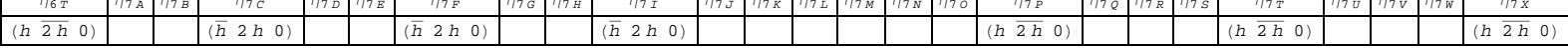

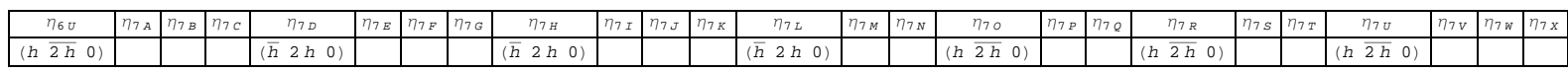

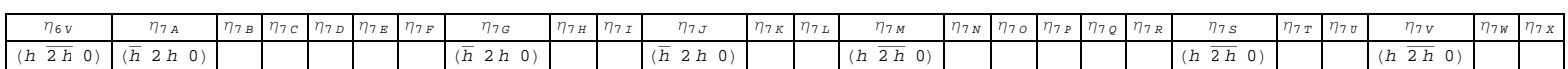

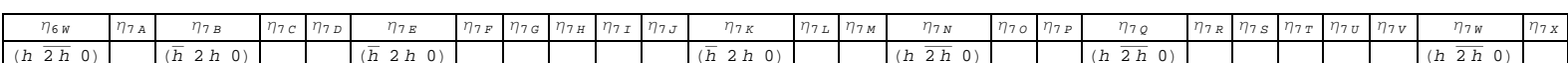

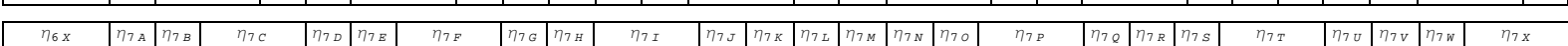

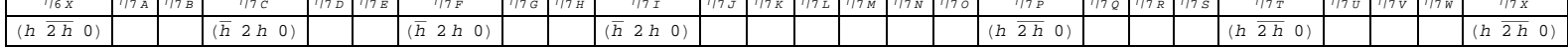


$\left(\eta_{7}, \eta_{4}\right)$

\begin{tabular}{|c|c|c|c|c|c|c|c|c|c|c|c|c|c|c|}
\hline$\eta_{7 \mathrm{~A}}$ & \multirow{2}{*}{\multicolumn{2}{|c|}{$\frac{\eta_{4 A}}{(h \overline{2 h} \theta)}$}} & \begin{tabular}{|l|l|}
748 \\
\end{tabular} & \begin{tabular}{l|l}
$\eta_{44}$ & $n$ \\
\end{tabular} & $n_{40}$ & \begin{tabular}{ll|}
$7_{4 E}$ \\
\end{tabular} & $\eta_{45}$ & \multirow{2}{*}{\begin{tabular}{|c}
$\eta_{4 G}$ \\
$(h \overline{2 h} \Theta)$
\end{tabular}} & \multirow[t]{2}{*}{ (44H } & $\eta_{4 I}$ & \multirow{2}{*}{\multicolumn{2}{|c|}{\begin{tabular}{|c}
$\eta_{4 J}$ \\
$(h \overline{2 h} \theta)$
\end{tabular}}} & \multirow[t]{2}{*}{\begin{tabular}{|l|l|} 
& \\
\end{tabular}} & \multirow[t]{2}{*}{$\eta_{4 L}$} \\
\hline$(h \overline{2 h} \theta)$ & & & & & & & & & & & & & & \\
\hline$\eta_{7 B}$ & $\eta_{4 A}$ & & $74 \mathrm{~B}$ & \begin{tabular}{l|l}
$7_{44 c}$ & $\eta$ \\
\end{tabular} & $\eta_{40}$ & & $14 \mathrm{E}$ & $\begin{array}{ll}\eta_{45} & \eta_{46}\end{array}$ & $\eta_{4 H}$ & $\eta_{4 I}$ & $\eta_{4 \mathrm{~J}}$ & $\eta_{4}$ & & $\begin{array}{l}\eta_{4 L} \\
\end{array}$ \\
\hline$h \overline{2 h} \theta)$ & & & $2 h \quad 0)$ & & & & $2 h \quad 0)$ & & & & & & (50) & \\
\hline$\eta_{7 c}$ & $\mid \begin{array}{ll}\eta_{4 A} \\
\end{array}$ & $\mid \begin{array}{l}n_{48} \\
\end{array}$ & $\eta_{4 c}$ & & $\eta_{40}$ & $\eta_{4 E}$ & $\eta_{4}$ & $\mid \eta_{46}$ & $\eta_{4 \mathrm{H}}$ & & $7_{44}$ & $\eta_{4 J}$ & $\eta_{4 k}$ & $\eta_{4 L}$ \\
\hline$(h \overline{2 h} \theta)$ & & & $\langle h \overline{2 h}$ & & & & $\mid l h \overline{21}$ & (he) & & $\begin{array}{ll}(h \overline{2} \\
\end{array}$ & $2 \overline{2 h} 0)$ & & & \\
\hline$\eta_{70}$ & $74 \mathrm{~A}$ & $\eta_{48}$ & $\eta_{4 c}$ & $\eta_{40}$ & & $7_{4 E}$ & $\begin{array}{ll}77_{4 F} \\
\end{array}$ & \begin{tabular}{|l|l}
$\eta_{46}$ & $\eta_{4}$ \\
\end{tabular} & & $\eta_{4 I}$ & $7_{44}$ & $7_{4 K}$ & $\eta_{4}$ & \\
\hline$h \overline{2 h} \theta)$ & & & & $(h \overline{2 h}$ & (5) & & & \begin{tabular}{|l|ll} 
& $(h \overline{2}$
\end{tabular} & (h ब) & & & & & 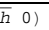 \\
\hline$\eta_{7 E}$ & $74 \mathrm{~A}$ & 7 & 748 & \begin{tabular}{|l|l|}
$\eta_{40}$ & $\eta$ \\
\end{tabular} & & & $74 \mathrm{E}$ & \begin{tabular}{|l|l|}
$\eta_{44}$ & $\eta_{4 G}$ \\
\end{tabular} & \begin{tabular}{l|l}
6 & $\eta_{44 \mathrm{H}}$
\end{tabular} & $\eta_{4 I}$ & $\eta_{43}$ & $\eta_{4}$ & & $\eta_{4 L}$ \\
\hline$(h \overline{2 h} \theta)$ & & 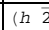 & $2 \overline{2 h} \theta)$ & & & $\langle h \overline{2}$ & $2 \mathrm{~h} \theta)$ & & & & & $\langle h \overline{2}$ & h ब) & \\
\hline$\eta_{7 \mathrm{~F}}$ & $\eta_{4 A}$ & $\eta_{48}$ & $\eta_{4 c}$ & & $\eta_{40}$ & $\eta_{4 E}$ & $\eta_{4}$ & $\eta_{4 G}$ & $\eta_{4 H}$ & & $74 I$ & $77_{4 \mathrm{~J}}$ & $\eta_{4 k}$ & $\eta_{4 L}$ \\
\hline $\begin{array}{ll}n 2 h \theta) \\
\end{array}$ & & & $\langle h \overline{2 h}$ & & & & $\mid \begin{array}{ll}h & \overline{2} \mid \\
\end{array}$ & (50) & & $\begin{array}{ll}h & \overline{2} \\
\end{array}$ & $2 \hbar \Theta$ & & & \\
\hline$\eta_{7 G}$ & & $r_{4 A}$ & $\eta_{48}$ & \begin{tabular}{|l|l|l}
$\eta_{4 c}$ & $\gamma$ \\
\end{tabular} & $\eta_{40}$ & $\eta_{4 E}$ & $\eta_{4 F}$ & $\eta_{4 G}$ & $\eta_{44 \mathrm{H}}$ & $\mid$ & & (14j & \begin{tabular}{|l|l|}
$n_{4 k}$ \\
\end{tabular} & $\eta_{4 L}$ \\
\hline$(h \overline{2 h} \theta)$ & $\langle h \overline{2}$ & $2 \overline{2 h \theta}$ & & & & & & $\langle(h \overline{2 h} \theta\rangle$ & & & $\langle h \overline{2}$ & $\overline{2 h}$ ब) & & \\
\hline$\eta_{7 \mathrm{H}}$ & $74 \mathrm{~A}$ & $\eta_{48}$ & $\eta_{4 c}$ & $\eta_{40}$ & & $74 E$ & \begin{tabular}{ll|l}
$7_{45}$ & 7 \\
\end{tabular} & \begin{tabular}{|l|l}
$\eta_{46}$ & $\eta_{4}$ \\
\end{tabular} & & $\eta_{4 I}$ & $\eta_{4 \mathrm{~J}}$ & $\eta_{4 k}$ & $\eta_{4}$ & \\
\hline$(h \overline{2 h} \theta)$ & & & & $(h \overline{2 h}$ & & & & \begin{tabular}{|l|l} 
& $(h \overline{2}$
\end{tabular} & & & & & $\langle h \overline{2 h}$ & $\bar{h}$ ब) \\
\hline$\eta_{7 I}$ & $\eta_{4 \mathrm{~A}}$ & $\eta_{48}$ & $\eta_{4 c}$ & & \begin{tabular}{|l|}
$7_{40}$ \\
\end{tabular} & $\eta_{4 E}$ & $\eta_{4}$ & $\eta_{4 G}$ & $\eta_{44}$ & & $74 I$ & $74 \mathrm{~J}$ & $74 \mathrm{~K}$ & $\eta_{4 L}$ \\
\hline$(h \overline{2 h} \theta)$ & & & $\langle h \overline{2 h}$ & $\begin{array}{l}\bar{h} 0) \\
\end{array}$ & & & $(h \overline{21}$ & $\left(\begin{array}{ll}n & 0)\end{array}\right.$ & & $\begin{array}{ll}h \overline{2} \\
\end{array}$ & $2 h \Theta$ & & & \\
\hline$\eta_{7 \mathrm{~J}}$ & & $74 \mathrm{~A}$ & $\eta_{4 B}$ & \begin{tabular}{|l|l}
$\eta_{4}$ & 17
\end{tabular} & $7_{40}$ & \begin{tabular}{|l|}
$\eta_{4 E}$ \\
\end{tabular} & $\eta_{4 F}$ & $\eta_{46}$ & $\eta_{44}$ & $\eta_{4 I}$ & & $74 \mathrm{~J}$ & $7_{4 k}$ & $\eta_{4 L}$ \\
\hline$(h \overline{2 h} \theta)$ & $\langle 4 \overline{2}$ & $\overline{2 h \theta}$ & & & & & & $\langle(h \overline{2 h} \theta\rangle$ & & & $\langle h \overline{2}$ & $2 \hbar$ ब & & \\
\hline$\eta_{77 K}$ & 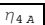 & & 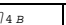 & \begin{tabular}{|l|l|l}
$\eta_{4 c}$ & \\
\end{tabular} & & & (4E & \begin{tabular}{|l|l|l}
$n_{4 F}$ & $n_{46}$
\end{tabular} & $\eta_{4 H}$ & $\mid$ & $\eta_{43}$ & $\eta_{4}$ & & $n_{4 L}$ \\
\hline$(h \overline{2 h} \theta)$ & & $\mid(h \overline{2}$ & $2 \mathrm{~h} \theta)$ & & & & $2 \overline{2 h} 0)$ & & & & & $\begin{array}{ll}h & \overline{2} \\
\end{array}$ & (ho) & \\
\hline$\eta_{7 L}$ & $74 \mathrm{~A}$ & $7_{48}$ & $77_{4 c}$ & $\eta_{40}$ & & \begin{tabular}{ll|}
$\eta_{4 E}$ \\
\end{tabular} & \begin{tabular}{|l|l}
$7_{44}$ & 7 \\
\end{tabular} & \begin{tabular}{|l|l}
$\eta_{46}$ & $\eta_{4}$ \\
\end{tabular} & & $74 I$ & $74 \mathrm{~J}$ & $77_{4 K}$ & $\eta_{44}$ & \\
\hline$(h \overline{2 h} \theta)$ & & & & $(h \overline{2 h}$ & & & & \begin{tabular}{|l|ll} 
& $(h \overline{2}$ \\
\end{tabular} & $\bar{h}$ ब) & & & & $\langle h \overline{2 h}$ & $\bar{h}$ ब) \\
\hline$\eta_{7>M}$ & & $74 A$ & \begin{tabular}{|l|}
$7 \eta_{48}$ \\
\end{tabular} & \begin{tabular}{l|l|l}
$\eta_{4}$ & 7
\end{tabular} & 740 & \begin{tabular}{|l|l|}
$\eta_{4}$ & \\
\end{tabular} & $74 \mathrm{~F}$ & $\eta_{4 G}$ & $74 \mathrm{H}$ & $7_{4 I}$ & $\eta_{4}$ & $74 \mathrm{~J}$ & $n_{4 k}$ & $\eta_{4 L}$ \\
\hline$(h \overline{2 h} \theta)$ & $\langle\bar{h} 2$ & $2 h 0$ & & & & & & $(\bar{h} 2 h \quad \theta)$ & & & (h) 2 & $2 h \quad 0)$ & & \\
\hline$n_{7 N}$ & $\eta_{4 A}$ & & $74 B$ & \begin{tabular}{|l|l|}
$\eta_{4 c}$ & $r$ \\
\end{tabular} & $n_{40}$ & & $74 \mathrm{E}$ & \begin{tabular}{|l|l|}
$\eta_{44}$ & $\eta_{46}$
\end{tabular} & $\eta_{4 H}$ & $\eta_{4 I}$ & $\mid \eta_{4 J}$ & $\eta_{4}$ & & $\mid \eta_{4 L}$ \\
\hline$(h \overline{2 h} \theta)$ & & $\langle\bar{h} 2$ & $2 h \theta)$ & & & $(\bar{h} 2$ & $2 h \theta$ & & & & & \begin{tabular}{ll}
\multicolumn{1}{|l}{2} \\
\end{tabular} & $h$ o) & \\
\hline$\eta_{70}$ & $74 \mathrm{~A}$ & $7_{4 B}$ & $\eta_{4 c}$ & $\eta_{40}$ & & $\eta_{4 \mathrm{E}}$ & \begin{tabular}{|l|l}
$\eta_{44}$ & 7 \\
\end{tabular} & \begin{tabular}{|l|l}
$\eta_{46}$ & $\eta_{4}$ \\
\end{tabular} & & $\eta_{4 I}$ & $77_{4} \mathrm{~J}$ & \begin{tabular}{|l|l|}
$74 K$ \\
\end{tabular} & $\eta_{44}$ & \\
\hline$(h \overline{2 h} \theta)$ & & & & $(\bar{h} 2 h$ & & & & \begin{tabular}{|l|ll} 
& $(\bar{h}$ & 2 \\
\end{tabular} & $h$ e) & & & & $(\bar{h} 2 h$ & $h \theta$ \\
\hline$\eta_{7 P}$ & $\eta_{4 A}$ & $7_{48}$ & $\eta_{4 c}$ & & 740 & $\eta_{4 E}$ & $\eta_{4}$ & $\eta_{46}$ & $\eta_{4 \mathrm{H}}$ & & $74 I$ & $74 \mathrm{~J}$ & $\eta_{4 k}$ & $\eta_{4 L}$ \\
\hline$(h \overline{2 h} \theta)$ & & & $\langle\bar{h} 2 h$ & $h \theta$ & & & $\left\langle\begin{array}{ll}\bar{h} & 21 \\
\end{array}\right.$ & $h \quad 0)$ & & & $2 h \theta$ & & & \\
\hline$\eta_{7 Q}$ & $\eta_{4 A}$ & $n$ & & \begin{tabular}{|l|l}
$\eta_{4 c}$ & $r$ \\
\end{tabular} & $\eta_{40}$ & & & \begin{tabular}{|l|l}
$\eta_{4 F}$ & $\eta_{4 G}$
\end{tabular} & $\eta_{4 H}$ & $\eta_{4 I}$ & $\eta_{40}$ & $\eta_{4}$ & & $\eta_{4 L}$ \\
\hline$h \overline{2 h} \theta)$ & & $4 \overline{\hbar n} 2$ & $2 h \theta)$ & & & $\mid \bar{\hbar} 2$ & $2 h \theta$ ) & & & & & $\langle\bar{h} 2$ & $h \theta$ () & \\
\hline$\eta_{7 R}$ & $\eta_{4 A}$ & $\eta_{4 B}$ & \begin{tabular}{|l|l}
$\eta_{4 c}$ \\
\end{tabular} & $\eta_{40}$ & & $\eta_{4 E}$ & & $\eta_{46}$ & & $\eta_{41}$ & $\eta_{43}$ & $7_{4 K}$ & $\eta_{4}$ & \\
\hline$(h 2 h \theta)$ & & & $\begin{array}{ll}18 \\
\end{array}$ & $\left(\begin{array}{ll}h 2 h \\
\end{array}\right.$ & & & & \begin{tabular}{|l|ll|} 
& \multicolumn{1}{l}{2} \\
\end{tabular} & $2 h \theta)$ & & & & $\begin{array}{ll}4 & 2 h \\
\end{array}$ & $\begin{array}{ll}h \theta) \\
\end{array}$ \\
\hline$\eta_{7 s}$ & & $n_{4 A}$ & $\eta_{48}$ & $7_{4 c} \mathrm{r}$ & 740 & \begin{tabular}{|l|}
$7_{4 E}$ \\
\end{tabular} & \begin{tabular}{|l|}
$74 F$ \\
\end{tabular} & \begin{tabular}{|l|l}
$\eta_{46}$ \\
\end{tabular} & $74 \mathrm{H}$ & $\eta_{4 I}$ & & 743 & $7_{4 k}$ & $\eta_{4 L}$ \\
\hline$(h \overline{2 h} \theta)$ & & $2 h \theta$ & & & & & & 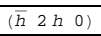 & & & & $2 h \quad \theta)$ & & \\
\hline$\eta_{7 T}$ & $\eta_{4 A}$ & $\eta_{48}$ & $\eta_{4 c}$ & & $7_{40}$ & $\eta_{4 E}$ & $\eta_{4}$ & \begin{tabular}{l|l}
$\mathrm{HF}$ & $\eta_{46}$
\end{tabular} & $\eta_{4 \mathrm{H}}$ & $\eta_{4}$ & $74 I$ & $\begin{array}{l}7_{43} \\
\end{array}$ & $7_{4 k}$ & $\eta_{4 L}$ \\
\hline$(h \overline{2 h} \theta)$ & & & 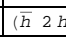 & 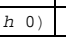 & & & \begin{tabular}{|lll}
\multicolumn{1}{l}{} & 21
\end{tabular} & $\begin{array}{ll}h & 0 \\
\end{array}$ & & & $2 h \theta$ & & & \\
\hline$\eta_{70}$ & $\eta_{4 \mathrm{~A}}$ & $\eta_{48}$ & $\eta_{4 c}$ & $\eta_{4 D}$ & & $\eta_{4 E}$ & \begin{tabular}{|l|l}
$\eta_{4 F}$ & 7 \\
\end{tabular} & \begin{tabular}{|l|}
746 \\
7
\end{tabular} & & $\mid$\begin{tabular}{l|}
$\eta_{4 I}$ \\
\end{tabular} & $\eta_{4} \mathrm{~J}$ & $\eta_{4 k}$ & $\eta_{4}$ & 44 \\
\hline$(h 2 h \theta)$ & & & 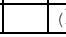 & $\left(\begin{array}{ll}h & 2 h \\
\end{array}\right.$ & & & & \begin{tabular}{|l|ll} 
& (h 2 \\
\end{tabular} & $2 n \theta$ () & & & & \begin{tabular}{ll}
\multicolumn{1}{l}{$22 h$} \\
\end{tabular} & $h$ Q) \\
\hline$\eta_{7 \mathrm{v}}$ & & $74 A$ & $\eta_{4 B}$ & $\eta_{4 c}$ & $\eta_{40}$ & \begin{tabular}{|l|l|}
$7_{4}$ & \\
\end{tabular} & \begin{tabular}{|l|l|}
$\eta_{4}$ & \\
\end{tabular} & $\eta_{4 G}$ & $\eta_{44 \mathrm{H}}$ & $\eta_{4 I}$ & $\eta_{4}$ & $74 \mathrm{~J}$ & $\eta_{4 k}$ & $\eta_{4 L}$ \\
\hline$(h 2 h \theta)$ & & $2 h \theta)$ & & & & & & \begin{tabular}{|llll}
$h$ & $2 h$ & $\theta$
\end{tabular} & & & & $2 h \quad \theta$ & & \\
\hline$n_{7 w}$ & $74 \mathrm{~A}$ & $\eta$ & 748 & $\eta_{4 c}$ & $n_{40}$ & & $74 E$ & \begin{tabular}{|l|l|}
$\eta_{4} \mathrm{~F}$ & $\eta_{46}$ \\
\end{tabular} & $\eta_{4 H}$ & $7_{4 I}$ & $\eta_{4 \mathrm{~J}}$ & $\eta_{4}$ & & $\eta_{4 L}$ \\
\hline$(h \overline{2 h} \theta)$ & & $1 \overline{152}$ & $2 h \theta)$ & & & $\langle$ (h 2 & $2 h \theta)$ & & & & & \begin{tabular}{ll}
\multicolumn{1}{l}{$h_{2}$} \\
\end{tabular} & $2 h 0)$ & \\
\hline$n_{77 x}$ & $\eta_{4 \mathrm{~A}}$ & $7_{48}$ & $\eta_{44}$ & $4 c$ & $7_{40}$ & \begin{tabular}{|l|l|}
$\eta_{4 E}$ \\
\end{tabular} & $\eta_{4}$ & \begin{tabular}{l|l}
$4 F$ & $\eta_{46}$
\end{tabular} & $\eta_{44}$ & & $74 I$ & $\eta_{43}$ & $74 \mathrm{~K}$ & $\eta_{4 L}$ \\
\hline$(h \overline{2 h} \theta)$ & & & $\mid \bar{h} 2 h$ & $h$ ब) & & & 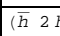 & \begin{tabular}{|c|c|}
$h$ & 0 \\
\end{tabular} & & $\langle\bar{h} 2$ & $2 h \theta$ & & & \\
\hline
\end{tabular}


$\left(\eta_{7}, \eta_{5}\right)$

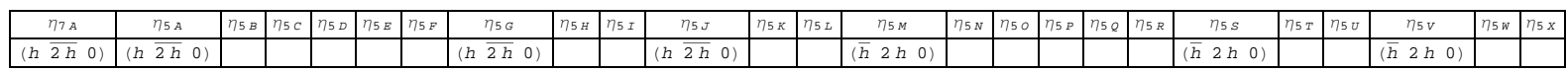

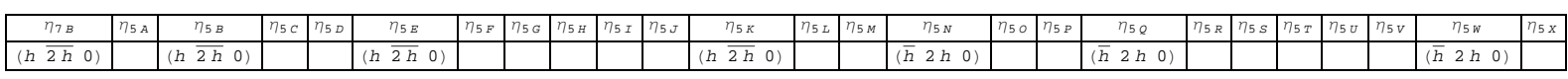

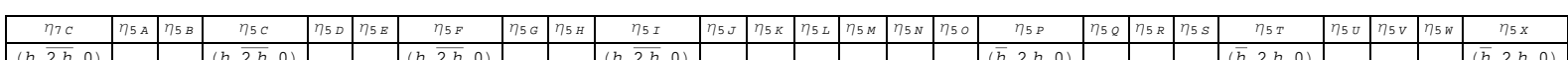

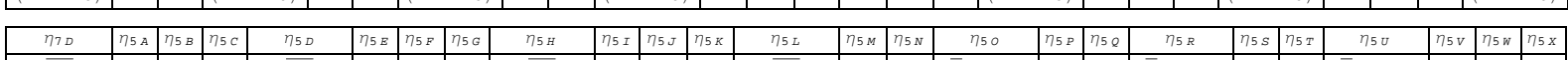

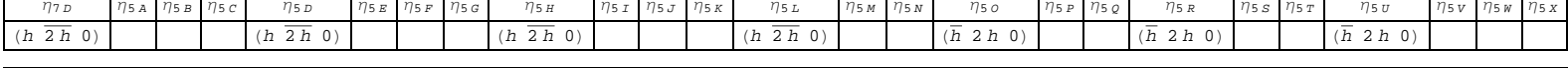

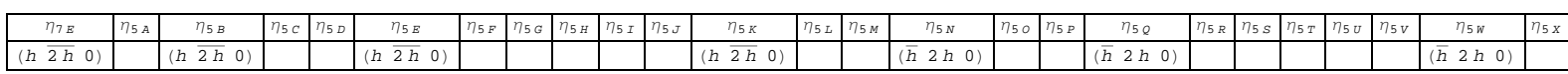

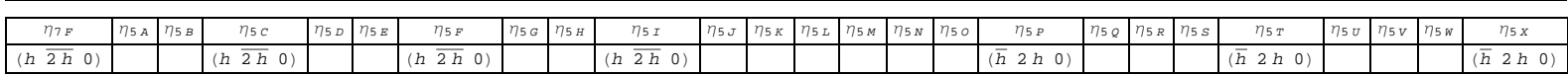

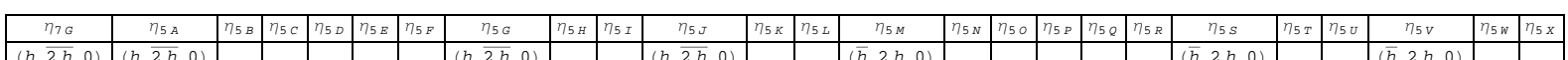

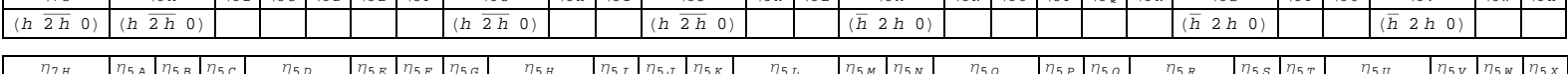

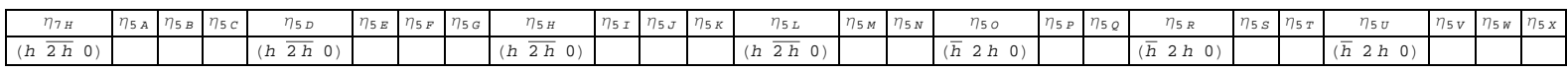

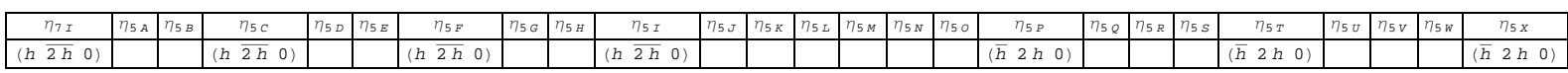

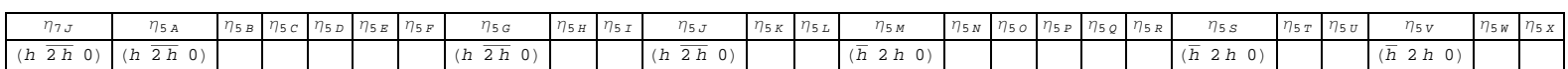

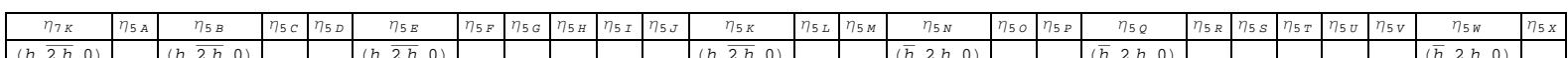

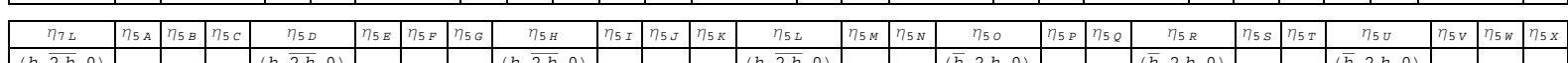

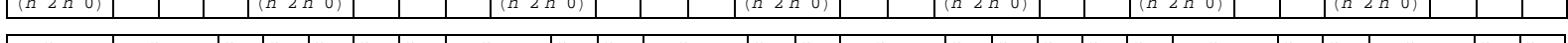

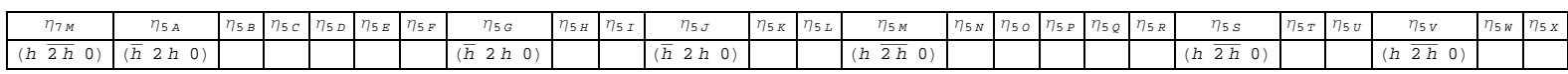

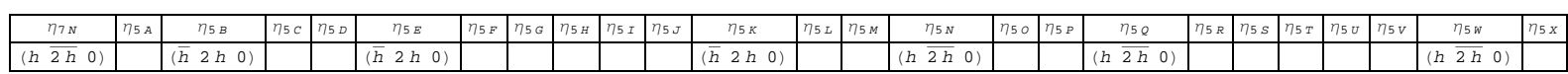

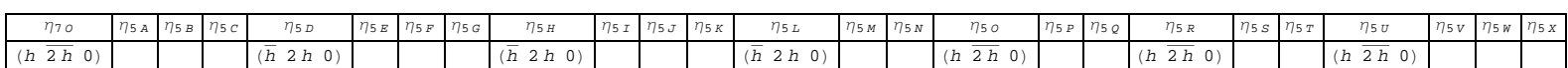

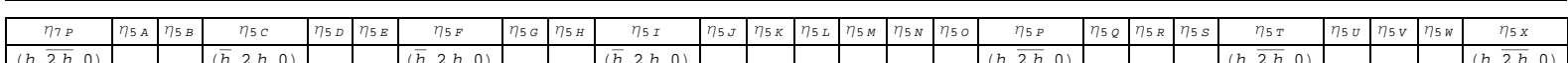

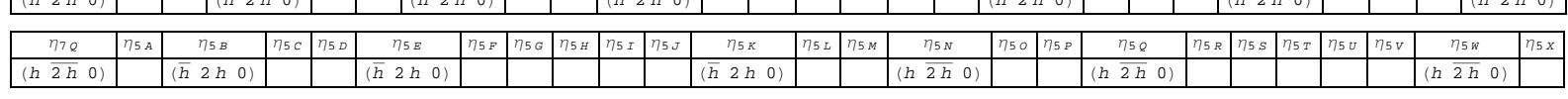

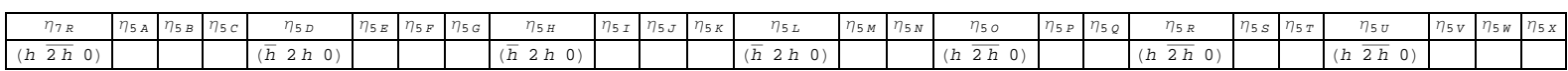

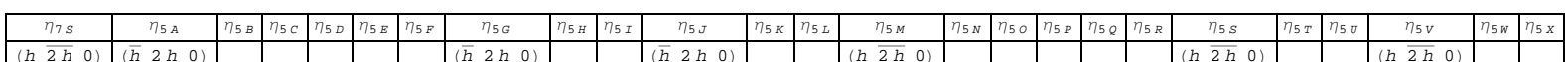

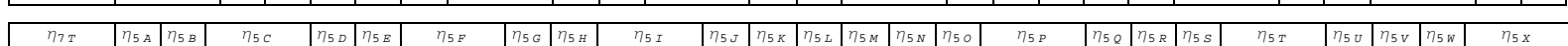

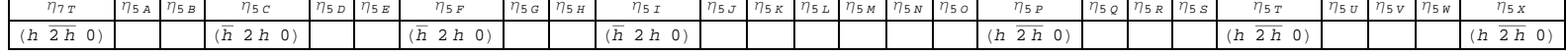

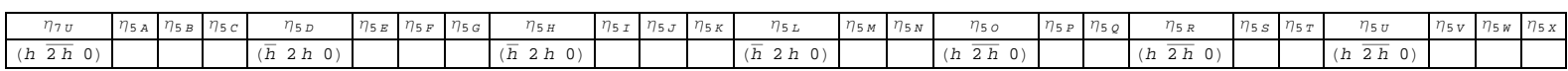

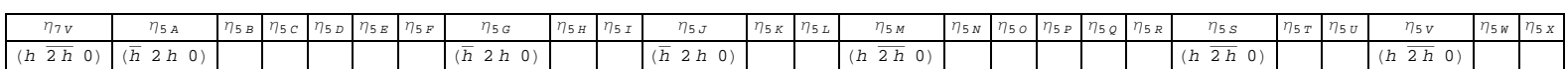

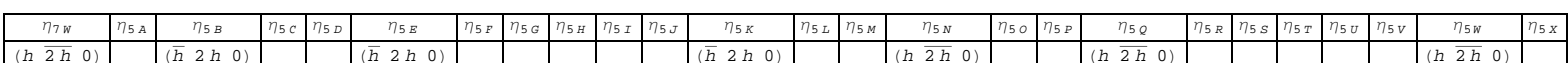

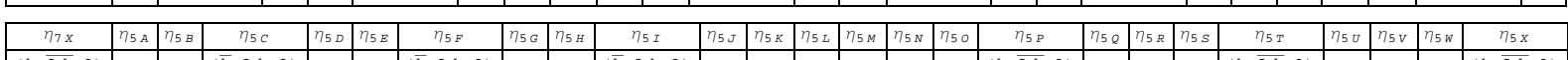

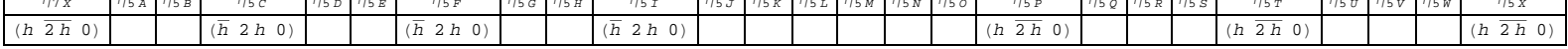


$\left(\eta_{7}, \eta_{6}\right)$

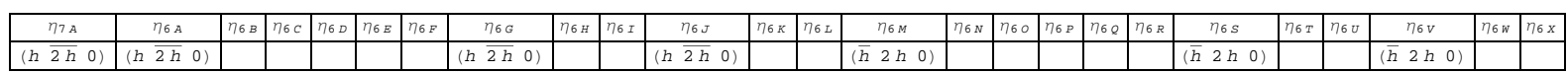

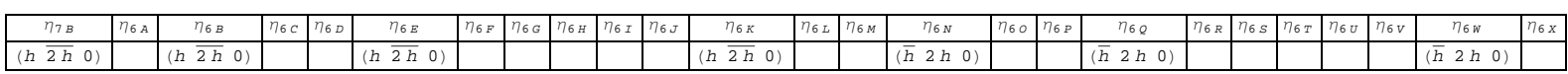

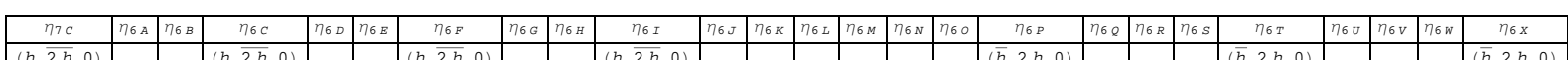

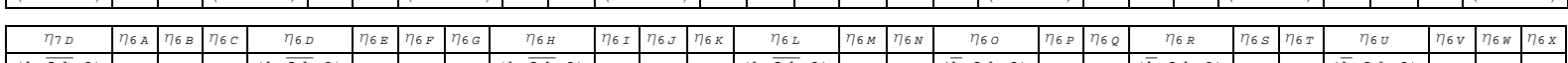

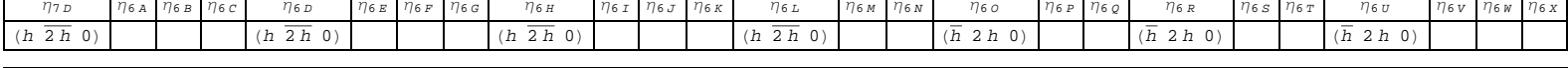

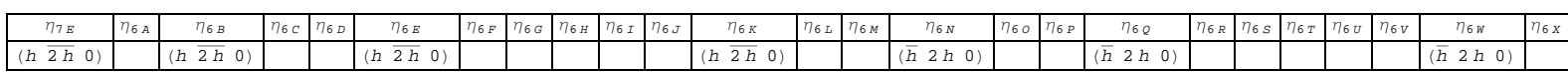

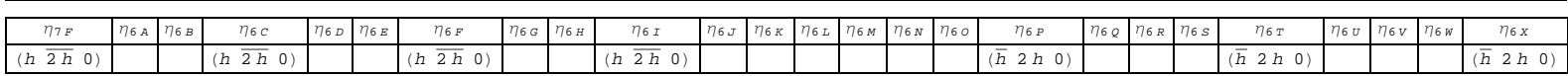

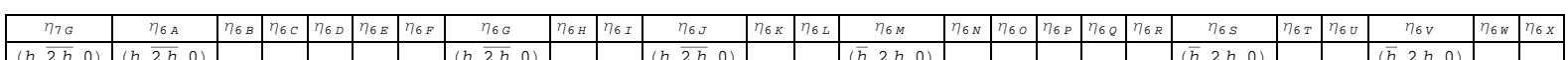

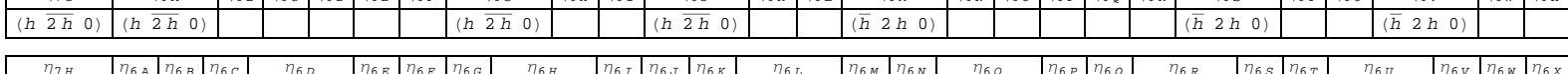

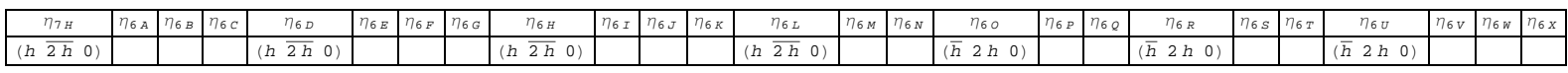

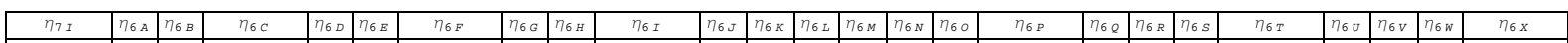
\begin{tabular}{|l|l|l|l|l|l|l|l|l|l|l|l|l|l|l|l|l|l|}
\hline$(h 2 h \theta)$ & $(h 2 h \theta)$ & & & $(h 2 h \theta)$ \\
\hline
\end{tabular}

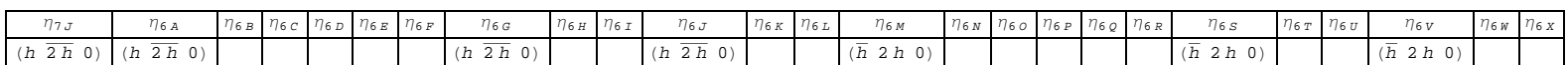

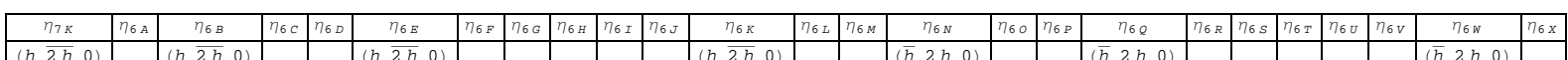

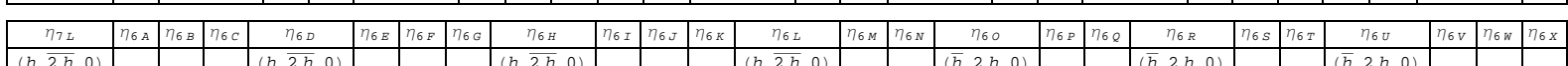

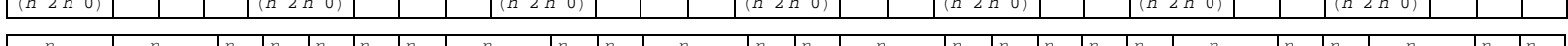

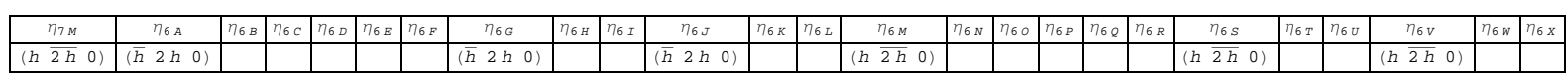

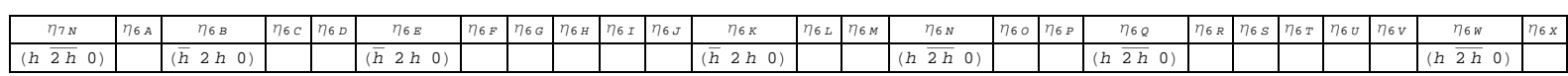

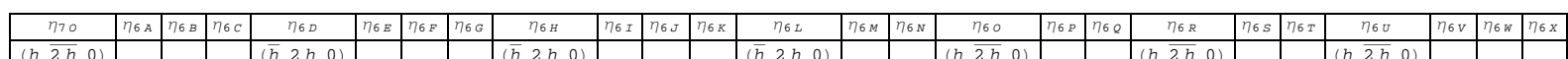

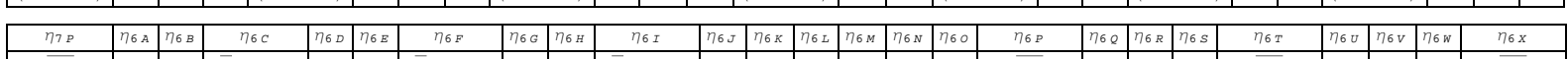

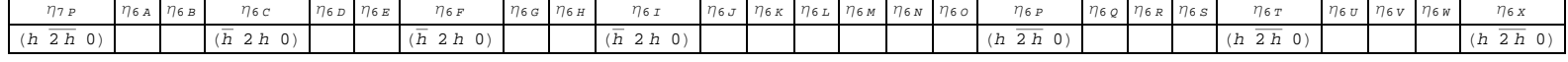

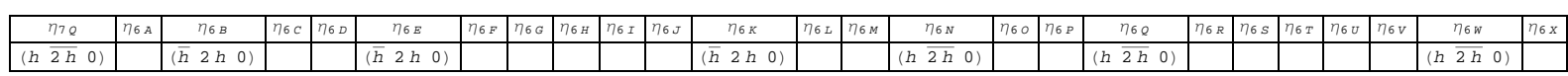

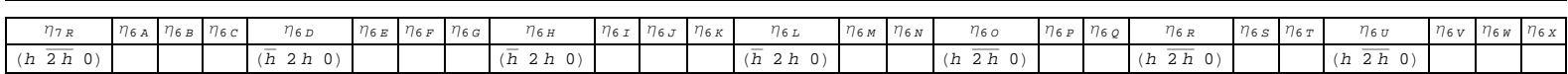

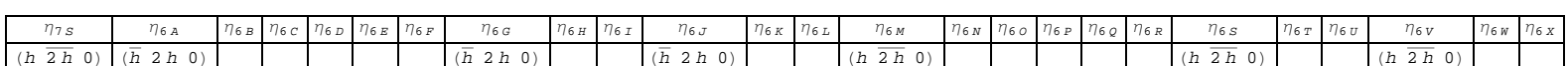

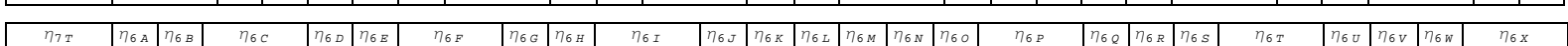

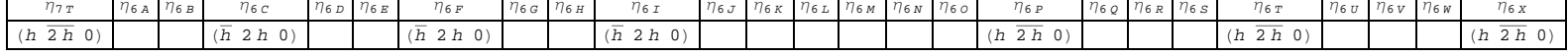

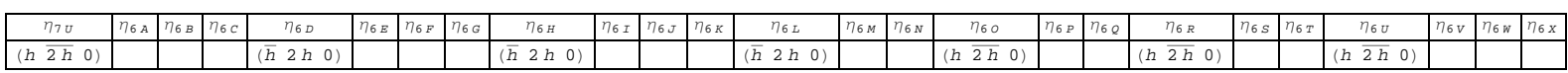

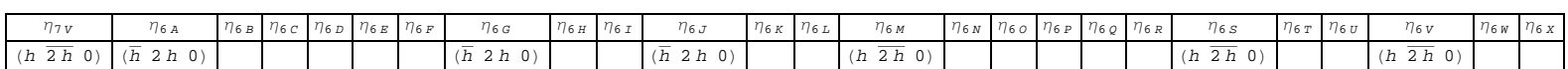

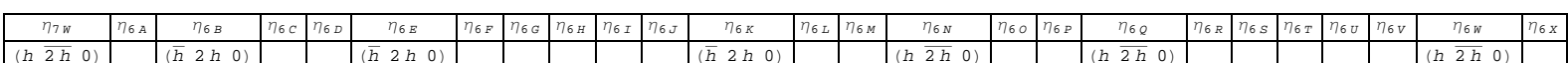

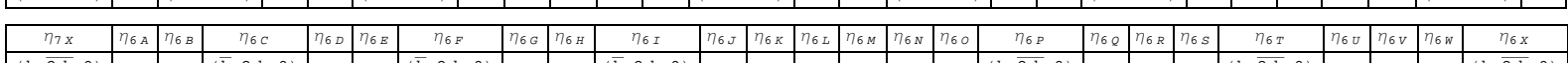

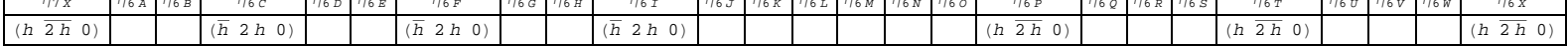


$\left(\eta_{8}, \eta_{2}\right)$

\begin{tabular}{|c|c|c|c|c|}
\hline$\eta_{8 A}$ & $\eta_{2 A}$ & $\eta_{2 B}$ & $\eta_{2 C}$ & $\eta_{2 D}$ \\
\hline$(h \overline{2 h} \Theta)$ & $(h \overline{2 h} \Theta)$ & & & \\
\hline
\end{tabular}

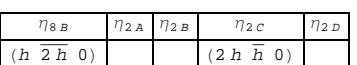

\begin{tabular}{|c|c|c|c|c|}
\hline$\eta_{8 C} C$ & $\eta_{2 A}$ & $\eta_{2 B}$ & $\eta_{2 C}$ & $\eta_{2 D}$ \\
\hline$(n \overline{2 h} \theta$ & & & & $(2 h \bar{h} \theta)$ \\
\hline
\end{tabular}

\begin{tabular}{|c|c|c|c|c|}
\hline$\eta_{80}$ & $\eta_{2 A}$ & $\eta_{2 B}$ & $\eta_{2 C}$ & $\eta_{2 D}$ \\
\hline$(h \overline{2 h} \theta)$ & & $(h \overline{2 h} \theta)$ & & \\
\hline
\end{tabular}

\begin{tabular}{|c|c|c|c|c|}
\hline$\eta_{8 E}$ & $\eta_{2 A}$ & $\eta_{28}$ & $\eta_{2 c}$ & $\eta_{20}$ \\
\hline$(h 2 h \theta)$ & & & $(h \bar{h} \theta)$ & \\
\hline
\end{tabular}

\begin{tabular}{|c|c|c|c|c|}
\hline$\eta_{8 F}$ & $\eta_{2 A}$ & $\eta_{2 B}$ & $\eta_{2 C}$ & $\eta_{2 D}$ \\
\hline$\langle h \overline{2} \boldsymbol{h} \theta)$ & & & & $(\bar{h} \hbar \theta)$ \\
\hline
\end{tabular}

\begin{tabular}{|l|l|l|l|l|}
\hline$\eta_{86}$ & $\eta_{2 \mathrm{~A}}$ & $\eta_{2 \mathrm{~B}}$ & $\eta_{22} \mathrm{C}$ & $\eta_{20} \mathrm{O}$ \\
\hline
\end{tabular}

\begin{tabular}{|c|c|c|c|c|}
\hline$\eta_{86}$ & $\eta_{2 A}$ & $\eta_{2 B}$ & $\eta_{2 C}$ & $\eta_{20}$ \\
\hline$(h \overline{2 \hbar} \theta)$ & $(h h \theta)$ & & & \\
\hline
\end{tabular}

\begin{tabular}{|c|c|c|c|c|}
\hline$\eta_{8 \mathrm{H}}$ & $\eta_{2 \mathrm{~A}}$ & $\eta_{2 \mathrm{~B}}$ & $\eta_{2 \mathrm{C}}$ & $\eta_{2 \mathrm{D}}$ \\
\hline$(n \overline{2 h} \theta$ & & $(\mathrm{h})$ & & \\
\hline
\end{tabular}

\begin{tabular}{|l|l|l|l|l|}
\hline$(h \overline{2 \hbar} \theta)$ & & $(h h \theta)$ & & \\
\hline
\end{tabular}

\begin{tabular}{|c|c|c|c|c|}
\hline$\eta_{8 I}$ & $\eta_{2 A}$ & $\eta_{2 B}$ & $\eta_{2 C}$ & $\eta_{2 D}$ \\
\hline$\left(h \begin{array}{ll}2 h & \theta\end{array}\right)$ & & & & $(h 2 h \theta)$ \\
\hline
\end{tabular}

\begin{tabular}{|c|c|c|c|c|}
\hline$\eta_{8} J$ & $\eta_{2 A}$ & $\eta_{2 B}$ & $\eta_{2 C} C$ & $\eta_{2 D}$ \\
\hline$(h \overline{2 h} \Theta)$ & $(\overline{2 h} h \Theta)$ & & & \\
\hline
\end{tabular}

\begin{tabular}{|c|c|c|c|c|}
\hline$\eta_{8 K} K$ & $\eta_{2 A} \mathrm{~A}$ & $\eta_{28} \mathrm{~B}$ & $\eta_{22} \mathrm{C}$ & $\eta_{20} \mathrm{O}$ \\
\hline$(h \overline{2 h} \Theta)$ & & & $(\bar{h} 2 h \Theta)$ & \\
\hline
\end{tabular}

\begin{tabular}{|c|c|c|c|c|}
\hline$\eta_{8 L}$ & $\eta_{2 A} A$ & $\eta_{2 B}$ & $\eta_{2 C} C$ & $\eta_{20}$ \\
\hline$(h \overline{2 h} \Theta)$ & & $(\overline{2 h} h \Theta)$ & & \\
\hline
\end{tabular}

\begin{tabular}{|c|c|c|c|c|}
\hline$\eta_{8 M}$ & $\eta_{2 A}$ & $\eta_{2 B}$ & $\eta_{2 C}$ & $\eta_{20}$ \\
\hline$(h \overline{2 h} \Theta)$ & $(\bar{h} 2 h \Theta)$ & & & \\
\hline
\end{tabular}

\begin{tabular}{|c|c|c|c|c|}
\hline$\eta_{8 \mathrm{~N}}$ & $\eta_{2 \mathrm{~A}}$ & $\eta_{2 \mathrm{~B}}$ & $\eta_{2 \mathrm{C}}$ & $\eta_{2 \mathrm{O}}$ \\
\hline$(h \overline{2 h} \Theta)$ & & & $(\overline{2 h} h \theta)$ & \\
\hline
\end{tabular}

\begin{tabular}{|c|c|c|c|c|}
\hline$\eta_{80}$ & $\eta_{2 A}$ & $\eta_{2 B}$ & $\eta_{2 C}$ & $\eta_{20}$ \\
\hline$(h \overline{2 h} \theta)$ & & $(\bar{h} 2 h \theta)$ & & \\
\hline
\end{tabular}

\begin{tabular}{|c|c|c|c|c|}
\hline$\eta_{8 P}$ & $\eta_{2 A}$ & $\eta_{2 B}$ & $\eta_{2 C}$ & $\eta_{2 D}$ \\
\hline$(h \overline{2 h} \Theta)$ & & & & $(\overline{2 h} h \theta)$ \\
\hline
\end{tabular}

\begin{tabular}{|c|c|c|c|c|}
\hline$\eta_{8 Q} Q$ & $\eta_{2 A}$ & $\eta_{2 B}$ & $\eta_{2 C}$ & $\eta_{2 D}$ \\
\hline$(h \overline{2 h} \Theta)$ & & & $(h \overline{2 h} \Theta)$ & \\
\hline
\end{tabular}

\begin{tabular}{|c|c|c|c|c|}
\hline$\eta_{8 R}$ & $\eta_{2 A} A$ & $\eta_{2 B}$ & $\eta_{2 C} C$ & $\eta_{2 D}$ \\
\hline$(h 2 h \theta)$ & & $(2 h \hbar \theta)$ & & \\
\hline
\end{tabular}

\begin{tabular}{|c|c|c|c|c|}
\hline$\eta_{8 S} S$ & $\eta_{2 A}$ & $\eta_{2 B}$ & $\eta_{2 C} C$ & $\eta_{2 D}$ \\
\hline$(h \overline{2 h} \Theta)$ & $(2 h \bar{h} \Theta)$ & & & \\
\hline
\end{tabular}

\begin{tabular}{|c|c|c|c|c|}
\hline$\eta_{8} T$ & $\eta_{2 A}$ & $\eta_{2 B}$ & $\eta_{2 C} C$ & $\eta_{2 D}$ \\
\hline \hline$h 2 h$ & & & & \\
\hline
\end{tabular}

\begin{tabular}{|l|l|l|l|}
\hline$(h \overline{2 h} \theta)$ & & & $(h \overline{2 h} \theta)$ \\
\hline
\end{tabular}

\begin{tabular}{|c|c|c|c|c|}
\hline$\eta_{8 U}$ & $\eta_{2 A}$ & $\eta_{2 B}$ & $\eta_{2 C}$ & $\eta_{20}$ \\
\hline$(h 2 h \theta)$ & & $(h \bar{h} \theta)$ & & \\
\hline
\end{tabular}

\begin{tabular}{|c|c|c|c|c|}
\hline$\eta_{8 \mathrm{~V}}$ & $\eta_{2 \mathrm{~A}}$ & $\eta_{2 B}$ & $\eta_{2 \mathrm{C}}$ & $\eta_{20}$ \\
\hline$(h 2 \mathrm{~h} \theta)$ & $($ h $\hbar \theta$ & & & \\
\hline
\end{tabular}

\begin{tabular}{|l|l|l|l|l|}
\hline$\eta_{8 \mathrm{~W}}$ & $\eta_{2 \mathrm{~A}}$ & $\eta_{2 \mathrm{~B}}$ & $\eta_{2 \mathrm{C}}$ & $\eta_{2 \mathrm{O}}$ \\
\hline
\end{tabular}

\begin{tabular}{|l|l|l|l|}
\hline (h $\overline{2 h} \theta)$ & & $(h h \theta)$ & \\
\hline
\end{tabular}

\begin{tabular}{|c|c|c|c|c|}
\hline$\eta_{8 X} x$ & $\eta_{2 A}$ & $\eta_{2 B}$ & $\eta_{22} c$ & $\eta_{22}$ \\
\hline$(h \overline{2 h} \theta)$ & & & & $(h h \theta)$ \\
\hline
\end{tabular} 
$\left(\eta_{8}, \eta_{11}\right)$

\begin{tabular}{|c|c|c|c|c|c|c|c|c|c|c|c|c|c|}
\hline$\eta_{8 A}$ & \multirow{2}{*}{\multicolumn{2}{|c|}{$\begin{array}{c}\eta_{11 \mathrm{~A}} \\
\bar{h} 2 \mathrm{~h} \Theta)\end{array}$}} & $\eta_{11 B}$ & $\eta_{11 c}$ & $\eta_{110}$ & $\eta_{111 \mathrm{E}}$ & $\eta_{11 F}$ & $\eta_{11 G}$ & $\eta_{11 H}$ & $\eta_{111}$ & $\frac{711 \mathrm{~J}}{712}$ & $\eta_{11 k}$ & $711 \angle$ \\
\hline$h \overline{2 h} \theta)$ & & & & & & & & & & & & & \\
\hline$\eta_{8 B}$ & $\eta_{11 \mathrm{~A}}$ & $\eta_{11}$ & & $\eta_{11 c}$ & $\eta_{110}$ & \begin{tabular}{|l|l|}
$\eta_{111}$ \\
\end{tabular} & $\eta_{11 F}$ & $\eta_{116}$ & $\eta_{11 \mathrm{H}}$ & $\eta_{11 I}$ & $\eta_{11 \mathrm{~J}}$ & $\eta_{11 K}$ & $\eta_{11 L}$ \\
\hline$\overline{h \overline{2 h} \Theta)}$ & & $(\bar{h} 21$ & & & & & & & & & & & \\
\hline$\eta_{88}$ & $\eta_{11 \mathrm{~A}}$ & $\eta_{11 B}$ & $\eta_{11}$ & & $\eta_{110}$ & \begin{tabular}{|l|}
$\eta_{11 E}$ \\
\end{tabular} & $\eta_{11 F}$ & $\eta_{116}$ & $\eta_{11 H}$ & \begin{tabular}{|l|}
$\eta_{11 I}$ \\
\end{tabular} & $\eta_{11 \mathrm{~J}}$ & \begin{tabular}{|l|l|}
$\eta_{11 K}$ \\
\end{tabular} & $\eta_{11 L}$ \\
\hline $\begin{array}{l}n \overline{2 h} \theta) \\
\end{array}$ & & & $\mid \begin{array}{ll}\begin{array}{ll}5 & 2\end{array} \mid \\
\end{array}$ & $\begin{array}{ll}h \theta & 0 \\
\end{array}$ & & & & & & & & & \\
\hline$\eta_{80}$ & $\eta_{11 \mathrm{~A}}$ & $\eta_{118}$ & $\eta_{11 c}$ & & 110 & $\eta_{111 E}$ & $\eta_{11 F}$ & $\eta_{116}$ & $\eta_{11 H}$ & $\eta_{11 I}$ & $\eta_{11 j}$ & $\mid \eta_{11 k}$ & $\eta_{11 L}$ \\
\hline$h \overline{2 h} \theta)$ & & & & $(\bar{h} 2$ & $\begin{array}{ll}h & 0 \\
\end{array}$ & & & & & & & & \\
\hline$\eta_{8 E}$ & $\eta_{11 \mathrm{~A}}$ & $7_{118}$ & $\eta_{11 c}$ & $\eta_{110}$ & $\eta_{1}$ & & $\eta_{11 F}$ & $\eta_{116}$ & \begin{tabular}{|l|l}
$\eta_{11 H}$ \\
\end{tabular} & $\eta_{11 I}$ & $\frac{711 \mathrm{~J}}{71}$ & $\eta_{11 k}$ & $11 L$ \\
\hline$h 2 h \theta)$ & & & & & \begin{tabular}{ll}
\multicolumn{1}{l}{2} & 2 \\
\end{tabular} & 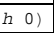 & & & & & & & \\
\hline$\eta_{8 F}$ & $\eta_{11 \mathrm{~A}}$ & $\eta_{11 B}$ & $\eta_{11 c}$ & $\eta_{110}$ & $\eta_{11 E}$ & $\eta_{1}$ & & $\eta_{11 \mathrm{G}}$ & $\eta_{11 H}$ & $\eta_{111}$ & $\eta_{11 \mathrm{~J}}$ & \begin{tabular}{|l|l|}
$\eta_{11 K}$ & \\
\end{tabular} & $\eta_{11 L}$ \\
\hline$h 2 h \theta)$ & & & & & & (h 2 & ho) & & & & & & \\
\hline$\eta_{8 G}$ & $\eta_{11 \mathrm{~A}}$ & $\eta_{11 \mathrm{~B}}$ & $\eta_{11 c}$ & $\eta_{11 D}$ & $\eta_{11 E}$ & $\eta_{11 F}$ & $\eta_{11}$ & & $\eta_{11 \mathrm{H}}$ & $\eta_{11 I}$ & $\eta_{11 \mathrm{~J}}$ & $\left|\eta_{11 K}\right|$ & $\eta_{111}$ \\
\hline h2h & & & & & & & \begin{tabular}{ll}
\multicolumn{1}{l}{} & 2 \\
\end{tabular} & $h \theta$ & & & & & \\
\hline $7_{8 \mathrm{H}}$ & $n_{11 \mathrm{~A}}$ & $\eta_{11 B}$ & $\eta_{11 c}$ & $\eta_{110}$ & $\eta_{11 \mathrm{E}}$ & $\eta_{11 F}$ & $\eta_{116}$ & $\eta_{13}$ & & $\eta_{11 I}$ & $\eta_{113}$ & $\overline{\eta_{11 K}}$ & $\eta_{11 L}$ \\
\hline$(h \overline{2 h} \theta)$ & & & & & & & & (h 2 & ho) & & & & \\
\hline$\eta_{8 I}$ & $\eta_{11 \mathrm{~A}}$ & $\eta_{11 B}$ & $\eta_{11 c}$ & $7_{110}$ & $\eta_{11 E}$ & $\eta_{11 F}$ & \begin{tabular}{|l|l|}
$\eta_{116}$ \\
\end{tabular} & $\eta_{11 \mathrm{H}}$ & $\eta_{1}$ & & $\eta_{11 \mathrm{~J}}$ & $\begin{array}{ll}\eta_{11 K} \\
\end{array}$ & $\eta_{11 L}$ \\
\hline$(h \overline{2 h} \theta)$ & & & & & & & & & \begin{tabular}{ll}
\multicolumn{1}{l}{$h_{2}$} \\
\end{tabular} & 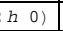 & & & \\
\hline $78 \mathrm{~J}$ & $\eta_{11 \mathrm{~A}}$ & $\eta_{11 B}$ & $\eta_{11 c}$ & $\eta_{11 D}$ & $\eta_{11 E}$ & $\eta_{11 F}$ & $\eta_{116}$ & $\eta_{11 \mathrm{H}}$ & $\eta_{11 I}$ & $\eta_{11}$ & & $\eta_{11 K}$ & $\eta_{11 L}$ \\
\hline$(h \overline{2 h} \quad \theta)$ & & & & & & & & & & (h 2 & $h \theta$ & & \\
\hline $78 \mathrm{~K}$ & $\eta_{11 A}$ & $\eta_{11 B}$ & $\eta_{11 c}$ & $\eta_{110}$ & $\eta_{111 \mathrm{E}}$ & $\mid \eta_{11 F}$ & $\eta_{116}$ & $\eta_{11 H}$ & $\eta_{11 I}$ & $\eta_{11 \mathrm{~J}}$ & $\eta_{1}$ & & $\eta_{11 L}$ \\
\hline$(h \overline{2 h} \theta)$ & & & & & & & & & & & $\mid \begin{array}{ll}\bar{h} & 2 \\
\end{array}$ & $2 h \theta)$ & \\
\hline $78 \mathrm{~L}$ & $\eta_{11 A}$ & $\eta_{11 B}$ & $\eta_{11 c}$ & $\eta_{110}$ & $\eta_{11 \mathrm{E}}$ & $\eta_{11 F}$ & $\mid \eta_{116}$ & $\eta_{11 \mathrm{H}}$ & $\eta_{11 I}$ & \begin{tabular}{ll|}
$\eta_{11} \mathrm{~s}$ & \\
\end{tabular} & $\eta_{11 k}$ & $\eta_{1}$ & \\
\hline$(h \overline{2 h} \theta)$ & & & & & & & & & & & & & $2 h \theta)$ \\
\hline$\eta_{8 M}$ & $\eta_{11}$ & & \begin{tabular}{|l|}
$\eta_{1118}$ \\
\end{tabular} & $\eta_{11 c}$ & $\eta_{110}$ & $\eta_{11 E}$ & $\eta_{11 F}$ & $\eta_{116}$ & $\eta_{11 H}$ & $\eta_{111}$ & $\eta_{11 \mathrm{~J}}$ & \begin{tabular}{|l|l|}
$\eta_{11 k}$ & \\
\end{tabular} & $\eta_{11 L}$ \\
\hline$(h \overline{2 h} \theta)$ & (h) $\overline{2}$ & (h⿱一𫝀) & & & & & & & & & & & \\
\hline$n_{88}$ & $\eta_{11 \mathrm{~A}}$ & $\eta_{11}$ & & $\eta_{11 c}$ & $\eta_{110}$ & $\eta_{11 \mathrm{E}}$ & $\eta_{11 F}$ & $\eta_{116}$ & $\eta_{11 H}$ & $\eta_{11 \mathrm{I}}$ & $\eta_{11 \mathrm{~J}}$ & \begin{tabular}{|l|l|}
$\eta_{11 K}$ \\
\end{tabular} & $\eta_{11 L}$ \\
\hline$h \overline{2 h} \theta)$ & & $(h \overline{2}$ & $\begin{array}{l}\bar{h} \text { e) } \\
\end{array}$ & & & & & & & & & & \\
\hline$\eta_{80}$ & $\eta_{11 \mathrm{~A}}$ & $\mid \eta_{11 B}$ & $\eta_{11 c}$ & & 110 & $\eta_{111 E}$ & $\eta_{11 F}$ & $\eta_{116}$ & $\eta_{11 \mathrm{H}}$ & $\eta_{11 I}$ & $\eta_{11 \mathrm{~J}}$ & $\eta_{11 K}$ & $\eta_{114}$ \\
\hline$(h \overline{2 h} \theta)$ & & & & $\langle h \overline{\overline{2}}$ & $2 \overline{20}$ & & & & & & & & \\
\hline$\eta_{8 P}$ & $\eta_{11 \mathrm{~A}}$ & $\eta_{11 B}$ & $\eta_{11}$ & & $\eta_{110}$ & $\eta_{11 E}$ & $\eta_{11 F}$ & $\eta_{116}$ & $\eta_{11 H}$ & $\eta_{111}$ & $\eta_{111}$ & \begin{tabular}{|l|l|}
$\eta_{11 K}$ & \\
\end{tabular} & $\eta_{11 L}$ \\
\hline$(h \overline{2 h} \theta)$ & & & $(h \overline{21}$ & $\begin{array}{ll}\bar{h} 0) \\
\end{array}$ & & & & & & & & & \\
\hline$\eta_{8 Q}$ & \begin{tabular}{|l|l|}
$\eta_{11 A}$ & \\
\end{tabular} & $\eta_{11 B}$ & $\eta_{11 c}$ & $\eta_{110}$ & $\eta_{11 E}$ & $\eta_{11 F}$ & $\eta_{116}$ & $\eta_{11 H}$ & $\eta_{11 I}$ & $\eta_{11 \mathrm{~J}}$ & $\eta_{1}$ & & $\eta_{11 L}$ \\
\hline$(h \overline{2 h} \theta)$ & & & & & & & & & & & $(h \overline{2}$ & $2 h \quad 0)$ & \\
\hline$\eta_{8 R}$ & \begin{tabular}{|l|l|}
$\eta_{11 A}$ \\
\end{tabular} & $\eta_{11 B}$ & $\eta_{11 c}$ & $\eta_{110}$ & $\eta_{11 E}$ & $\eta_{11 F}$ & $\eta_{116}$ & $\eta_{11 \mathrm{H}}$ & $\eta_{11 I}$ & $\eta_{11 \mathrm{~J}}$ & $\eta_{11 K}$ & $\eta_{1}$ & \\
\hline $\begin{array}{ll}25 \theta) \\
\end{array}$ & & & & & & & & & & & & (h) & $2 h$ ब) \\
\hline$\eta_{8 s}$ & $\eta_{11 \mathrm{~A}}$ & $\eta_{11 B}$ & $\eta_{11 c}$ & $\eta_{110}$ & $\eta_{11 E}$ & $\eta_{11 F}$ & $\eta_{116}$ & $\eta_{11 \mathrm{H}}$ & $\eta_{11 I}$ & $\eta_{11}$ & 111 & $\eta_{11 K}$ & $\eta_{11 L}$ \\
\hline$(h \overline{2 h} \theta)$ & & & & & & & & & & & $250)$ & & \\
\hline$\eta_{81}$ & $\eta_{11 A}$ & $\eta_{11 B}$ & $\eta_{11 c}$ & $\eta_{110}$ & $\eta_{11 E}$ & $\eta_{11 F}$ & $\eta_{116}$ & $\eta_{11 \mathrm{H}}$ & $\eta_{1}$ & & $\eta_{11 j}$ & \begin{tabular}{|l|l|}
$\eta_{11 k}$ \\
\end{tabular} & $\eta_{11 L}$ \\
\hline$(h \overline{2 h} \theta)$ & & & & & & & & & $\begin{array}{ll}l & \overline{2} \\
\end{array}$ & $250)$ & & & \\
\hline$\eta_{88}$ & $\eta_{11 A}$ & $\eta_{11 B}$ & $\eta_{11 c}$ & $\eta_{110}$ & $\eta_{11 E}$ & $\eta_{11 F}$ & $\eta_{116}$ & $\eta_{12}$ & $11 H$ & $\eta_{111}$ & $\eta_{11 \mathrm{~J}}$ & $\begin{array}{ll}\eta_{11 k} \\
\end{array}$ & $\eta_{11 L}$ \\
\hline 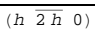 & & & & & & & & $\left\langle\begin{array}{ll}h & 2\end{array}\right.$ & $2 h \quad 0)$ & & & & \\
\hline$\eta_{88} \mathrm{~V}$ & $\eta_{11 A}$ & $\eta_{11 B}$ & $\eta_{11 c}$ & $\eta_{110}$ & $\eta_{11 E}$ & $\eta_{11 F}$ & $\eta_{11}$ & 116 & $\eta_{11 \mathrm{H}}$ & $\eta_{11 I}$ & $\eta_{11 \mathrm{~J}}$ & $\eta_{11 K}$ & $\eta_{11 L}$ \\
\hline$(h 2 h \theta)$ & & & & & & & $\left(\begin{array}{ll}h 2 \\
\end{array}\right.$ & h o) & & & & & \\
\hline$\eta_{8 \mathrm{w}}$ & $\eta_{11 A}$ & $\eta_{11 B}$ & $n_{11 c}$ & $\eta_{110}$ & $\overline{\eta_{1}}$ & & $n_{11 F}$ & $\eta_{116}$ & $\eta_{11 \mathrm{H}}$ & $\eta_{111}$ & $\eta_{110}$ & $\eta_{11 k}$ & $\eta_{11 L}$ \\
\hline$(h \overline{2 h} \theta)$ & & & & & $\langle h \overline{2}$ & & & & & & & & \\
\hline $78 x$ & $7_{11 A}$ & $\eta_{11 B}$ & $\eta_{11 c}$ & 7110 & $\eta_{111 E}$ & $\eta_{1}$ & & \begin{tabular}{|l|l|}
$\eta_{116}$ \\
\end{tabular} & $\eta_{11 \mathrm{H}}$ & $\eta_{111}$ & $\eta_{111}$ & $\eta_{11 K}$ & $\eta_{11 L}$ \\
\hline$(h \overline{2 h} \theta)$ & & & & & & $\langle h \overline{2}$ & h 0$)$ & & & & & & \\
\hline
\end{tabular}


$\left(\eta_{9}, \eta_{\mathrm{I}}\right)$

\begin{tabular}{|c|c|c|c|c|c|c|c|}
\hline$\eta_{9 A}$ & \multicolumn{3}{|c|}{\begin{tabular}{c|c}
$\eta_{1 A}$ & $\eta_{1 B}$ \\
\end{tabular}} & $\eta_{1 c}$ & $\eta_{10}$ & $\eta_{1 E}$ & $\eta_{1 F}$ \\
\hline$\left(\begin{array}{lll}(0 & 0 & 1\end{array}\right)$ & 100 & & & & & & \\
\hline$\eta_{9 B}$ & $\eta_{1 A}$ & & & $\eta_{1 c}$ & $\eta_{10}$ & $\eta_{1 E}$ & $\eta_{1 F}$ \\
\hline$\left(\begin{array}{lll}0 & 0 & 1\end{array}\right)$ & & $(\theta)$ & 1) & & & & \\
\hline$\eta_{9 c}$ & \begin{tabular}{ll|}
$\eta_{1 A}$ \\
\end{tabular} & $\eta_{1 B}$ & & & $\eta_{10}$ & $\eta_{1 E}$ & $\eta_{1 F}$ \\
\hline$\left(\begin{array}{lll}0 & 0 & 1\end{array}\right)$ & & & $(\theta)$ & 1) & & & \\
\hline $\begin{array}{l}\frac{790}{(001)} \\
(001)\end{array}$ & $\eta_{1 \mathrm{~A}}$ & $\eta_{1 B}$ & $n_{1 c}$ & & .0 & $\eta_{1 E}$ & $\eta_{1 F}$ \\
\hline$\eta_{9 \mathrm{E}}$ & \begin{tabular}{|l|}
$\eta_{1 A}$ \\
\end{tabular} & $\eta_{1 B}$ & $\eta_{1 c}$ & $\eta_{10}$ & $\eta_{1}$ & & $\eta_{1 F}$ \\
\hline$(001)$ & & & & & & $01)$ & \\
\hline$\eta_{9 F}$ & $\eta_{1 A}$ & $\eta_{1 B}$ & $\eta_{1 c}$ & $\eta_{10}$ & $\eta_{1 E}$ & & \\
\hline$\left(\begin{array}{lll}0 & 0 & 1\end{array}\right)$ & & & & & & & 1) \\
\hline
\end{tabular}

$\left(\eta_{9}, \eta_{2}\right)$

\begin{tabular}{|c|c|c|c|c|c|}
\hline$\eta_{9 \mathrm{~A}}$ & 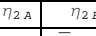 & $B$ & $\eta_{2 c}$ & $n_{2}$ & \\
\hline$\left(\begin{array}{llll}h & \odot & \theta\end{array}\right)$ & \begin{tabular}{l|l} 
& $(\bar{h} \theta$ \\
\end{tabular} & & & & (0) \\
\hline$\frac{\eta_{9 B}}{(h \odot \theta)}$ & \begin{tabular}{c|}
$\eta_{2 A}$ \\
$(h \odot \theta)$
\end{tabular} & $\overline{728}_{28}$ & \multicolumn{3}{|c|}{ 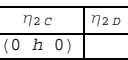 } \\
\hline$\eta_{9 c}$ & \multirow{2}{*}{ 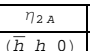 } & \multirow{2}{*}{\multicolumn{2}{|c|}{\begin{tabular}{|c|}
$\eta_{2 B}$ \\
$h \bar{h} \theta)$
\end{tabular}}} & \multirow{2}{*}{\multicolumn{2}{|c|}{\begin{tabular}{|l|l|}
$\eta_{2 c}$ & $\eta_{20}$ \\
\end{tabular}}} \\
\hline$\left(\begin{array}{lll}h & \odot & \theta\end{array}\right)$ & & & & & \\
\hline$\eta_{9 D}$ & \begin{tabular}{l|l|}
$\eta_{2 A}$ & $\eta_{2 B}$
\end{tabular} & $\eta_{2}$ & & & \\
\hline$\left(\begin{array}{lll}h & 0 & 0\end{array}\right)$ & & & (日) & & (0) \\
\hline $\begin{array}{l}\frac{\eta_{9 E}}{(h \theta \theta)} \\
(h \theta 0)\end{array}$ & \begin{tabular}{l|l}
$\eta_{2 A}$ & $\eta_{2 E}$ \\
$(\theta \quad h$
\end{tabular} & B & & & $7_{20}$ \\
\hline $\begin{array}{l}\eta_{9 F} \\
h \theta \theta \theta\end{array}$ & \begin{tabular}{|c|}
$\eta_{2 A}$ \\
$(\theta h \theta)$
\end{tabular} & $\eta_{28}$ & $\eta_{2 c}$ & $\frac{\eta_{2}}{40}$ & 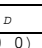 \\
\hline
\end{tabular}

$\left(\eta_{9}, \eta_{4}\right)$

\begin{tabular}{|c|c|c|c|c|c|c|c|c|c|c|c|c|c|c|}
\hline$\eta_{9 A}$ & $\eta_{4}$ & & $n_{4}$ & & $\eta_{4 c}$ & $\eta_{40}$ & $\eta_{4 E}$ & $\eta_{4 F}$ & $\eta_{46}$ & $7_{4 H}$ & $\eta_{4 I}$ & $74 \mathrm{~J}$ & $\eta_{4 k}$ & $7_{44}$ \\
\hline$\left(\begin{array}{llll}0 & 1\end{array}\right.$ & $\begin{array}{lll}\theta 0 \\
\end{array}$ & & $\angle \theta 0$ & & & & & & & & & & & \\
\hline$\eta_{9 B}$ & $\eta_{4 A}$ & $\eta_{48}$ & $\eta_{4}$ & & & & $7_{4 \mathrm{E}}$ & $\eta_{4 F}$ & $7_{4 G}$ & $\eta_{4 \mathrm{H}}$ & $\eta_{4 I}$ & $\eta_{44}$ & $\eta_{4 K}$ & $7_{4}$ \\
\hline$\left(\begin{array}{llll}0 & 0 & 1\end{array}\right)$ & & & 100 & 1) & & 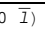 & & & & & & & & \\
\hline$\eta_{9 c}$ & $\eta^{\prime}$ & $\eta_{4 B}$ & $\eta_{4 c}$ & $\eta_{40}$ & & & $\eta_{4}$ & & $\eta_{46}$ & $7_{44}$ & $\eta_{41}$ & $\eta_{43}$ & ${ }^{\eta_{4 k}}$ & $\eta_{4 L}$ \\
\hline$\left(\begin{array}{llll}0 & 0\end{array}\right)$ & & & & & $(\theta$ & $01)$ & 100 & & & & & & & \\
\hline$\eta_{90}$ & $\eta_{4 \mathrm{~A}}$ & $\eta_{48}$ & $\eta_{4 c}$ & $\eta_{40}$ & $n_{4 E}$ & $\eta_{4 F}$ & $\eta_{4}^{4}$ & & $\eta_{4}$ & & $\eta_{4 I}$ & $\eta_{43}$ & $n_{44 K}$ & 1744 \\
\hline$\left(\begin{array}{llll}0 & 1\end{array}\right)$ & & & & & & & \begin{tabular}{|l|l}
$1 \theta 0$ \\
\end{tabular} & (1) & \begin{tabular}{|l|}
10 \\
\end{tabular} & (1) & & & & \\
\hline$\eta_{9 \mathrm{~F}}$ & $\eta_{4 \mathrm{~A}}$ & $77_{4 B}$ & $\eta_{4 c}$ & $77_{40}$ & $\begin{array}{l}77_{46} \\
\end{array}$ & $74 \mathrm{~F}$ & $77_{4 G}$ & $\eta_{44}$ & $\eta$ & & & $74 \mathrm{~J}$ & $\begin{array}{l}74 k \\
\end{array}$ & $\eta_{4 L}$ \\
\hline $\begin{array}{llll}0 & 0\end{array}$ & & & & & & & & & & $\begin{array}{lll}0 & 11 \\
\end{array}$ & & $\theta \bar{I})$ & & \\
\hline$\eta_{9 F}$ & $\eta_{4 \mathrm{~A}}$ & $74 B$ & $\eta_{4 c}$ & $7_{4 D}$ & 774 & $74 \mathrm{~F}$ & \begin{tabular}{|l}
$77 G$ \\
7
\end{tabular} & $\eta_{44}$ & $74 I$ & $7_{44} \mathrm{~J}$ & & $74 K$ & & \\
\hline$(001)$ & & & & & & & & & & & & $01)$ & & $\overline{0 \bar{I}}$ \\
\hline
\end{tabular}

$\left(\eta_{9}, \eta_{11}\right)$

\begin{tabular}{|c|c|c|c|c|c|c|c|c|c|c|c|c|c|c|}
\hline$\eta_{9 A}$ & $\eta_{111}$ & & $\eta_{11 \mathrm{~B}}$ & $\eta_{11}$ & \begin{tabular}{l|l}
11 & $\eta_{11}$ \\
\end{tabular} & \begin{tabular}{l|l}
10 & $\eta_{11}$ \\
\end{tabular} & \begin{tabular}{l|l|l}
$11 E$ & $\eta_{1}$ \\
\end{tabular} & \begin{tabular}{l|l}
$111 F$ & $\eta_{11}$ \\
\end{tabular} & \begin{tabular}{l|l}
$\eta_{116}$ & $\eta_{11}$ \\
\end{tabular} & $\eta_{11 \mathrm{H}}$ & $\eta_{11 I}$ & $711 \mathrm{~J}$ & $\eta_{111 k}$ & $\eta_{11 L}$ \\
\hline$\left(\begin{array}{lll}(0) & 1\end{array}\right)$ & $\left(\begin{array}{lll}\theta & 0 \\
\end{array}\right.$ & & 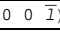 & & & & & & & & & & & \\
\hline$\eta_{9 B}$ & $\eta_{11 A}$ & $\eta_{11 B}$ & $\eta_{11 c}$ & & $\eta_{110}$ & $\eta_{11}$ & \begin{tabular}{l|l}
$11 E$ & $\eta_{1}$ \\
\end{tabular} & \begin{tabular}{l|l}
$111 F$ & $\eta_{11}$ \\
\end{tabular} & \begin{tabular}{ll|l}
$\eta_{116}$ & $\eta_{11}$ \\
\end{tabular} & $\begin{array}{l}\eta_{11 H} \\
\eta\end{array}$ & $\eta_{11 I} \mid$ & $\eta_{11 \mathrm{~J}}$ & $\eta_{11 K}$ & $n_{11 L}$ \\
\hline $\begin{array}{lll}0 & 0 & 1)\end{array}$ & & & $\begin{array}{lll}(\theta) & \theta \\
\end{array}$ & 1) & $\begin{array}{llll}(\theta) & 0 & 1\end{array}$ & & & & & & & & & \\
\hline$\eta_{9 c}$ & $\eta_{11 A}$ & $\eta_{11 B}$ & $\mid$\begin{tabular}{|l|}
$\eta_{11 c}$ \\
\end{tabular} & $\eta_{110}$ & $\eta_{11}$ & & $\eta_{11 \mathrm{~F}}$ & & \begin{tabular}{ll|}
$\eta_{116}$ & $\eta_{11}$
\end{tabular} & $\eta_{11 H} \eta$ & $\eta_{11 I}$ & $\eta_{111}$ & $\eta_{11 \kappa}$ & $\eta_{11 L}$ \\
\hline $\begin{array}{lll}0 & 0 & 1) \\
\end{array}$ & & & & & 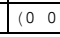 & \begin{tabular}{l|l} 
P1) \\
\end{tabular} & $(\theta)$ & & & & & & & \\
\hline 790 & $\eta_{11 \mathrm{~A}}$ & $\bar{\eta}_{11 B}$ & \begin{tabular}{|l|}
$\eta_{111}$ \\
\end{tabular} & $\eta_{110}$ & $\eta_{11 \mathrm{E}}$ & $\eta_{11 F}$ & $\eta_{11}$ & 116 & $\eta_{11 H}$ & $1 \mathrm{H}$ & $\eta_{11 I}$ & $\eta_{11} \mathrm{~J}$ & $\eta_{111 \kappa}$ & $\eta_{11 L}$ \\
\hline$\left(\begin{array}{lll}0 & 0 & 1\end{array}\right)$ & & & & & & & $(\theta)$ & \begin{tabular}{ll|l}
0 & $1)$ \\
\end{tabular} & $\begin{array}{lll}\theta & \theta & 1 \\
\end{array}$ & $\bar{I})$ & & & & \\
\hline$\eta_{9 E}$ & \begin{tabular}{|l|l|}
$\eta_{11 \mathrm{~A}}$ \\
\end{tabular} & $\eta_{11 B}$ & \begin{tabular}{|l|}
$\eta_{11 c}$ \\
\end{tabular} & $\eta_{110}$ & $\eta_{11 E}$ & $\eta_{11 F}$ & $\begin{array}{l}=\eta_{11 G} \\
\end{array}$ & $\begin{array}{ll}6 & \eta_{11 H} \\
\end{array}$ & & 7111 & & $11 \mathrm{~J}$ & $\eta_{111 K}$ & $\eta_{11 L}$ \\
\hline$\left(\begin{array}{lll}0 & 0 & 1\end{array}\right)$ & & & & & & & & & & $\begin{array}{lll}\theta & 1) \\
\end{array}$ & & $\theta \bar{I})$ & & \\
\hline$\eta_{9 F}$ & \begin{tabular}{|l|}
$\eta_{11 A}$ \\
\end{tabular} & $\eta_{11 B}$ & $\eta_{11 c}$ & $\eta_{110}$ & $\eta_{11 E}$ & $\eta_{11 F}$ & $\begin{array}{l}\eta_{116} \\
\end{array}$ & $\begin{array}{ll}6 & \eta_{111 \mathrm{H}}\end{array}$ & \begin{tabular}{l|l}
$H$ & $\eta_{11 I}$
\end{tabular} & \begin{tabular}{l|l|l}
11 & $\eta_{111}$
\end{tabular} & $1 \mathrm{~J}$ & $\eta_{11 K}$ & & $111 \mathrm{~L}$ \\
\hline$\left(\begin{array}{lll}0 & \theta & 1\end{array}\right)$ & & & & & & & & & & & & $0 \quad 0 \quad 1)$ & & $\theta \bar{I})$ \\
\hline
\end{tabular}

\section{$\left(\eta_{9}, \eta_{12}\right)$}

\begin{tabular}{|c|c|c|c|c|c|c|c|c|c|c|c|c|c|c|c|c|}
\hline$\eta_{9 \mathrm{~A}}$ & $\eta_{12 A}$ & $\eta_{128}$ & $\eta_{120}$ & & $\eta_{12 \mathrm{D}}$ & & $12 \mathrm{E}$ & $\eta_{12 F}$ & $\eta_{126}$ & $\eta_{12 \mathrm{H}}$ & \begin{tabular}{r|r} 
& $\eta_{12}$ \\
\end{tabular} & & \multirow{2}{*}{\multicolumn{2}{|c|}{\begin{tabular}{|c|}
$\eta_{12 J}$ \\
$(h h \theta)$
\end{tabular}}} & $\eta_{12 k}$ & $\eta_{12 L}$ \\
\hline$(h 2 h \theta)$ & & & $(h h$ & & $(\bar{h} \bar{C}$ & & & & & & 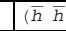 & 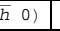 & & & & \\
\hline$\eta_{9 B}$ & $\eta_{12 A}$ & $\eta_{128}$ & $\bar{\eta}_{120}$ & & $\eta_{120}$ & & $\mid 12 E$ & $\eta_{12 F}$ & $\eta_{126}$ & $\eta_{12 H}$ & $\eta_{12}$ & & & $12 \mathrm{~J}$ & $\eta_{12 k}$ & $7_{12 L}$ \\
\hline$(h \overline{2 h} \theta)$ & & & (5 万 & & $\begin{array}{ll}h h \\
\end{array}$ & & & & & & \begin{tabular}{|l|l|}
$k h$ \\
\end{tabular} & 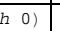 & & $\overline{\hbar \hbar \theta)}$ & & \\
\hline$\eta_{9 c}$ & $\eta_{12}$ & & $\eta_{12 B}$ & & $12 c \mid \eta_{1}$ & & $\mid 12 E$ & $\eta_{12 F}$ & $\eta_{12}$ & & $\eta_{12 H}$ & & 712 I & $\eta_{122}$ & $\eta_{12 K}$ & 47 \\
\hline$h \overline{2 h \theta)}$ & $\overline{k n} \bar{h}$ & & $(h h \theta$ & & & & & & $\langle\mathrm{hh}$ & (0) & $(\bar{h} \bar{h} \theta$ & & & & & \\
\hline$\eta_{90}$ & $\eta_{12}$ & & $\eta_{12 B}$ & $\eta_{12}$ & \begin{tabular}{l|l}
$12 c$ & $\eta_{1}$
\end{tabular} & & $\mid 12 \mathrm{E}$ & $\eta_{12 \mathrm{~F}}$ & $\eta_{12}$ & & $\eta_{12 H}$ & & $\mid 712 I$ & $\eta_{12 J}$ & $\eta_{12 k}$ & (र) $\eta_{12 L}$ \\
\hline$(h \overline{2 h} \theta\rangle$ & (hh & (0) & $(\bar{h} \bar{h} \theta$ & & & & & & $\mid \bar{h} \bar{h}$ & (70) & $(h h \theta$ & & & & & \\
\hline$\eta_{9 E}$ & $712 A$ & $\eta_{128}$ & $\eta_{12 c}$ & $\eta_{120}$ & $\eta_{12}$ & & & $12 F$ & 7126 & $\eta_{12 H}$ & $\begin{array}{ll}4 \eta_{12 I} \\
\end{array}$ & \begin{tabular}{|l|l|l}
$72 \mathrm{~J}$ \\
\end{tabular} & & $\eta_{12 k}$ & & $\eta_{12 L}$ \\
\hline$\langle h \overline{2 h} \theta\rangle$ & & & & & $\langle\bar{h} \bar{h}$ & & & $h 0)$ & & & & & & $h \quad h \quad 0$ & & $\bar{h} \theta$ \\
\hline$\eta_{9 F}$ & $\eta_{12 A}$ & $\eta_{128}$ & $\eta_{12 c}$ & $\overline{\eta_{120}}$ & $\eta_{12}$ & & & $12 F$ & $\eta_{126}$ & $\eta_{12 H}$ & $4 \eta_{12 I}$ & 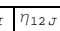 & & $\eta_{12 \mathrm{~K}}$ & & $\overline{712 L}$ \\
\hline$\langle(h \overline{2 h} \theta\rangle$ & & & & & $\langle\mathrm{hr}$ & & & $\bar{h} \theta)$ & & & & & & $\bar{h} \bar{h} \theta$ & & $h \theta$ \\
\hline
\end{tabular}


$\left(\eta_{10}, \eta_{2}\right)$

\begin{tabular}{|c|c|c|c|c|c|}
\hline$\eta_{10 \mathrm{~A}}$ & \multirow{2}{*}{\multicolumn{2}{|c|}{\begin{tabular}{|c|}
$\eta_{2 A}$ \\
$\left(\begin{array}{llll}\theta & 0\end{array}\right)$ \\
\end{tabular}}} & \multirow[t]{2}{*}{$\eta_{2 B}$} & $\eta_{2 c}$ & \multirow[t]{2}{*}{720} \\
\hline $\begin{array}{llll}0 & 1 \text { 1) }\end{array}$ & & & & & \\
\hline$\eta_{10 B}$ & $\eta_{2 A}$ & $\eta_{2 B}$ & & & $\eta_{2 c}$ \\
\hline$\left(\begin{array}{lll}0 & 0 & 1\end{array}\right)$ & & & $(\theta)$ & $\overline{1})$ & \\
\hline$\eta_{10 \mathrm{c}}$ & $\eta_{2 A}$ & $\overline{\eta_{2 B}}$ & $\eta_{2 c}$ & & \\
\hline$\left(\begin{array}{lll}\theta & 0 & 1\end{array}\right)$ & & & & & $\bar{I}$ \\
\hline$\frac{\eta_{10 D}}{(\theta \Theta \theta 1)}$ & $\eta_{2 A}$ & & $\begin{array}{ll}2 B \\
\theta 1)\end{array}$ & $\eta_{2 c}$ & $\eta_{20}$ \\
\hline 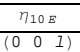 & $\eta_{2 A}$ & $\eta_{2 B}$ & & $\frac{c}{1}$ & $\eta_{2 L}$ \\
\hline$\eta_{10 F}$ & $\eta_{2 A}$ & & & $\eta_{20}$ & $\eta_{2 l}$ \\
\hline$\left(\begin{array}{lll}0 & 0 & 1\end{array}\right)$ & & & $\theta \bar{I}$ & & \\
\hline$\eta_{10 \mathrm{G}}$ & & & $\eta_{2 B}$ & $\eta_{2 c}$ & $\eta_{22}$ \\
\hline$\left(\begin{array}{lll}0 & 0 & 1\end{array}\right)$ & $(\theta$ & $\bar{I})$ & & & \\
\hline$\frac{\eta_{10 \mathrm{H}}}{(\theta \theta-1)}$ & $\eta_{2 A}$ & $\eta_{2 B}$ & $7_{2 c}$ & & \\
\hline $\begin{array}{lll}\left(\begin{array}{lll}0 & 1\end{array}\right) \\
\end{array}$ & & & & & \\
\hline
\end{tabular}

$\left(\eta_{10}, \eta_{3}\right)$

\begin{tabular}{|c|c|c|c|c|}
\hline$\frac{\eta_{10 \mathrm{~A}}}{\left(00 \theta^{\prime} \quad 1\right)}$ & \begin{tabular}{|c|}
$\eta_{3 A}$ \\
$(001)$
\end{tabular} & \begin{tabular}{|l|l|}
$7_{38}$ \\
\end{tabular} & $73 c$ & $\eta_{30}$ \\
\hline$\eta_{10 B}$ & \begin{tabular}{l|l}
$\eta_{3 A}$ & $\eta_{3 B}$
\end{tabular} & $\eta_{30}$ & & 730 \\
\hline$\left(\begin{array}{lll}(0 & 0 & 1\end{array}\right)$ & & (ब) & I) & \\
\hline$\eta_{10 c}$ & \begin{tabular}{l|l|l}
$\eta_{3 A}$ & $\eta_{3 B}$
\end{tabular} & $\mid \eta_{3 c}$ & & \\
\hline$\left(\begin{array}{lll}0 & 0 & 1\end{array}\right)$ & & & & OI) \\
\hline$\eta_{100}$ & \begin{tabular}{|l|l}
$\eta_{3 A}$ & $\eta_{3}$ \\
\end{tabular} & $3 B$ & $\eta_{3 c}$ & $\eta_{30}$ \\
\hline$\left(\begin{array}{lll}0 & 0 & 1\end{array}\right)$ & \begin{tabular}{|l|ll} 
& 100 \\
\end{tabular} & (21) & & \\
\hline $\begin{array}{l}\frac{\eta_{10 E}}{(001)} \\
01)\end{array}$ & \begin{tabular}{|l|l|}
$\eta_{3 A}$ & $\eta_{3 B}$ \\
\end{tabular} & $\eta_{3 \mathrm{C}}$ & & $\eta_{30}$ \\
\hline $\begin{array}{lll}0 & 0 & 1) \\
\end{array}$ & & & 1) & \\
\hline$\eta_{10 F}$ & \begin{tabular}{l|l}
$\eta_{3 A}$ & $\eta_{3}$ \\
\end{tabular} & & $\eta_{33}$ & 730 \\
\hline $\begin{array}{lll}\left(\begin{array}{lll}0 & 0 & 1\end{array}\right)\end{array}$ & \begin{tabular}{l|ll} 
& 100
\end{tabular} & (बI) & & \\
\hline$\eta_{106}$ & $\eta_{3 A}$ & $\eta_{3 B}$ & $\eta_{3}$ & $\eta_{30}$ \\
\hline $\left.\begin{array}{lll}(0 & 0 & 1\end{array}\right)$ & 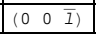 & & & \\
\hline \begin{tabular}{|c|}
$\eta_{10 \mathrm{H}}$ \\
$(001) \quad 1)$
\end{tabular} & \begin{tabular}{l|l|}
$\eta_{3 A}$ & $\eta_{3 B}$ \\
\end{tabular} & $n_{3 c}$ & & 30 \\
\hline & & & & (1) \\
\hline
\end{tabular}


$\left(\eta_{11}, \eta_{1}\right)$

\begin{tabular}{|c|c|c|c|c|c|c|c|}
\hline$\eta_{11 A}$ & \multicolumn{2}{|c|}{ 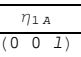 } & $\eta_{1 B}$ & $\eta_{1 c}$ & $\eta_{10}$ & $\eta_{1 E}$ & $\eta_{1 F}$ \\
\hline$\eta_{118}$ & $\eta_{1}$ & & $\eta_{1 B}$ & $\begin{array}{l}\eta_{1 c} \\
\text { (n) }\end{array}$ & $\eta_{10}$ & $\bar{\eta}_{1 E}$ & $\eta_{1 F}$ \\
\hline$\left(\begin{array}{llll}0 & 0\end{array}\right)$ & $\left(\begin{array}{ll}0 & 0\end{array}\right.$ & $\overline{1})$ & & & & & \\
\hline 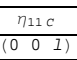 & \begin{tabular}{l|}
$\eta_{1 A}$ \\
\end{tabular} & & & $\eta_{1 c}$ & $\eta_{10}$ & $\eta_{1 E}$ & $\eta_{1 F}$ \\
\hline$\eta_{110}$ & $\eta_{1 A}$ & $\eta_{1}$ & & $\eta_{1 c}$ & $\eta_{10}$ & $\eta_{1 E}$ & $\eta_{1 F}$ \\
\hline$\left(\begin{array}{lll}0 & 0 & 1\end{array}\right)$ & & $(\theta)$ & $\bar{I})$ & & & & \\
\hline$\frac{\eta_{11 E}}{(\theta 01)}$ & $\eta_{1 \mathrm{~A}}$ & $\eta_{1 B}$ & $\frac{\eta_{1}}{100}$ & $\frac{c}{c}$ & $\eta_{10}$ & $\eta_{1 E}$ & $\eta_{1 F}$ \\
\hline$\eta_{11 F}$ & $\eta_{1 A}$ & $\eta_{18}$ & $\eta_{11}$ & & $\eta_{10}$ & $\eta_{1 E}$ & $\eta_{1 F}$ \\
\hline$\left(\begin{array}{lll}0 & 0 & 1\end{array}\right)$ & & & 100 & $\bar{I}$ & & & \\
\hline 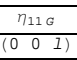 & $\eta_{1 A}$ & $\eta_{1 B}$ & $\eta_{1 c}$ & & & $\eta_{1 E}$ & $\eta_{1 F}$ \\
\hline$\eta_{11 H}$ & $\eta_{1 \mathrm{~A}}$ & $\eta_{1 B}$ & $\mid \begin{array}{ll}\eta_{11} \\
\end{array}$ & $\eta_{1}$ & & $\eta_{1 E}$ & $\eta_{1 F}$ \\
\hline$\left(\begin{array}{lll}0 & 0 & 1\end{array}\right)$ & & & & $(\theta)$ & (I) & & \\
\hline$\frac{\eta_{11 I}}{\left(\begin{array}{llll}0 & 0 & 1\end{array}\right)}$ & $\eta_{1 \mathrm{~A}}$ & $\eta_{1 B}$ & $\eta_{1 c}$ & $\eta_{10}$ & & E & $\eta_{1 F}$ \\
\hline$\eta_{111}$ & $\eta_{1 A}$ & $\eta_{1 B}$ & $\eta_{1 c}$ & $\eta_{10}$ & $\eta_{11}$ & & $\eta_{1 F}$ \\
\hline$\left(\begin{array}{lll}0 & 0 & 1\end{array}\right)$ & & & & & & $\bar{I})$ & \\
\hline$\frac{\eta_{11 K}}{(001)}$ & $\eta_{1 A}$ & $\eta_{1 B}$ & $\eta_{1 c}$ & $\eta_{10}$ & $\eta_{1 E}$ & & IF \\
\hline$\eta_{11 L}$ & $\eta_{1 A}$ & $\eta_{1 B}$ & $\eta_{1 c}$ & $\eta_{10}$ & $\eta_{1 E}$ & $n_{1}$ & \\
\hline$\left(\begin{array}{lll}0 & 0 & 1\end{array}\right)$ & & & & & & & $\bar{I})$ \\
\hline
\end{tabular}

$\left(\eta_{11}, \eta_{2}\right)$

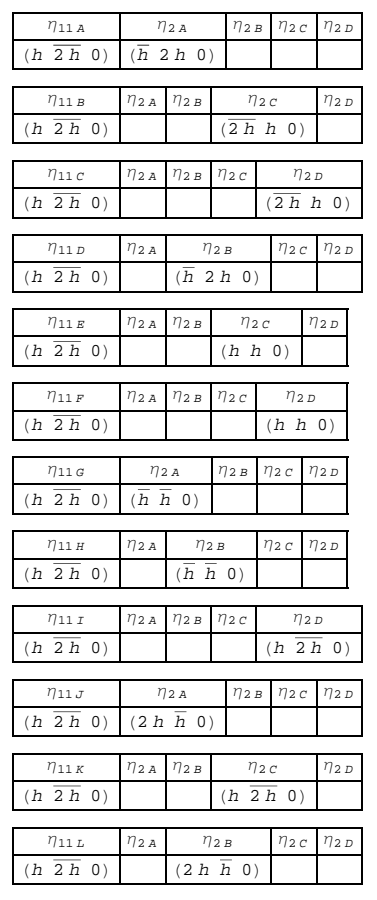


$\left(\eta_{11}, \eta_{4}\right)$

\begin{tabular}{|c|c|c|c|c|c|c|c|c|c|c|c|c|c|c|}
\hline & \multirow{2}{*}{\multicolumn{2}{|c|}{$\frac{\eta_{4 A}}{\left(\begin{array}{lll}\theta & 0 & 1\end{array}\right)}$}} & & \multirow{2}{*}{\multicolumn{2}{|c|}{\begin{tabular}{c|c}
$c$ & $\eta_{4}$ \\
\end{tabular}}} & \multirow[t]{2}{*}{$\eta_{4 E}$} & \multirow[t]{2}{*}{$74 \mathrm{~F}$} & \multirow[t]{2}{*}{$4 G$} & \multirow[t]{2}{*}{$74 \mathrm{H}$} & \multirow{2}{*}{74 . } & & \multirow{2}{*}{\multicolumn{2}{|c|}{\begin{tabular}{|l|l|}
$\eta_{4 k}$ & $\eta_{4}$ \\
& \\
\end{tabular}}} \\
\hline $\begin{array}{lll}0 & 11\end{array}$ & & & $(\theta)$ & I) & & & & & & & & & & \\
\hline$\eta_{11 \mathrm{e}}$ & $\frac{n_{2}}{2}$ & $4 A$ & $\eta_{4}$ & $4 B$ & & $n_{40}$ & $\eta_{4 E}$ & $\eta_{4 F}$ & $7_{46}$ & $7_{4 \mathrm{H}}$ & $\eta_{4 I}$ & $74 \mathrm{~J}$ & $\eta_{4 K}$ & \\
\hline 01 (1) & $(\theta)$ & $\begin{array}{l}\theta \bar{I}\rangle \\
\end{array}$ & $(\theta)$ & 1) & & & & & & & & & & \\
\hline$\eta_{11 c}$ & & $\eta_{48}$ & $n_{4}$ & & $\eta_{4}$ & & $\begin{array}{l}74 E \\
\end{array}$ & $\eta_{4 \mathrm{~F}}$ & $7_{46}$ & $\eta_{4 \mathrm{H}}$ & $7_{4 I}$ & $7_{4 \mathrm{~J}}$ & $\eta_{4 k}$ & \\
\hline $\begin{array}{lll}\theta & 1) \\
\end{array}$ & & & $\angle 00$ & 1) & 100 & $\bar{I}$ & & & & & & & & \\
\hline$\eta_{110}$ & $\eta_{4 A}$ & $\eta_{48}$ & $\eta_{4}$ & & $\eta_{4}$ & & $\eta_{4 E}$ & $\eta_{4 F}$ & $n_{46}$ & $\eta_{44}$ & $\eta_{41}$ & $\eta_{40}$ & $\eta_{4 k}$ & $\eta_{4}$ \\
\hline $\begin{array}{llll}0 & 0 & 1) \\
\end{array}$ & & & $(\theta 0)$ & I) & 100 & 0 1) & & & & & & & & \\
\hline$\eta_{11 \mathrm{E}}$ & $\eta_{4 \mathrm{~A}}$ & $\eta_{4 B}$ & $\eta_{4 c}$ & $\eta_{40}$ & $\eta_{4}$ & & $\eta_{4}$ & & $\eta_{46}$ & $\eta_{4 \mathrm{H}}$ & $\eta_{4 I}$ & $74 \mathrm{~J}$ & $74 \mathrm{~K}$ & 74 \\
\hline $\begin{array}{lll}0 & 0 & 1\end{array}$ & & & & & 100 & (1) & 100 & eI) & & & & & & \\
\hline$\eta_{11 F}$ & $\eta_{4 A}$ & $7_{4 B}$ & $7_{4 c}$ & $7_{40}$ & $\eta_{4}$ & & $\eta_{4}$ & & $\eta_{4 G}$ & $7_{44}$ & $\eta_{4 I}$ & $74 \mathrm{~J}$ & $n_{4 k}$ & $n_{4}$ \\
\hline $\begin{array}{llll}0 & 11\end{array}$ & & & & & 100 & (0) & $\angle \theta 0$ & & & & & & & \\
\hline$\eta_{11 G}$ & $\eta_{4 \mathrm{~A}}$ & $7_{4 B}$ & $\eta_{4 c}$ & $n_{4 D}$ & \begin{tabular}{|l|}
$\eta_{4 E}$ \\
\end{tabular} & $74 \mathrm{~F}$ & $\eta_{4}$ & & $\eta_{4}$ & & $\eta_{4 I}$ & $74 \mathrm{~J}$ & $\eta_{4 K}$ & $n_{4}$ \\
\hline $\begin{array}{lll}0 & 0 & 1\end{array}$ & & & & & & & & $01)$ & 100 & I) & & & & \\
\hline$\eta_{11 H}$ & $\eta_{4 A}$ & $7_{4 B}$ & $74 c$ & $7_{4 D}$ & \begin{tabular}{|l|}
$\eta_{4 E}$ \\
\end{tabular} & $\eta_{4} \mathrm{~F}$ & $\eta_{4}$ & & $\bar{\eta}_{4}$ & & $74 I$ & $77_{4 \mathrm{~J}}$ & $\eta_{4 k}$ & 74 \\
\hline 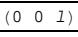 & & & & & & & & (बI) & $10 \theta$ & & & & & \\
\hline$\eta_{11 I}$ & $\eta_{4 \mathrm{~A}}$ & $\eta_{48}$ & $7_{44}$ & $\eta_{40}$ & \begin{tabular}{|l|}
$7_{4 E}$ \\
\end{tabular} & $\eta_{4 \mathrm{~F}}$ & $\eta_{46}$ & $\eta_{44}$ & $\eta_{4}$ & & $\bar{\eta}$ & & $7_{4 k}$ & \\
\hline $\begin{array}{llll}0 & 0 & 1)\end{array}$ & & & & & & & & & 10 & 91) & & $\begin{array}{l}0 \overline{1}) \\
\end{array}$ & & \\
\hline$\eta_{11 .}$ & $n_{4 A}$ & $\eta_{48}$ & 740 & $n_{40}$ & \begin{tabular}{|l|l|} 
\\
\end{tabular} & $\eta_{44}$ & $\eta_{46}$ & $\eta_{4 \mathrm{H}}$ & $n_{4}$ & & $\eta$ & & $\eta_{4 K}$ & \\
\hline $\begin{array}{lll}0 & 0 & 1)\end{array}$ & & & & & & & & & & (D) & & $01)$ & & \\
\hline$\eta_{11 k}$ & $\eta_{4 A}$ & $\eta_{48}$ & 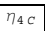 & $\eta_{40}$ & $\eta_{4 E}$ & $\eta_{4 F}$ & $\eta_{46}$ & $\eta_{4 H}$ & 741 & $74 \mathrm{~J}$ & & & $\eta_{z}$ & \\
\hline$\left(\begin{array}{lll}0 & 0 & 1\end{array}\right)$ & & & & & & & & & & & & $\begin{array}{lll}0 & 1\end{array}$ & & $\begin{array}{ll}(\bar{I}) \\
\end{array}$ \\
\hline$\eta_{11}$ & $\eta_{4 A}$ & $\eta_{48}$ & $\eta_{40}$ & $\eta_{40}$ & \begin{tabular}{|l|l|}
$74 E$ \\
\end{tabular} & $7_{44} \mathrm{~F}$ & $\eta_{4 G}$ & $\eta_{4 H}$ & $\eta_{4 I}$ & $\eta_{43} \mathrm{~J}$ & $\eta$ & & $\eta_{4}$ & \\
\hline$\theta$ & & & & & & & & & & & & $\theta \bar{I})$ & & \\
\hline
\end{tabular}


$\left(\eta_{11}, \eta_{8}\right)$

\begin{tabular}{|c|c|c|c|c|c|c|c|c|c|c|c|c|c|c|c|c|c|c|c|c|c|c|c|c|c|c|c|}
\hline & & $8 A$ & 788 & $78 \mathrm{c}$ & 780 & $\eta_{8 E}$ & $\eta_{85}$ & 78 & $G$ & & & $78 \mathrm{~J}$ & $\bar{\eta}_{8 K}$ & 784 & $\eta_{81}$ & & $\eta_{8 \mathrm{~N}}$ & 780 & $\eta_{8 P}$ & 780 & \begin{tabular}{ll|}
$7_{8 R}$ \\
\end{tabular} & $\overline{78 S}_{85}$ & $\eta_{8 T}$ & $\eta_{80}$ & $7_{8 \mathrm{v}}$ & $7_{8 \mathrm{w}}$ & $78 x$ \\
\hline$\overline{2 h} \theta)$ & & $h \theta)$ & & & & & & & & & & & & & & 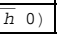 & & & & & & & & & & & \\
\hline 118 & $78 \mathrm{~A}$ & & & $7_{8 \mathrm{C}}$ & \begin{tabular}{c|c}
$c$ & 780 \\
\end{tabular} & $7_{8 E}$ & $\overline{785}$ & $\eta_{8}$ & $\eta_{8}$ & H & & $\eta_{8 \mathrm{~J}}$ & $\eta_{8 K}$ & $n_{81}$ & $78 \mathrm{M}$ & $\eta_{81}$ & $8 \mathrm{~N}$ & 780 & $\eta_{8 P}$ & 780 & \begin{tabular}{|l|r}
$n_{8 R}$ & 7
\end{tabular} & $\begin{array}{lll}7_{88} & 7\end{array}$ & \begin{tabular}{|l|l|}
$\eta_{8}$ & \\
\end{tabular} & 784 & $78 \mathrm{~V}$ & $78 \mathrm{~W}$ & $78 x$ \\
\hline$\overline{2 h} \theta$ & & $\begin{array}{ll}(\bar{h} 2 \\
\end{array}$ & $h \theta)$ & & & & & & & & & & & & & $\langle h \overline{2 h}$ & 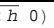 & & & & & & & & & & \\
\hline & $n_{8 A}$ & $\eta_{88}$ & $\eta_{8}$ & $8 c$ & $\eta_{80}$ & $\eta_{8 E}$ & $\eta_{8 f}$ & $7_{8}$ & $\eta_{8}$ & ${ }_{H}^{H}$ & 8 & $78 \mathrm{~J}$ & $\eta_{8 \mathrm{~K}}$ & $\eta_{8 L}$ & $\eta_{8 \mathrm{M}}$ & $7_{8 \mathrm{~N}}$ & $\eta_{80}$ & $\eta_{8}$ & & $\eta_{80}$ & $\eta_{8 R}$ & $\eta_{8 s}$ & $\eta_{8 T T}$ & 780 & $\eta_{8 V}$ & $\eta_{8 w}$ & $78 x$ \\
\hline$\overline{250}$ & & & $\begin{array}{ll}4 h 2 \\
\end{array}$ & $2 h 0)$ & & & & & & & & & & & & & & $\begin{array}{ll}l n & 2 \\
\end{array}$ & (h⿱一𫝀) & & & & & & & & \\
\hline$\eta_{110}$ & $\eta_{8 \mathrm{~A}}$ & $n_{8 B}$ & $\eta_{8 \mathrm{C}}$ & & 80 & $\eta_{8 E}$ & $\eta_{8 \mathrm{~F}}$ & $\eta_{8}$ & \begin{tabular}{l|l}
6 & $\eta_{8}$
\end{tabular} & $H$ & & $\eta_{8 \mathrm{~J}}$ & $\eta_{8 K}$ & 784 & \begin{tabular}{|l|l}
$78 \mathrm{M}$ & $r$
\end{tabular} & \begin{tabular}{|l|}
$78 \mathrm{~N}$ \\
\end{tabular} & & 80 & $\eta_{8 p}$ & $7_{80}$ & $\eta_{8 R}$ & $\eta_{8 s}$ & $\eta_{8 T}$ & $78 u$ & \begin{tabular}{l|l}
$\eta_{8 \mathrm{~V}}$ & $\eta$
\end{tabular} & $78 \mathrm{w}$ & $78 x$ \\
\hline$\hbar \overline{2 h} \theta)$ & & & & $(\bar{h} 2$ & $2 h 0)$ & & & & & & & & & & & & & 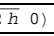 & & & & & & & & & \\
\hline$\eta_{11 E}$ & $7_{8 A}$ & $n_{88}$ & $n_{8 c}$ & 780 & & $8 E$ & $77_{8}$ & $7_{8}$ & \begin{tabular}{l|l}
6 & $\eta_{8}$ \\
\end{tabular} & ${ }_{H}$ & & $\begin{array}{ll}\eta_{8} & 1\end{array}$ & $\begin{array}{ll}n_{8 K} & 7\end{array}$ & $\eta_{8 L}$ & \begin{tabular}{l|l}
$n_{8} M$ & $r$
\end{tabular} & \begin{tabular}{|l|}
$78 \mathrm{~N}$ \\
\end{tabular} & 780 & $\eta_{8 P}$ & 780 & 88. & 85 & $78 \mathrm{~T}$ & 880 & $88 \mathrm{~V}$ & $\eta_{8 W}$ & & \\
\hline 250 & & & & & $(\bar{h} 2$ & $(n \theta)$ & & & & & & & & & & & & & & & & & & & $(h 2 h$ & 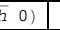 & \\
\hline$\eta_{11 F}$ & $78 \mathrm{~A}$ & 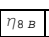 & \begin{tabular}{ll|}
$\eta_{8 c}$ \\
\end{tabular} & 780 & $\begin{array}{l}\eta_{8 E} \\
\end{array}$ & $\eta_{8}$ & & $n_{8}$ & \begin{tabular}{l|l}
6 & $\eta_{81}$
\end{tabular} & ${ }^{H}$ & & $7_{80}$ & $\begin{array}{l}7_{8 K} \\
\end{array}$ & & $8 M$ & $n_{8 \mathrm{~N}}$ & 80 & \begin{tabular}{l|l|l|l|l|}
$8 P$ & \\
\end{tabular} & $n_{80}$ & & & & 884 & $78 \mathrm{~V}$ & $78 \mathrm{w}$ & $\eta_{8 x}$ & \\
\hline 250 & & & & & & $(\overline{6} 21$ & $h 0$ & & & & & & & & & & & & & & & & & & & $h \overline{2 h}$ & (0) \\
\hline$\eta_{11}$ & $\overline{\eta_{8 A}}$ & $\eta_{8 B}$ & $\bar{n}_{8 \mathrm{C}}$ & $\eta_{8 D}$ & \begin{tabular}{|l|l|}
$n_{8 E}$ \\
\end{tabular} & $78 \mathrm{~F}$ & & & $\eta_{8}$ & $H$ & & $78 \mathrm{~J}$ & $\eta_{8 K}$ & 18 & $78 \mathrm{M}$ & $\eta_{8 \mathrm{~N}}$ & $\bar{n}_{80}$ & $78 \mathrm{P}$ & $n_{80}$ & $\eta_{8 R}$ & 785 & $8 T$ & 780 & $\eta_{8 \mathrm{v}}$ & & $\eta_{88}$ & $\eta_{8 x}$ \\
\hline $7 \overline{2 h \theta)}$ & & & & & & & $4 \bar{h} 2$ & $2 h \theta$ & & & & & & & & & & & & & & & & $(h \overline{2 h}$ & $\begin{array}{ll}50) \\
\end{array}$ & & \\
\hline & $\bar{n}_{8 A}$ & $n_{8 B}$ & $\eta_{8 \mathrm{c}}$ & $\eta_{80}$ & \begin{tabular}{|l|l|}
$78 E$ \\
\end{tabular} & $\bar{n}_{8 F}$ & $\eta_{86}$ & & $78 \mathrm{H}$ & $\bar{n}_{8}$ & & \begin{tabular}{l|l}
$78 \mathrm{~J}$ & 7
\end{tabular} & $78 K$ & $\eta_{8 L}$ & $\overline{\eta_{8} M}$ & $\eta_{8 \mathrm{~N}}$ & $\bar{r}_{80}$ & \begin{tabular}{l|l}
188 &
\end{tabular} & $\bar{n}_{80} 0$ & \begin{tabular}{ll|}
$8 R$ \\
\end{tabular} & $78 s$ & $78 \mathrm{~T}$ & $\eta_{8 u}$ & & \begin{tabular}{l|l}
$\eta_{8 \mathrm{~V}}$ & $\mathrm{~F}^{\prime}$
\end{tabular} & $\eta_{8} w$ & $\overline{88 x}$ \\
\hline$h \overline{2 h} \theta)$ & & & & & & & & & $2 \mathrm{hO}$ & & & & & & & & & & & & & & $h \overline{2 h}$ & i0) & & & \\
\hline$\eta_{11}$ & $78 \mathrm{AA}$ & 788 & $78 \mathrm{c}$ & 780 & $78 E$ & $\overline{n_{8 F}}$ & 786 & $\eta_{8 \mathrm{H}}$ & & 781 & & $\begin{array}{ll}8_{8} & 17 \\
\end{array}$ & $78 \mathrm{~K}$ & $n_{84}$ & $7_{8 M}$ & \begin{tabular}{|l|l|}
$78 \mathrm{~N}$ \\
\end{tabular} & 780 & \begin{tabular}{ll|}
${ }_{8} P$ \\
\end{tabular} & $\eta_{80}$ & \begin{tabular}{l|l}
$7_{8 R}$ & \\
\end{tabular} & ${ }_{88}$ & $78 \mathrm{~T}$ & & \begin{tabular}{|l|l}
$n_{80}$ \\
\end{tabular} & \begin{tabular}{|l|r}
$\eta_{8}$ & $r$ \\
\end{tabular} & $\begin{array}{llll}n_{8} & 7\end{array}$ & $78 x$ \\
\hline$\overline{2 h 0}$ & & & & & & & & & & $2 h \theta$ & & & & & & & & & & & & & (n) & & & & \\
\hline$\eta_{11}$ & 4. & \begin{tabular}{|l|}
788 \\
\end{tabular} & \begin{tabular}{ll|}
$\eta_{80}$ \\
\end{tabular} & 780 & \begin{tabular}{ll|}
$78 E$ \\
\end{tabular} & \begin{tabular}{|l|l}
$7_{88}$ \\
\end{tabular} & 786 & $\eta_{8 \mathrm{H}}$ & $78 I$ & & $78 \mathrm{~J}$ & & $78 \mathrm{~K}$ & $\eta_{8 L}$ & $\overline{7}_{8 \mathrm{M}}$ & $n_{8 N}$ & $7_{80}$ & $\begin{array}{lll}788 & \\
\end{array}$ & 780 & $78 R$ & $n_{85}$ & & $\eta_{8 T} T$ & 780 & $\eta_{8 \mathrm{~V}}$ & \begin{tabular}{|l|l}
$78 w$ & 7 \\
\end{tabular} & $78 x$ \\
\hline 250 & & & & & & & & & & $\langle\overline{2}$ & $2 h$ & ब) & & & & & & & & & $\langle h \overline{2 h}$ & $\begin{array}{ll}50) \\
\end{array}$ & & & & & \\
\hline & $78 \mathrm{~A}$ & $77_{88}$ & $\eta_{8 \mathrm{c}}$ & 780 & \begin{tabular}{|l|l|}
$78 \mathrm{E}$ & \\
\end{tabular} & \begin{tabular}{|l|l}
788 &
\end{tabular} & 786 & $\overline{78 \mathrm{H}}$ & $78 I$ & $78 \mathrm{~J}$ & & & & \begin{tabular}{ll|}
81 \\
\end{tabular} & \begin{tabular}{l|l}
$78 \mathrm{M}$ \\
\end{tabular} & \begin{tabular}{l|l}
$78 \mathrm{~N}$ & $\mathrm{P}$
\end{tabular} & 780 & $\eta_{8 p}$ & $\eta_{8}$ & & \begin{tabular}{ll|}
$78 R$ \\
\end{tabular} & \begin{tabular}{|l|l}
$\eta_{85}$ &
\end{tabular} & \begin{tabular}{|l|l}
$\eta_{8}$ & 7 \\
\end{tabular} & \begin{tabular}{|l|l|}
$n_{8}$ \\
$\mid$
\end{tabular} & \begin{tabular}{l|l}
$\eta_{8} \mathrm{~V}$ \\
\end{tabular} & \begin{tabular}{|l|l}
$n^{\prime}$ &
\end{tabular} & $78 x$ \\
\hline$\overline{2 h} \theta$ & & & & & & & & & & & & $2 h$ & (0) & & & & & & & 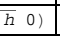 & & & & & & & \\
\hline$\eta_{11 L}$ & $78 \mathrm{~A}$ & $\eta_{88}$ & $n_{88}$ & 780 & $78 E$ & \begin{tabular}{ll|}
$78 F$ \\
\end{tabular} & 786 & $\overline{78 \mathrm{H}}$ & $78 I$ & $78 \mathrm{~J}$ & $\eta_{8}$ & K & $78 \mathrm{~L}$ & & \begin{tabular}{|l|l}
$78 M$ \\
\end{tabular} & \begin{tabular}{|l|l|}
$78 \mathrm{~N}$ \\
\end{tabular} & 780 & $78 P$ & 780 & 78 & \begin{tabular}{|l|l|}
$8 R$ \\
\end{tabular} & $\eta_{8 s}$ & \begin{tabular}{|l|l}
$7_{81}$ &
\end{tabular} & \begin{tabular}{|l|l}
780 \\
\end{tabular} & \begin{tabular}{l|l}
$n_{88 v}$ & $n$
\end{tabular} & $78 \mathrm{~W}$ & $78 x$ \\
\hline$\overline{2 h} \theta)$ & & & & & & & & & & & & & $2 h$ & & & & & & & & $\bar{n} \bar{n}$ ๑) & & & & & & \\
\hline
\end{tabular}

$\left(\eta_{11}, \eta_{9}\right)$

\begin{tabular}{|c|c|c|c|c|c|c|c|}
\hline 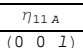 & \multicolumn{2}{|c|}{ 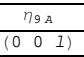 } & \begin{tabular}{l|l}
798 & \\
9
\end{tabular} & $\eta_{9 c}$ & 790 & $79 \mathrm{E}$ & $\eta_{9 \mathrm{~F}}$ \\
\hline$\eta_{11 \mathrm{~B}}$ & \multicolumn{2}{|c|}{$\eta_{9 A}$} & $\eta_{9 B}$ & $\eta_{9 c}$ & $n_{90}$ & $\overline{\eta_{9 F}}$ & $n_{\eta_{9 F}}$ \\
\hline$\left(\begin{array}{lll}0 & 0 & 1\end{array}\right)$ & \multicolumn{2}{|c|}{$\frac{19 \mathrm{~A}}{(\theta \theta I)}$} & & & & & \\
\hline 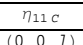 & \multicolumn{3}{|c|}{\begin{tabular}{|c|c|}
$A$ & $\eta_{9 B}$ \\
& $\left(\begin{array}{lll}0 & 0 & 1\end{array}\right)$ \\
\end{tabular}} & \begin{tabular}{ll|}
$7_{9 c}$ \\
\end{tabular} & $\eta_{90}$ & $\eta_{9 E}$ & $\eta_{9 \mathrm{~F}}$ \\
\hline$\eta_{110}$ & $\eta_{9 A}$ & \multirow{2}{*}{\multicolumn{3}{|c|}{\begin{tabular}{c|c|cc}
4 & $\eta_{9 B}$ & $\eta_{90}$ \\
$\theta 0$ & 0
\end{tabular}}} & $\eta_{90}$ & $\bar{\eta}_{9 E}$ & $\eta_{9 F}$ \\
\hline$\left(\begin{array}{lll}0 & 0 & 1\end{array}\right)$ & & & & $\left.\mid \begin{array}{lll}(0 & 0 & 1\end{array}\right)$ & & & \\
\hline$\eta_{11 E}$ & $\eta_{9 A}$ & \multicolumn{3}{|c|}{\begin{tabular}{|c|c|}
$\eta_{98}$ & $\eta_{9 c}$ \\
\end{tabular}} & 790 & $\eta_{9 E}$ & $\eta_{9 \mathrm{~F}}$ \\
\hline$\eta_{11 F}$ & \multicolumn{4}{|c|}{\begin{tabular}{l|l|l|}
$7_{9 A}$ & $\eta_{9 B}$ & $\eta_{9 C}$ \\
\end{tabular}} & & $\eta_{9 E}$ & $\eta_{9 F}$ \\
\hline$\left(\begin{array}{lll}0 & 0 & 1\end{array}\right)$ & & \multicolumn{4}{|c|}{\begin{tabular}{c|c}
$\eta_{98}$ & $\eta_{9 c}$ \\
& $\left(\begin{array}{lll}\theta & 0 & I\end{array}\right)$
\end{tabular}} & & \\
\hline $\begin{array}{l}\frac{\eta_{116}}{(001)} \\
(001)\end{array}$ & \multicolumn{2}{|c|}{\begin{tabular}{|l|l|}
$A$ & $7_{98}$ \\
\end{tabular}} & $\eta_{9 c}$ & \multicolumn{2}{|c|}{\begin{tabular}{|c|}
$\eta_{9 D}$ \\
$(\theta 001)$
\end{tabular}} & $\eta_{9 E}$ & $\eta_{9 F}$ \\
\hline$\eta_{11 \mathrm{H}}$ & \multirow{2}{*}{\multicolumn{2}{|c|}{$\begin{array}{ll}\eta_{98} \\
\eta_{9}\end{array}$}} & $n_{9 c}$ & \multirow{2}{*}{\multicolumn{2}{|c|}{\begin{tabular}{|l|}
$\eta_{9 D}$ \\
$\theta \theta I$
\end{tabular}}} & $\eta_{9 E}$ & $\eta_{9 F}$ \\
\hline$\left(\begin{array}{lll}0 & 0 & 1\end{array}\right)$ & & & & & & & \\
\hline$\eta_{11 I}$ & $\eta_{9 A}$ & $7_{9 B}$ & $\eta_{9 c}$ & 790 & & & $7_{9 \mathrm{~F}}$ \\
\hline 01$)$ & & & & & & 1) & \\
\hline$\eta_{11} \mathrm{~J}$ & $\eta_{9 A}$ & \begin{tabular}{|l|}
$79 B$ \\
7
\end{tabular} & $\eta_{9 c}$ & \begin{tabular}{|l|}
790 \\
\end{tabular} & $\eta_{9}$ & & $\eta_{9 F}$ \\
\hline$\left(\begin{array}{lll}\theta & 0 & 1\end{array}\right)$ & & & & & & I) & \\
\hline$\eta_{11 K}$ & $\eta_{9 A}$ & $\eta_{98}$ & $\eta_{9 c}$ & $\eta_{90}$ & $\eta_{9 \mathrm{E}}$ & & $9 F$ \\
\hline$\left(\begin{array}{lll}0 & 0 & 1\end{array}\right)$ & & & & & & & \\
\hline$\eta_{11 L}$ & $\eta_{9 A}$ & $\eta_{9 B}$ & $\eta_{9 c}$ & \begin{tabular}{|l|l|}
$7_{90}$ \\
\end{tabular} & $\eta_{9 \mathrm{E}}$ & $\eta_{s}$ & \\
\hline$\left(\begin{array}{lll}\theta & 0 & 1\end{array}\right)$ & & & & & & & (बI) \\
\hline
\end{tabular}




\begin{tabular}{|c|c|c|c|c|c|c|}
\hline$\eta_{12 \mathrm{~A}}$ & $\eta_{9 \mathrm{~A}}$ & $\eta_{9 B}$ & $\eta_{9 c}$ & $\eta_{90}$ & \begin{tabular}{|l|}
$n_{9 E}$ \\
\end{tabular} & $\eta_{9 f}$ \\
\hline 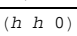 & & & $\langle\bar{h} 2 h \quad \theta\rangle$ & $\langle(h \overline{2 h} \theta\rangle$ & & \\
\hline$\eta_{12 B}$ & $n_{9 \mathrm{~A}}$ & $\eta_{9 B}$ & $\eta_{9 c}$ & $\eta_{90}$ & \begin{tabular}{|l|}
$n_{9 E}$ \\
\end{tabular} & $\eta_{96}$ \\
\hline$h h \theta)$ & & & $(h \overline{2 h} \theta\rangle$ & $\langle\bar{h} 2 h \quad 9\rangle$ & & \\
\hline$\eta_{12 c}$ & & $79 \mathrm{~A}$ & $\eta_{9 B}$ & \begin{tabular}{|l|l|}
$\eta_{9 c}$ & $\eta_{90}$ \\
\end{tabular} & $\eta_{9 E}$ & $\eta_{9 F}$ \\
\hline 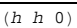 & $\langle h \overline{2}$ & $\overline{2 h}$ 0) & $\langle\bar{h} 2 h \theta$ & & & \\
\hline$\eta_{120}$ & & $79 \mathrm{~A}$ & $\eta_{9 B}$ & \begin{tabular}{l|l}
$\eta_{9 c}$ & $\eta_{90}$
\end{tabular} & $\eta_{g_{E}}$ & $\eta_{99}$ \\
\hline 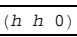 & $(\bar{h} 2$ & $2 h \theta)$ & $(h \overline{\bar{h}} \mathrm{\theta}$ & & & \\
\hline$\eta_{12 E}$ & $\eta_{9 \mathrm{AA}}$ & $\eta_{98}$ & \begin{tabular}{l|l}
$\eta_{9 c}$ & $\eta_{9 D}$
\end{tabular} & $\eta_{9 E}$ & $\eta_{996}$ & \\
\hline$h h \theta$ & & & & $\left(\begin{array}{llll}h & 2 h & \theta\end{array}\right)$ & $(h 2 h$ & $\bar{h}$ 0) \\
\hline$\eta_{12 F}$ & $n_{9 \mathrm{~A}}$ & $7_{9 B}$ & \begin{tabular}{l|l}
$\eta_{9 c}$ & $\eta_{90}$ \\
\end{tabular} & $\eta_{9 \mathrm{E}}$ & $\eta_{91}$ & \\
\hline 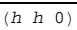 & & & & $\langle(h \overline{2 h} 0)$ & & $h$ ब \\
\hline$\eta_{12 \mathrm{G}}$ & $\eta_{9 A}$ & $7_{9 B}$ & $\eta_{9 c}$ & $\eta_{90}$ & $\eta_{9 E}$ & $\eta_{9 F}$ \\
\hline 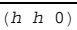 & & & $\begin{array}{ll}(h \overline{2 h} \theta & 0\end{array}$ & $\left(\begin{array}{llll}(h & 2 h & \theta\end{array}\right.$ & & \\
\hline$\eta_{12 \mathrm{H}}$ & \begin{tabular}{|l|}
$7_{9 \mathrm{~A}}$ \\
\end{tabular} & $\eta_{9 B}$ & $\eta_{9 c}$ & $\eta_{90}$ & \begin{tabular}{|l|}
$\eta_{9 E}$ \\
\end{tabular} & $\eta_{9 F}$ \\
\hline$(h \quad h \quad \theta)$ & & & $(\bar{h} 2 h \theta)$ & \begin{tabular}{|ll}
$(h \overline{2 h} \theta$ & 0
\end{tabular} & & \\
\hline$\eta_{12 I}$ & & $79 \mathrm{~A}$ & $\eta_{9 B}$ & \begin{tabular}{|l|l|l}
$\eta_{90}$ & $\eta_{90}$ \\
\end{tabular} & $\eta_{9 E}$ & $\eta_{9 F}$ \\
\hline$(h h \theta)$ & (h) 2 & $2 h \theta)$ & $\langle h \overline{2 h \theta}$ & & & \\
\hline$\eta_{12 \mathrm{~J}}$ & $\eta$ & $79 \mathrm{~A}$ & $\eta_{9 B}$ & \begin{tabular}{|l|l|}
$\eta_{9 c}$ & $\eta_{9 D}$ \\
\end{tabular} & $\eta_{9 E}$ & $7_{9 \mathrm{~F}}$ \\
\hline$\left(\begin{array}{lll}h & h & 0\end{array}\right)$ & $\langle(h \overline{2}$ & $2 \overline{2 h} 0$ & $\langle\bar{h} 2 \mathrm{h \theta}$ & & & \\
\hline$\eta_{12 k}$ & \begin{tabular}{|l|}
$n_{9 A}$ \\
\end{tabular} & 7 & \begin{tabular}{l|l|}
$\eta_{9 c}$ & $\eta_{90}$ \\
\end{tabular} & $\eta_{9 E}$ & $\eta_{9 f}$ & \\
\hline$h h \Theta$ & & & & $\left(\begin{array}{l}(h \overline{2 h} \\
\end{array}\right)$ & $(\bar{h} 2 h$ & $h$ o) \\
\hline $\bar{\eta}_{12 L}$ & \begin{tabular}{|l|}
$\eta_{9 A}$ \\
\end{tabular} & $7_{98}$ & \begin{tabular}{l|l}
$\eta_{9 c}$ & $\eta_{90}$
\end{tabular} & $\eta_{\eta_{9 E}}$ & $\eta_{99}$ & F \\
\hline$h \theta$ & & & & $(\bar{h} 2 h \quad 0)$ & $(h \overline{2 h}$ & \\
\hline
\end{tabular}


(iii) $:\left(\eta_{i}, \eta_{j}, \eta_{k} \ldots \ldots.\right)$

$\{h h 0\}$

First table should be read vertically the others horisontally

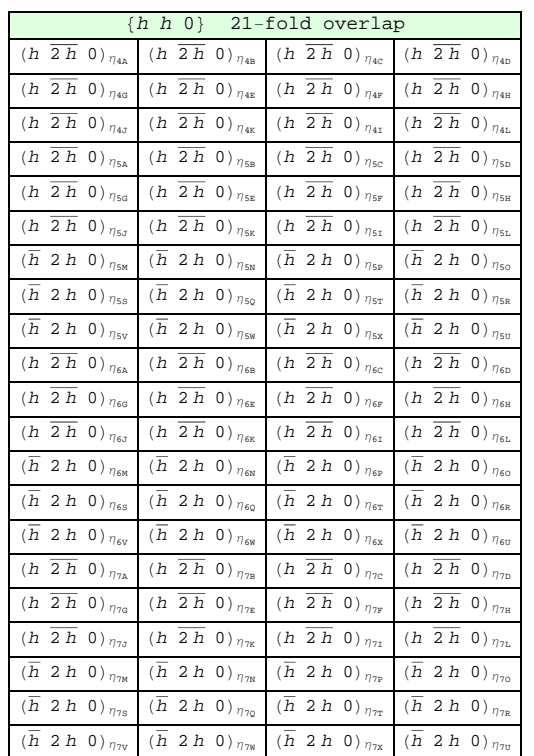

\begin{tabular}{|c|c|c|c|c|c|}
\hline \multicolumn{6}{|c|}{$\begin{array}{lll}h & h & 0\end{array}$}$\quad 6$-fold overlap \\
\hline$(h 2 h \theta)_{\eta_{9 A}}$ & $\left(\begin{array}{llll}(\hbar & 2 & h & 0\end{array}\right)_{\eta_{98}}$ & $\left(\begin{array}{lll}h & h & 0\end{array}\right)_{n_{1}}$ & $\left(\begin{array}{llll}h & h & \ddots\end{array}\right)_{\eta_{12 J}}$ & $(\hbar \hbar \theta)_{\eta_{120}}$ & $(\hbar \hbar \odot)_{\eta_{121}}$ \\
\hline$(h \overline{2 h} \Theta)_{\eta 9 c}$ & $\left(\begin{array}{llll}h & 2 & h & 0\end{array}\right)_{790}$ & $\left(\begin{array}{lll}(h \quad h \quad & 0\end{array}\right)_{\eta_{13}}$ & $\left(\begin{array}{lll}h & h & 0\end{array}\right)_{\eta_{126}}$ & $(\hbar \hbar \odot)_{\eta_{12 A}}$ & $(\bar{h} \bar{\dagger} \theta)_{\eta_{12 H}}$ \\
\hline$(h \overline{2 h} \theta)_{\eta_{9 E}}$ & $\left(\begin{array}{llll}h & 2 h & \ddots\end{array}\right)_{\eta_{9 F}}$ & $\left(\begin{array}{llll}h & h & 0\end{array}\right)_{\eta_{12}}$ & $(h \quad h \odot)_{\eta_{12 k}}$ & $(\bar{h} \bar{h} \odot)_{\eta_{12 \mathrm{E}}}$ & $(\hbar \hbar \Theta)_{\eta_{12 L}}$ \\
\hline
\end{tabular}

\begin{tabular}{|c|c|c|c|}
\hline \multicolumn{4}{|c|}{$\left.\begin{array}{llll}h & h & 0\end{array}\right\}$ 4-fold overlap } \\
\hline$\left(\begin{array}{llll}h & h & 0\end{array}\right)_{\eta_{2 A}}$ & $(h \quad 2 h \quad 0)_{\eta_{86}}$ & $\left(\begin{array}{llll}h & 2 & \ddots & \ddots\end{array}\right)_{\eta_{8 \mathrm{~V}}}$ & $\left(\begin{array}{llll}h & 2 & h & 0\end{array}\right)_{\eta_{116}}$ \\
\hline$\left(\begin{array}{llll}h & h & 0\end{array}\right)_{\eta_{2 B}}$ & $(h \overline{2 h} \odot)_{\eta_{\text {BH }}}$ & $\left(\begin{array}{llll}h & 2 & h & \ddots\end{array}\right)_{\eta_{8 \mathrm{~B}}}$ & $\left(\begin{array}{llll}\hbar & 2 h & \theta\end{array}\right)_{\eta_{11 H}}$ \\
\hline$(h \quad h \quad \odot)_{\eta_{2 c}}$ & $(h \overline{2 h} \odot)_{\text {new }}$ & $\left(\begin{array}{llll}\hbar & 2 & h & 0\end{array}\right)_{\eta_{\mathrm{BE}}}$ & $(h \overline{2 h} \theta)_{\eta_{11 E}}$ \\
\hline$\left(\begin{array}{llll}h & h & 0\end{array}\right)_{\eta_{20}}$ & $(h 2 h 0)_{\eta_{B x}}$ & $\left(\begin{array}{llll}h & 2 & h & 0\end{array}\right)_{\eta_{B F}}$ & $(h 2 h 0)_{\eta_{11 F}}$ \\
\hline$(h \overline{2 h} \theta)_{\eta_{2 A}}$ & $(h \overline{2 h} 0)_{\eta_{B A}}$ & $\left(\begin{array}{llll}(h & 2 & h & \ddots\end{array}\right)_{\eta_{8 \mathrm{~B}}}$ & $\left(\begin{array}{llll}h & 2 & h & \theta\end{array}\right)_{\eta_{11 \mathrm{~A}}}$ \\
\hline$(h \overline{2 h} \theta)_{\eta_{2 B}}$ & $(h \overline{2 h} 0)_{\eta_{80}}$ & $\left(\begin{array}{llll}h & 2 & h & \ddots\end{array}\right)_{\eta_{80}}$ & $\left(\begin{array}{llll}h & 2 & h & 0\end{array}\right)_{\eta_{110}}$ \\
\hline$(h 2 h \theta)_{\eta_{2 c}}$ & $(h 2 h 0)_{8 Q}$ & $(\hbar 2 h 0)_{\text {ngk }}$ & $(h \overline{2 h} \theta)_{\eta_{11 k}}$ \\
\hline$(h \overline{2 h} \theta)_{\eta_{20}}$ & $(h \overline{2 h} \odot)_{\eta_{\mathrm{BT}}}$ & $\left(\begin{array}{llll}h & 2 & h & 0\end{array}\right)_{\eta_{B I}}$ & $(h \overline{2 h} \theta)_{\eta_{11 I}}$ \\
\hline$(\overline{2 h} h \theta)_{\eta_{2 A}}$ & $(h \overline{2 h} 0)_{\eta_{83}}$ & $\left.\begin{array}{lllll}h & 2 & h & \ddots\end{array}\right)_{\eta_{8 S}}$ & $\left(\begin{array}{llll}(h & 2 & h & \ddots\end{array}\right)_{\eta_{113}}$ \\
\hline$(\overline{2 h} h \quad 0)_{\eta_{2 B}}$ & $(h \overline{2 h} \odot)_{\eta \mathrm{BL}}$ & $\left(\begin{array}{llll}\hbar & 2 & h & 0\end{array}\right)_{\eta_{B R}}$ & $\left(\begin{array}{llll}h & 2 h & \ddots\end{array}\right)_{\eta_{11 L}}$ \\
\hline$(2 h h \theta)_{\eta_{2 c}}$ & $(h 2 \hbar \theta)_{\text {तอ }}$ & $\left(\begin{array}{llll}h & 2 & h & 0\end{array}\right)_{\eta_{88}}$ & $(h 2 h \theta)_{\eta_{118}}$ \\
\hline$(\overline{2 h} h \theta)_{\eta_{20}}$ & $(h \overline{2 h} 0)_{\eta_{\mathrm{BP}}}$ & $\left(\begin{array}{llll}\hbar & 2 & h & \ddots\end{array}\right){ }_{7 \mathrm{BC}}$ & $(h \overline{2 h} \theta)_{\eta_{11 c}}$ \\
\hline
\end{tabular}

\begin{tabular}{|c|c|c|}
\hline \multicolumn{3}{|c|}{$h \quad h \quad \Theta$}$\quad 3$-fold overlap \\
\hline$(h 2 h \quad 0)_{\eta_{1 A}}$ & $\left(\begin{array}{llll}h & 2 h & 0\end{array}\right)_{\eta_{30}}$ & $(\hbar \hbar \odot)_{\eta_{3 \mathrm{~B}}}$ \\
\hline$(h \overline{2 h} 0)_{\eta_{1 \mathrm{~B}}}$ & $\left(\begin{array}{llll}h & h & 0\end{array}\right)_{\eta_{3 A}}$ & $\left(\hbar \begin{array}{llll}\hbar & 2 & h & 0\end{array}\right)_{\eta_{30}}$ \\
\hline$(h \overline{2 h} \theta)_{\eta_{1 c}}$ & $(\overline{2 h} h \quad 0)_{3 A}$ & $(2 h \hbar \theta)_{\eta_{38}}$ \\
\hline$(h \overline{2 h} 0)_{\eta_{10}}$ & $(2 h h \odot)_{\eta_{3 c}}$ & $(2 h \bar{h} \theta)_{\eta_{30}}$ \\
\hline$(h 2 h \quad 0)_{\eta_{1 E}}$ & $\left(\begin{array}{lll}h & h & \Theta\end{array}\right)_{n_{3 c}}$ & $\left(\begin{array}{llll}h & 2 & h & 0\end{array}\right)_{\eta_{38}}$ \\
\hline$(h \overline{2 h} \theta)_{\eta_{1 F}}$ & $\left(\begin{array}{lll}h & h & 0\end{array}\right)_{\eta_{30}}$ & $\left(\begin{array}{llll}h & 2 & h & 0\end{array}\right)_{\eta_{3 A}}$ \\
\hline
\end{tabular}


$\left\{\begin{array}{lll}0 & 0\end{array}\right\}$

\begin{tabular}{|c|c|c|c|c|c|}
\hline \multicolumn{6}{|c|}{$\left.\begin{array}{llll}0 & 0 & 1\end{array}\right\}$ 6-fold overlap } \\
\hline$\left(\begin{array}{llll}0 & 0 & 1\end{array}\right)_{\eta_{1 \mathrm{~A}}}$ & $\left(\begin{array}{lll}0 & 0 & 1\end{array}\right)_{\eta_{4 A}}$ & $\left(\begin{array}{llll}( & \odot & I\end{array}\right)_{\eta_{48}}$ & $\left(\begin{array}{lll}0 & 0 & 1\end{array}\right)_{\eta_{9 A}}$ & $\left(\begin{array}{llll}0 & 0 & 1\end{array}\right)_{\eta_{11 \mathrm{~A}}}$ & $(\odot \odot \odot I)_{\eta_{11 \mathrm{~B}}}$ \\
\hline$\left(\begin{array}{llll}\odot & 0 & 1\end{array}\right)_{\eta_{1 \mathrm{~B}}}$ & $\left(\begin{array}{llll}0 & 0 & 1\end{array}\right)_{n_{4 C}}$ & $\left(\begin{array}{llll}0 & \odot & I\end{array}\right)_{\eta_{A D}}$ & $\left(\begin{array}{llll}0 & \odot & 1\end{array}\right)_{\eta_{9 \mathrm{~B}}}$ & $\left(\begin{array}{llll}0 & 0 & 1\end{array}\right)_{\eta_{11 c}}$ & $\left(\begin{array}{llll}\odot & \odot & I\end{array}\right)_{n_{110}}$ \\
\hline$\left(\begin{array}{lll}0 & \odot & 1\end{array}\right)_{\eta_{1 c}}$ & $\left(\begin{array}{llll}0 & \odot & 1\end{array}\right)_{\eta_{4 E}}$ & $\left(\begin{array}{lll}0 & \odot & I\end{array}\right)_{\eta_{A F}}$ & $\left(\begin{array}{lll}0 & \odot & 1\end{array}\right)_{\eta_{9 c}}$ & $\left(\begin{array}{llll}\odot & \odot & 1\end{array}\right)_{\eta_{11 \mathrm{E}}}$ & $\left(\begin{array}{llll}\odot & \odot & I\end{array}\right)_{\eta_{11 F}}$ \\
\hline$\left(\begin{array}{lll}0 & 0 & 1\end{array}\right)_{\eta_{10}}$ & $\left(\begin{array}{lll}0 & 0 & 1\end{array}\right)_{r_{46}}$ & $\left(\begin{array}{lll}\odot & \odot & I\end{array}\right)_{\eta_{\text {AH }}}$ & $\left(\begin{array}{lll}0 & 0 & 1\end{array}\right)_{\eta_{90}}$ & $(\odot \odot 1)_{r_{116}}$ & $(\odot \odot)_{\eta_{11 \mathrm{H}}}$ \\
\hline$\left(\begin{array}{llll}0 & \odot & 1\end{array}\right)_{\eta_{1 E}}$ & $\left(\begin{array}{llll}\odot & \odot & 1\end{array}\right)_{\eta_{4 I}}$ & $\left(\begin{array}{llll}0 & \odot & I\end{array}\right)_{\eta_{4 j}}$ & $\left(\begin{array}{llll}0 & 0 & 1\end{array}\right)_{\eta_{9 E}}$ & $\left(\begin{array}{llll}0 & 0 & 1\end{array}\right)_{\eta_{111}}$ & $\left(\begin{array}{llll}\odot & \odot & I\end{array}\right)_{\eta_{11 J}}$ \\
\hline$\left(\begin{array}{llll}0 & \odot & 1\end{array}\right)_{\eta_{1 F}}$ & $\left(\begin{array}{llll}\odot & \odot & 1\end{array}\right)_{\eta_{4 k}}$ & $\left(\begin{array}{llll}0 & \odot & I\end{array}\right)_{\eta_{4 L}}$ & $\left(\begin{array}{lll}0 & \odot & 1\end{array}\right)_{\eta_{9 F}}$ & $\left(\begin{array}{lll}\odot & \odot & 1\end{array}\right)_{\eta_{11 k}}$ & $\left(\begin{array}{lll}\odot & \odot & I\end{array}\right)_{\eta_{11 L}}$ \\
\hline \multicolumn{4}{|c|}{$\begin{array}{llll}0 & 0 & 1\end{array}$}$\quad$ 4-fold overlap & & \\
\hline$\left(\begin{array}{llll}0 & 0 & 1\end{array}\right)_{n_{2 A}}$ & $\left(\begin{array}{llll}0 & 0 & 1\end{array}\right)_{\eta_{3 A}}$ & $\left(\begin{array}{llll}\odot & \odot & 1\end{array}\right)_{\eta_{10 A}}$ & $\left(\begin{array}{llll}\odot & \odot & I\end{array}\right)_{\eta_{106}}$ & & \\
\hline$\left(\begin{array}{lll}0 & 0 & 1\end{array}\right)_{\eta_{2 B}}$ & $\left(\begin{array}{llll}0 & 0 & 1\end{array}\right)_{\eta_{38}}$ & $\left(\begin{array}{llll}( & \odot & 1\end{array}\right)_{\eta_{100}}$ & $\left(\begin{array}{llll}(\odot & \odot & I\end{array}\right)_{\eta_{10 F}}$ & & \\
\hline$\left(\begin{array}{lll}\odot & \odot & 1\end{array}\right)_{\eta_{2 C}}$ & $\left(\begin{array}{llll}0 & \odot & 1\end{array}\right)_{\eta_{3 c}}$ & $(\odot \quad \odot 1)_{\eta_{10 E}}$ & $(\odot \quad \odot \quad I)_{\eta_{108}}$ & & \\
\hline$\left(\begin{array}{lll}0 & \odot & 1\end{array}\right)_{\eta_{20}}$ & $\left(\begin{array}{llll}\odot & \odot & 1\end{array}\right)_{\eta_{30}}$ & $\left(\begin{array}{llll}\odot & \odot & 1\end{array}\right)_{\eta_{1 \mathrm{HH}}}$ & $\left(\begin{array}{llll}\odot & \odot & I\end{array}\right)_{\eta_{10 c}}$ & & \\
\hline
\end{tabular}

$\{h \quad 00\}$

\begin{tabular}{|c|c|c|}
\hline \multicolumn{3}{|c|}{$\begin{array}{llll}h & 0 & 0\end{array}$} 3-fold overlap \\
\hline$\left(\begin{array}{llll}h & 0 & 0\end{array}\right)_{\eta_{2 A}}$ & $\left(\begin{array}{lll}0 & h & 0\end{array}\right)_{\eta_{2 C}}$ & $\left(\begin{array}{llll}h & \odot & 0\end{array}\right)_{\eta_{98}}$ \\
\hline 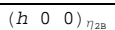 & $\left(\begin{array}{llllll} & h & \Theta\end{array}\right)_{\eta_{20}}$ & 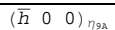 \\
\hline$\left(\begin{array}{llll}h & 0 & 0\end{array}\right)_{\eta_{2 c}}$ & $\left(\begin{array}{llll}0 & h & 0\end{array}\right)_{\eta_{2 B}}$ & $\left(\begin{array}{llll}h & 0 & 0\end{array}\right)_{\eta_{9 E}}$ \\
\hline$\left(\begin{array}{llll}h & 0 & 0\end{array}\right)_{\eta_{20}}$ & $\left(\begin{array}{lll}0 & h & 0\end{array}\right)_{\eta_{2 A}}$ & $\left(\begin{array}{llll}h & \odot & 0\end{array}\right)_{\eta_{9 F}}$ \\
\hline$\left(\begin{array}{lll}(h & h & \ddots\end{array}\right)_{\eta_{2 A}}$ & $(h \bar{h} \Theta)_{\eta_{28}}$ & $\left.\begin{array}{llll}(h & \odot & 0\end{array}\right)_{\eta_{9 C}}$ \\
\hline$\left(\begin{array}{lll}h & h & 0\end{array}\right)_{\eta_{2 c}}$ & 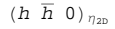 & $\left(\begin{array}{llll}h & \odot & 0\end{array}\right)_{\eta_{90}}$ \\
\hline$(h \circ 00)_{\eta_{11 A}}$ & $(h \odot \odot)_{\eta_{116}}$ & $(h \quad 0 \quad 0)_{\eta_{111}}$ \\
\hline$(h \odot \odot)_{\eta_{118}}$ & $(h \circ 0)_{\eta_{11 E}}$ & $(h \odot \theta 0)_{\eta_{11 k}}$ \\
\hline$\left(\begin{array}{lll}h & 0 & 0\end{array}\right)_{\eta_{11 c}}$ & $\left(\begin{array}{llll}h & 0 & 0\end{array}\right)_{\eta_{11 F}}$ & $\left(\begin{array}{llll}h & 0 & 0\end{array}\right)_{\eta_{11 I}}$ \\
\hline$\left(\begin{array}{llll}h & 0 & 0\end{array}\right)_{\eta_{110}}$ & $(h \quad \odot \quad 0)_{\eta 11 H}$ & $\left(\begin{array}{llll}h & \odot & 0\end{array}\right)_{r_{11 L}}$ \\
\hline
\end{tabular}

